\title{
Traceless Staudinger ligation of glycosyl azides with triaryl phosphines: steroselective synthesis of glycosyl amides
}

\author{
Aldo Bianchi, Anna Bernardi \\ Universita' degli Studi di Milano, Dipartimento di Chimica Organica e Industriale and Centro di \\ Eccellenza CISI, via Venezian 21, 20133 Milano, Italy
}

\begin{abstract}
${ }^{1} \mathrm{H}-,{ }^{13} \mathrm{C}-,{ }^{31} \mathrm{P}-\mathrm{NMR}$ and COSY spectra for compounds: 4a-4i, 11f-11i, 12c, 18a, 19a, 20a, 19b, 19g, 19h, 21g,13g, 14g and 16f.
\end{abstract}

\section{Table of contents}

${ }^{1}$ H-NMR spectrum of diphenylphosphanyl-phenyl acetate 4a $\quad$ S5

${ }^{13} \mathrm{C}$-NMR spectrum of diphenylphosphanyl-phenyl acetate 4a $\quad$ S5

${ }^{31} \mathrm{P}-\mathrm{NMR}$ spectrum of diphenylphosphanyl-phenyl acetate 4a $\quad$ S6

${ }^{1}$ H-NMR spectrum of diphenylphosphanyl-phenyl pentanoate $4 \mathbf{b} \quad$ S6

${ }^{13} \mathrm{C}-\mathrm{NMR}$ spectrum of diphenylphosphanyl-phenyl pentanoate $\mathbf{4 b} \quad$ S7

${ }^{31} \mathrm{P}-\mathrm{NMR}$ spectrum of diphenylphosphanyl-phenyl pentanoate $\mathbf{4 b} \quad \mathrm{S} 7$

${ }^{1}$ H-NMR spectrum of diphenylphosphanyl-phenyl 3-methyl-butanoate $4 \mathbf{c} \quad$ S8

${ }^{13} \mathrm{C}$-NMR spectrum of diphenylphosphanyl-phenyl 3-methyl-butanoate $4 \mathbf{c} \quad$ S8

${ }^{31} \mathrm{P}-\mathrm{NMR}$ spectrum of diphenylphosphanyl-phenyl 3-methyl-butanoate $4 \mathbf{c} \quad$ S9

${ }^{1}$ H-NMR spectrum of diphenylphosphanyl-phenyl $i$-butanoate $4 \mathbf{d} \quad$ S9

${ }^{13} \mathrm{C}$-NMR spectrum of diphenylphosphanyl-phenyl $i$-butanoate $\mathbf{4 d} \quad \mathrm{S} 10$

${ }^{31} \mathrm{P}$-NMR spectrum of diphenylphosphanyl-phenyl $i$-butanoate $4 \mathbf{d} \quad \mathrm{S} 10$

${ }^{1} \mathrm{H}-\mathrm{NMR}$ spectrum of diphenylphosphanyl-phenyl 3-methyl-but-2-enoate 4e $\quad$ S11

${ }^{13} \mathrm{C}-\mathrm{NMR}$ spectrum of diphenylphosphanyl-phenyl 3-methyl-but-2-enoate $4 \mathbf{e} \quad \mathrm{S} 11$

${ }^{31} \mathrm{P}-\mathrm{NMR}$ spectrum of diphenylphosphanyl-phenyl 3-methyl-but-2-enoate 4e $\quad \mathrm{S} 12$

${ }^{1}$ H-NMR spectrum of pentanedioic acid 2-diphenylphosphanyl-phenyl ester methyl ester $4 \mathbf{f f} \quad$ S12

${ }^{13} \mathrm{C}-\mathrm{NMR}$ spectrum of pentanedioic acid 2-diphenylphosphanyl-phenyl ester methyl ester $\mathbf{4 f} \quad \mathrm{S} 13$

${ }^{31} \mathrm{P}-\mathrm{NMR}$ spectrum of pentanedioic acid 2-diphenylphosphanyl-phenyl ester methyl ester $\mathbf{4 f} \quad$ S13

${ }^{1} \mathrm{H}-\mathrm{NMR}$ spectrum of $N$-benzyloxycarbonyl-L-aspartic acid 4-(2-diphenylphosphanylphenyl) ester 1-methyl ester $\mathbf{4 g}$

${ }^{31} \mathrm{P}-\mathrm{NMR}$ spectrum of $N$-benzyloxycarbonyl-L-aspartic acid 4-(2-diphenylphosphanylphenyl) ester 1-methyl ester $\mathbf{4 g}$ 
${ }^{1} \mathrm{H}-\mathrm{NMR}$ spectrum of $\mathrm{N}$ - $t$-butoxycarbonyl-L-aspartic acid 4-(2-diphenylphosphanyl-phenyl) ester 1-benzyl ester $\mathbf{4 h}$

${ }^{13} \mathrm{C}$-NMR spectrum of $N$ - $t$-butoxycarbonyl-L-aspartic acid 4-(2-diphenylphosphanylphenyl) ester 1-benzyl ester $\mathbf{4 h}$

${ }^{31} \mathrm{P}-\mathrm{NMR}$ spectrum of $N$ - $t$-butoxycarbonyl-L-aspartic acid 4-(2-diphenylphosphanyl-phenyl) ester 1-benzyl ester $\mathbf{4 h}$

${ }^{1} \mathrm{H}-\mathrm{NMR}$ spectrum of $N$-fluoren-9-ylmethoxycarbonyl-L-aspartic acid 4-(2diphenylphosphanyl-phenyl) ester 1-benzyl ester $\mathbf{4 i}$

${ }^{13} \mathrm{C}-\mathrm{NMR}$ spectrum of $N$-fluoren-9-ylmethoxycarbonyl-L-aspartic acid 4-(2diphenylphosphanyl-phenyl) ester 1-benzyl ester 4i

${ }^{31} \mathrm{P}-\mathrm{NMR}$ spectrum of $N$-fluoren-9-ylmethoxycarbonyl-L-aspartic acid 4-(2diphenylphosphanyl-phenyl) ester 1-benzyl ester 4i

${ }^{1} \mathrm{H}-\mathrm{NMR}$ spectrum of $N$ - pentanedioic acid -2,3,4,6-tetra- $O$-benzyl- $\alpha$-Dglucopyranosylamide methyl ester $\mathbf{1 1 f}$

${ }^{13} \mathrm{C}$-NMR spectrum of $N$ - pentanedioic acid -2,3,4,6-tetra- $O$-benzyl- $\alpha$-Dglucopyranosylamide methyl ester $\mathbf{1 1 f}$

${ }^{1} \mathrm{H}-\mathrm{NMR}$ spectrum of $N^{\alpha}$-benzyloxycarbonyl- $N^{\gamma}$-(2,3,4,6-tetra- $O$-benzyl- $\alpha$-Dglucopyranosyl)-L-asparagine- $O$-methyl ester $\mathbf{1 1 g}$

${ }^{13} \mathrm{C}-\mathrm{NMR}$ spectrum of $N^{\alpha}$-benzyloxycarbonyl- $N^{\gamma}$-(2,3,4,6-tetra- $O$-benzyl- $\alpha$-Dglucopyranosyl)-L-asparagine- $O$-methyl ester $\mathbf{1 1 g}$

COSY spectrum of $N^{\alpha}$-benzyloxycarbonyl- $N^{\prime}$-(2,3,4,6-tetra- $O$-benzyl- $\alpha$-D-glucopyranosyl)L-asparagine- $O$-methyl ester $\mathbf{1 1 g}$

${ }^{1} \mathrm{H}-\mathrm{NMR}$ spectrum of $N^{\alpha}$-t-butoxycarbonyl-N ${ }^{\gamma}$-(2,3,4,6-tetra- $O$-benzyl- $\alpha$-Dglucopyranosyl)-L-asparagine- $O$-benzyl ester $\mathbf{1 1 h}$

${ }^{13} \mathrm{C}$-NMR spectrum of $N^{\alpha}$ - $t$-butoxycarbonyl- $\mathrm{N}^{\gamma}$-(2,3,4,6-tetra- $O$-benzyl- $\alpha$-Dglucopyranosyl)-L-asparagine- $O$-benzyl ester $\mathbf{1 1 h}$

COSY spectrum of $N^{\alpha}$-t-butoxycarbonyl-N $N^{\gamma}-(2,3,4,6$-tetra- $O$-benzyl- $\alpha$-D-glucopyranosyl)L-asparagine- $O$-benzyl ester $\mathbf{1 1 h}$

${ }^{1}$ H-NMR spectrum of $N^{\alpha}$-fluoren-9-ylmethoxycarbonyl- $N^{\gamma}$-(2,3,4,6-tetra- $O$-benzyl- $\alpha$-Dglucopyranosyl)-L-asparagine- $O$-allyl ester $\mathbf{1 1 i}$ 
${ }^{13} \mathrm{C}$-NMR spectrum of $N^{\alpha}$-fluoren-9-ylmethoxycarbonyl- $N^{\gamma}$-(2,3,4,6-tetra- $O$-benzyl- $\alpha$-Dglucopyranosyl)-L-asparagine- $O$-allyl ester $11 \mathbf{i}$

COSY spectrum of $N^{\alpha}$-fluoren-9-ylmethoxycarbonyl- $N^{\gamma}$-(2,3,4,6-tetra- $O$-benzyl- $\alpha$-Dglucopyranosyl)-L-asparagine- $O$-allyl ester 11i

${ }^{1}$ H-NMR spectrum of $N$-(3-methylbutanoyl)-2,3,4,6-tetra- $O$-benzyl- $\alpha$-Dgalactopyranosylamine 12c

${ }^{13} \mathrm{C}-\mathrm{NMR}$ spectrum of $N$-(3-methylbutanoyl)-2,3,4,6-tetra- $O$-benzyl- $\alpha$-Dgalactopyranosylamine 12c

COSY spectrum of $N$-(3-methylbutanoyl)-2,3,4,6-tetra-O-benzyl- $\alpha$-Dgalactopyranosylamine 12c

${ }^{1}$ H-NMR spectrum of $N^{\alpha}$-benzyloxycarbonyl- $N^{\prime}$-(2,3,4,6-tetra- $O$-benzyl- $\alpha / \beta$-Dgalactopyranosyl)-L-asparagine- $O$-methyl ester $\mathbf{1 2 g}$

${ }^{1} \mathrm{H}-\mathrm{NMR}$ spectrum of $N^{\alpha}$-t-butoxycarbonyl- $N^{\gamma}$-(2,3,4,6-tetra- $O$-benzyl- $\alpha / \beta$-Dgalactopyranosyl)-L-asparagine- $O$-benzyl ester $\mathbf{1 2 h}$

${ }^{1} \mathrm{H}-\mathrm{NMR}$ spectrum of $N$-acetyl- $\alpha$-L-fucopyranosylamine 18a $\quad$ S28

${ }^{13} \mathrm{C}$-NMR spectrum of $N$-acetyl- $\alpha$-L-fucopyranosylamine 18a $\quad$ S28

COSY spectrum of $N$-acetyl- $\alpha$-L-fucopyranosylamine 18a $\quad$ S29

${ }^{1}$ H-NMR spectrum of $N$-acetyl- $\alpha$-D-glucopyranosylamine 19a $\quad$ S30

${ }^{13} \mathrm{C}$-NMR spectrum of $N$-acetyl- $\alpha$-D-glucopyranosylamine 19a $\quad$ S30

$\begin{array}{ll}\text { COSY spectrum of } N \text {-acetyl- } \alpha \text {-D-glucopyranosylamine 19a } & \text { S31 }\end{array}$

${ }^{1}$ H-NMR spectrum of $N$-acetyl- $\alpha$-D-galactopyranosylamine 20a $\quad$ S32

${ }^{13} \mathrm{C}$-NMR spectrum of $N$-acetyl- $\alpha$-D-galactopyranosylamine 20a $\quad$ S32

COSY spectrum of $N$-acetyl- $\alpha$-D-galactopyranosylamine 20a $\quad$ S33

${ }^{1}$ H-NMR spectrum of $N$-pentanoyl- $\alpha$-D-glucopyranosylamine 19b $\quad$ S34

${ }^{13} \mathrm{C}$-NMR spectrum of $N$-pentanoyl- $\alpha$-D-glucopyranosylamine 19b $\quad$ S34

COSY spectrum of $N$-pentanoyl- $\alpha$-D-glucopyranosylamine 19b $\quad$ S35

${ }^{1} \mathrm{H}-\mathrm{NMR}$ spectrum of $N^{\gamma}$-( $\alpha$-D-glucopyranosyl)-L-asparagine- $O$-methyl ester 19g $\quad$ S36

${ }^{13} \mathrm{C}$-NMR spectrum of $N^{\gamma}$-( $\alpha$-D-glucopyranosyl)-L-asparagine- $O$-methyl ester $19 \mathrm{~g} \quad \mathrm{~S} 36$

COSY spectrum of $N^{\prime}$-( $\alpha$-D-glucopyranosyl)-L-asparagine- $O$-methyl ester 19g $\quad$ S37

${ }^{1}$ H-NMR spectrum of $N^{\alpha}$-t-butoxycarbonyl- $N^{\gamma}$-( $\alpha$-D-glucopyranosyl)-L-asparagine 19h $\quad$ S38

${ }^{13} \mathrm{C}$-NMR spectrum of $N^{\alpha}$-t-butoxycarbonyl- $N^{\prime}-(\alpha$-D-glucopyranosyl)-L-asparagine $19 \mathbf{h} \quad$ S38 
COSY spectrum of $N^{\alpha}$-t-butoxycarbonyl- $N^{\prime}$-( $\alpha$-D-glucopyranosyl)-L-asparagine 19h

${ }^{1}$ H-NMR spectrum of $N^{\alpha}$-acetyl- $N^{\gamma}$-(2,3,4,6-tetra- $O$-acetyl- $\alpha$-D-glucopyranosyl)-Lasparagine- $O$-methyl ester $\mathbf{2 1 g}$

${ }^{13} \mathrm{C}$-NMR spectrum of $N^{\alpha}$-acetyl- $N^{\prime}$-(2,3,4,6-tetra- $O$-acetyl- $\alpha$-D-glucopyranosyl)-Lasparagine- $O$-methyl ester $\mathbf{2 1 g}$

COSY spectrum of $N^{\alpha}$-acetyl- $N^{\prime}$-(2,3,4,6-tetra- $O$-acetyl- $\alpha$-D-glucopyranosyl)-L-asparagine$O$-methyl ester $\mathbf{2 1 g}$

${ }^{1} \mathrm{H}$-NMR spectrum of $N^{\alpha}$-carbobenzyloxy- $N^{\gamma}$-(2,3,4,6-tetra- $O$-acetyl- $\beta$-D-glucopyranosyl)L-asparagine- $O$-methyl ester $\mathbf{1 3 g}$

${ }^{13} \mathrm{C}$-NMR spectrum of $N^{\alpha}$-carbobenzyloxy- $N^{\gamma}$-(2,3,4,6-tetra- $O$-acetyl- $\beta$-D-glucopyranosyl)L-asparagine- $O$-methyl ester $\mathbf{1 3 g}$

COSY spectrum of $N^{\alpha}$-carbobenzyloxy- $N^{\prime}$-(2,3,4,6-tetra- $O$-acetyl- $\beta$-D-glucopyranosyl)-Lasparagine- $O$-methyl ester $\mathbf{1 3 g}$

${ }^{1} \mathrm{H}-\mathrm{NMR}$ spectrum of $N^{\alpha}$-carbonzyloxy- $N^{\gamma}$-(3,4,6-tri- $O$-acetyl-2- $N$-acetyl-2-deoxy- $\beta$-Dglucopyranosyl)-L-asparagine- $O$-methyl ester $\mathbf{1 4 g}$

${ }^{13} \mathrm{C}$-NMR spectrum of $N^{\alpha}$-carbonzyloxy- $N^{\gamma}$-(3,4,6-tri- $O$-acetyl-2- $N$-acetyl-2-deoxy- $\beta$-Dglucopyranosyl)-L-asparagine- $O$-methyl ester $\mathbf{1 4 g}$

COSY spectrum of $N^{\alpha}$-carbonzyloxy- $N^{\gamma}$-(3,4,6-tri- $O$-acetyl-2- $N$-acetyl-2-deoxy- $\beta$-Dglucopyranosyl)-L-asparagine- $O$-methyl ester $\mathbf{1 4 g}$

${ }^{1} \mathrm{H}-\mathrm{NMR}$ spectrum of 1- $\mathrm{N}$ - pentanedioic acid -2- $N$-phthalimido-2-deoxy-3,4,6-tri- $O$-acetyl$\beta$-D-glucopyranosyl amide methyl ester $\mathbf{1 6 f}$

${ }^{13} \mathrm{C}$-NMR spectrum of $1-N$ - pentanedioic acid -2- $N$-phthalimido-2-deoxy-3,4,6-tri- $O$-acetyl$\beta$-D-glucopyranosyl amide methyl ester $\mathbf{1 6 f}$

COSY spectrum of 1- $N$ - pentanedioic acid -2- $N$-phthalimido-2-deoxy-3,4,6-tri- $O$-acetyl- $\beta$ D-glucopyranosyl amide methyl ester $\mathbf{1 6 f}$ 
Diphenylphosphanyl-phenyl acetate (4a)
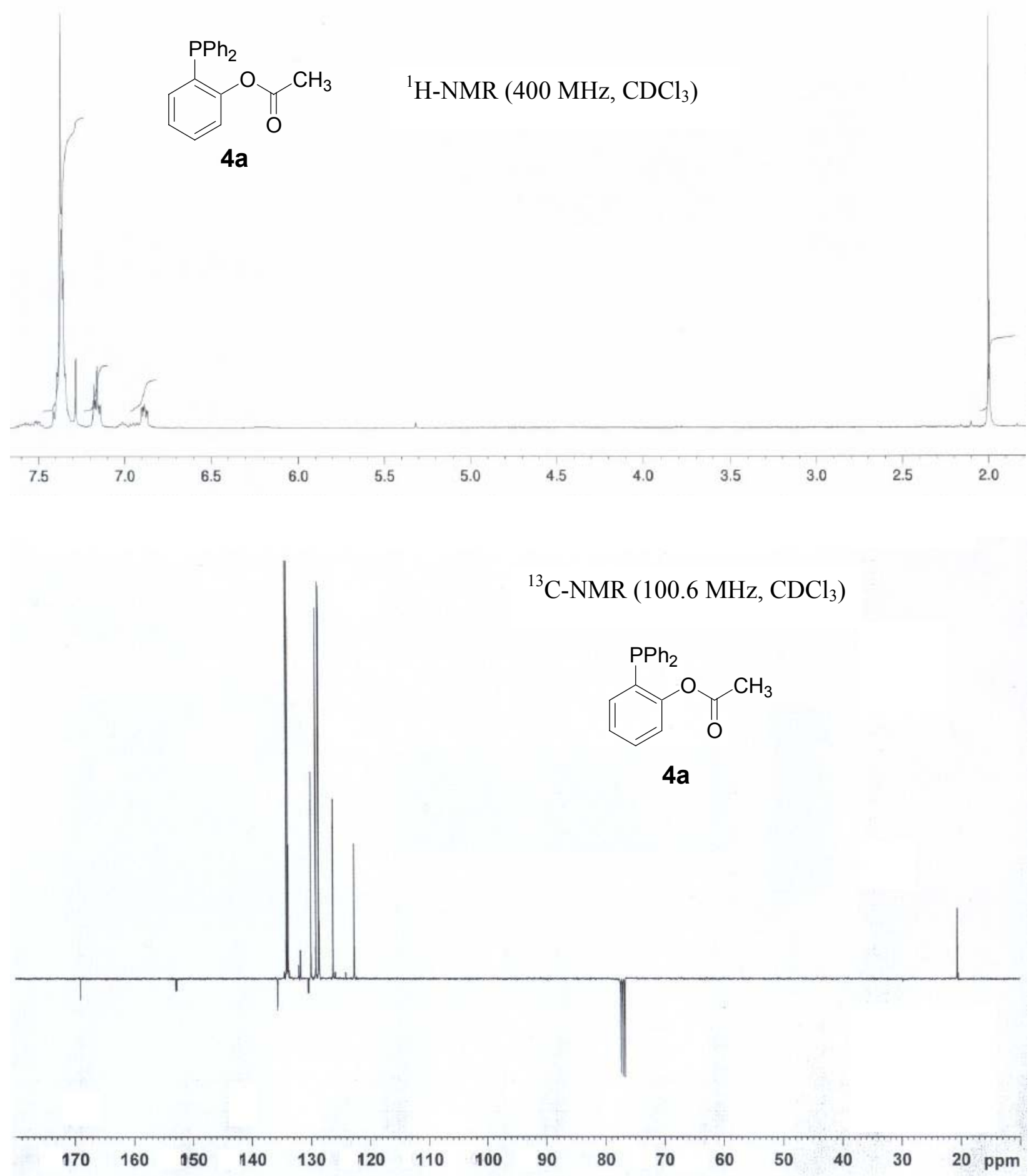


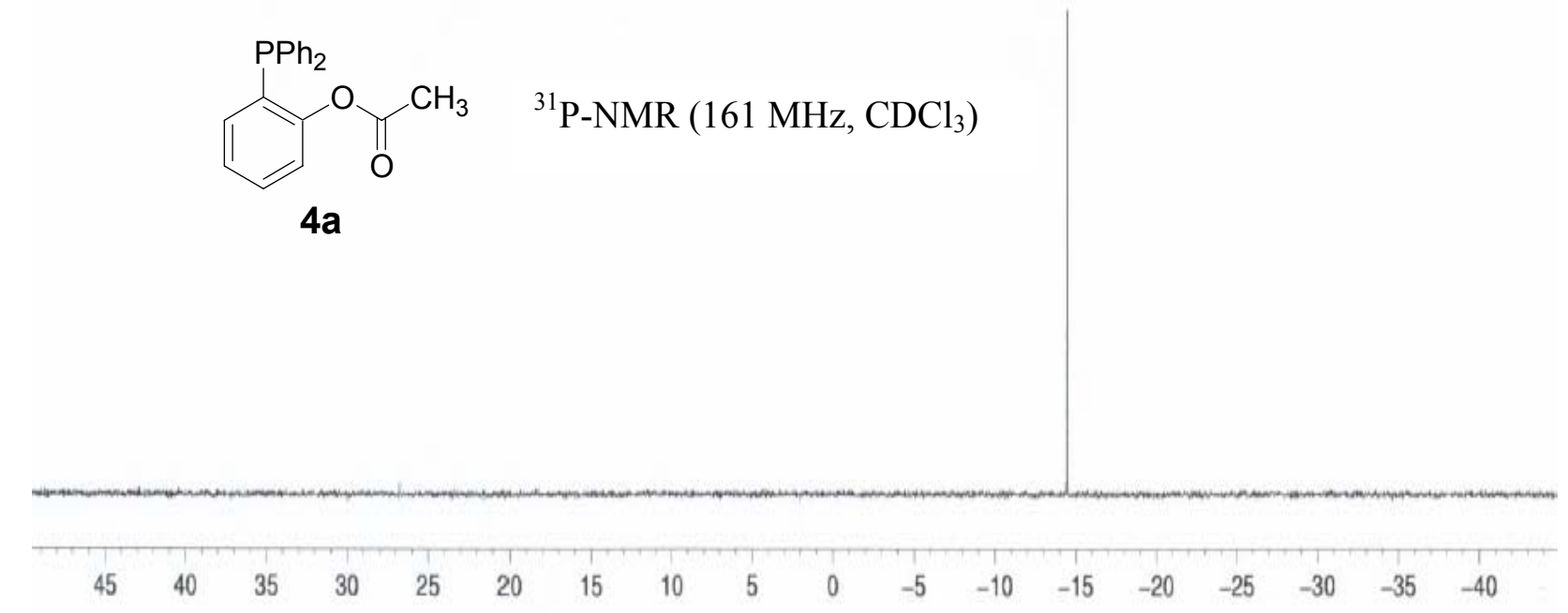

Diphenylphosphanyl-phenyl pentanoate (4b)

${ }^{1} \mathrm{H}-\mathrm{NMR}\left(400 \mathrm{MHz}, \mathrm{CDCl}_{3}\right)$

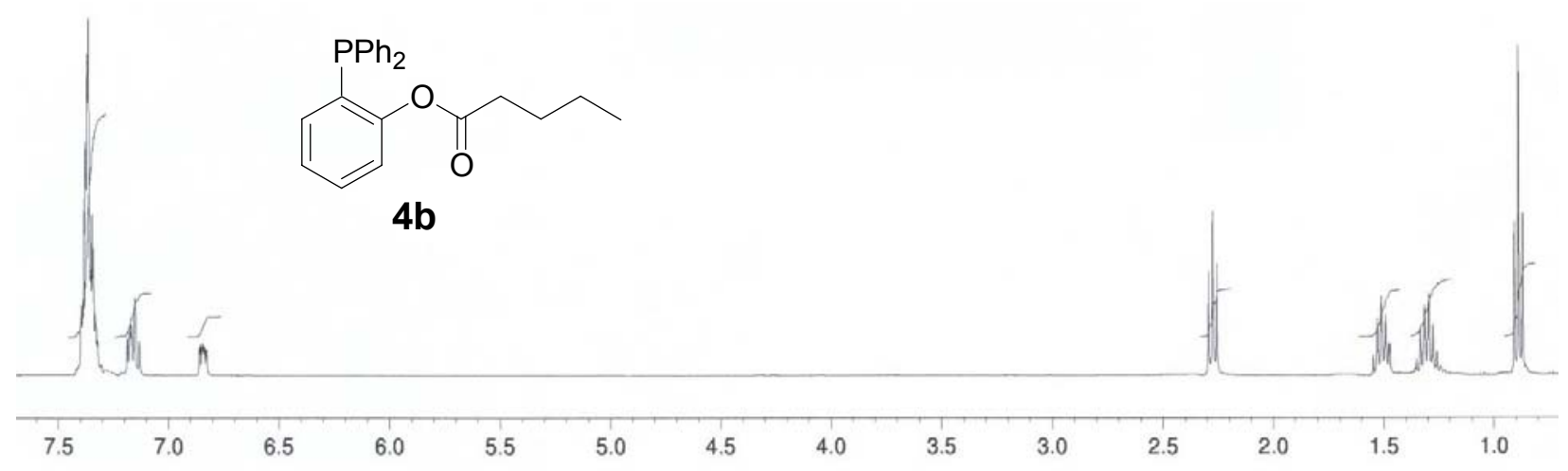



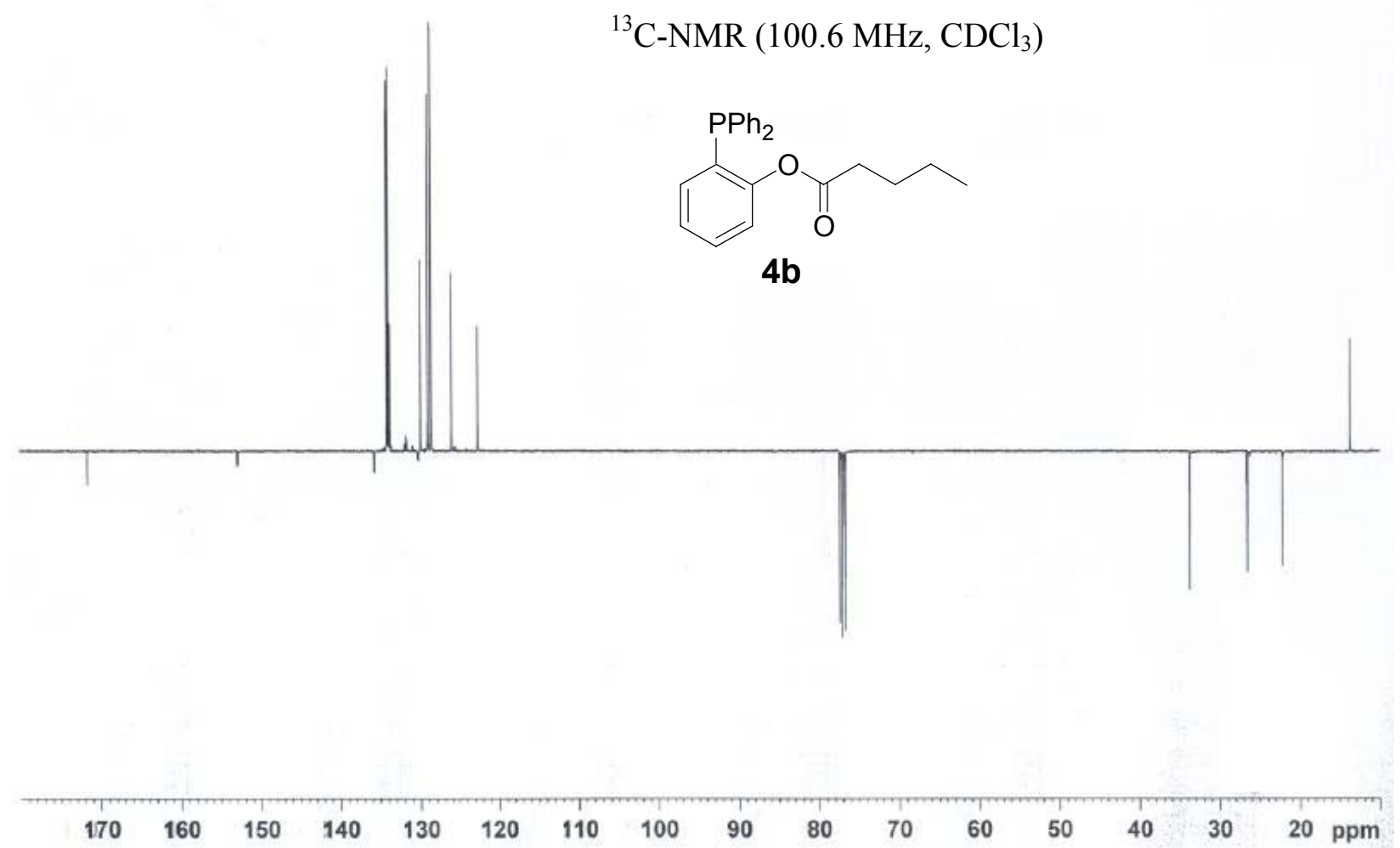

${ }^{31} \mathrm{P}-\mathrm{NMR}\left(161 \mathrm{MHz}, \mathrm{CDCl}_{3}\right.$ )<smiles>CCCCC(=O)Oc1ccccc1-c1ccccc1</smiles>

$4 b$ 
Diphenylphosphanyl-phenyl 3-methyl-butanoate (4c)

${ }^{1} \mathrm{H}-\mathrm{NMR}\left(400 \mathrm{MHz}, \mathrm{CDCl}_{3}\right.$ )
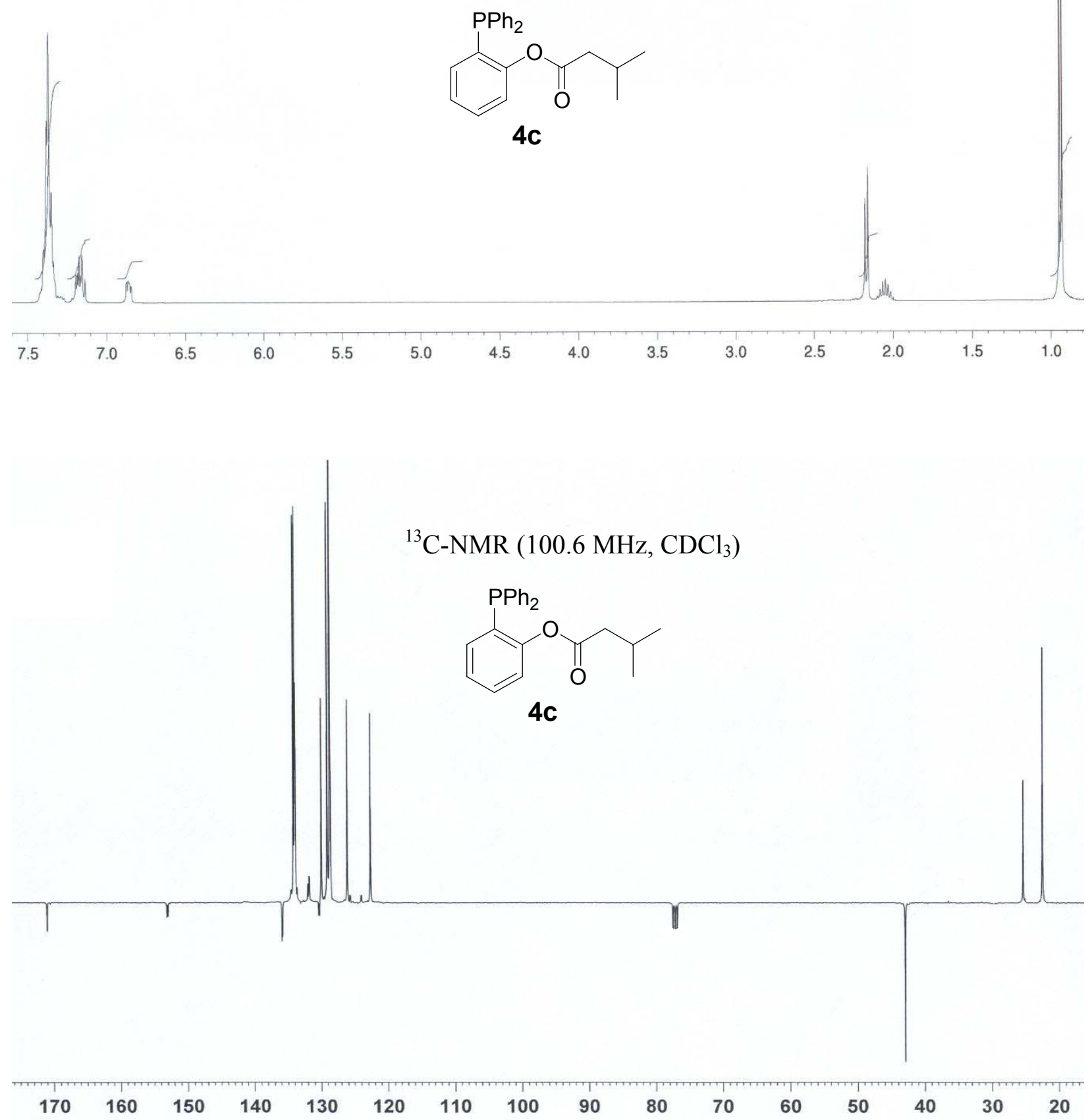


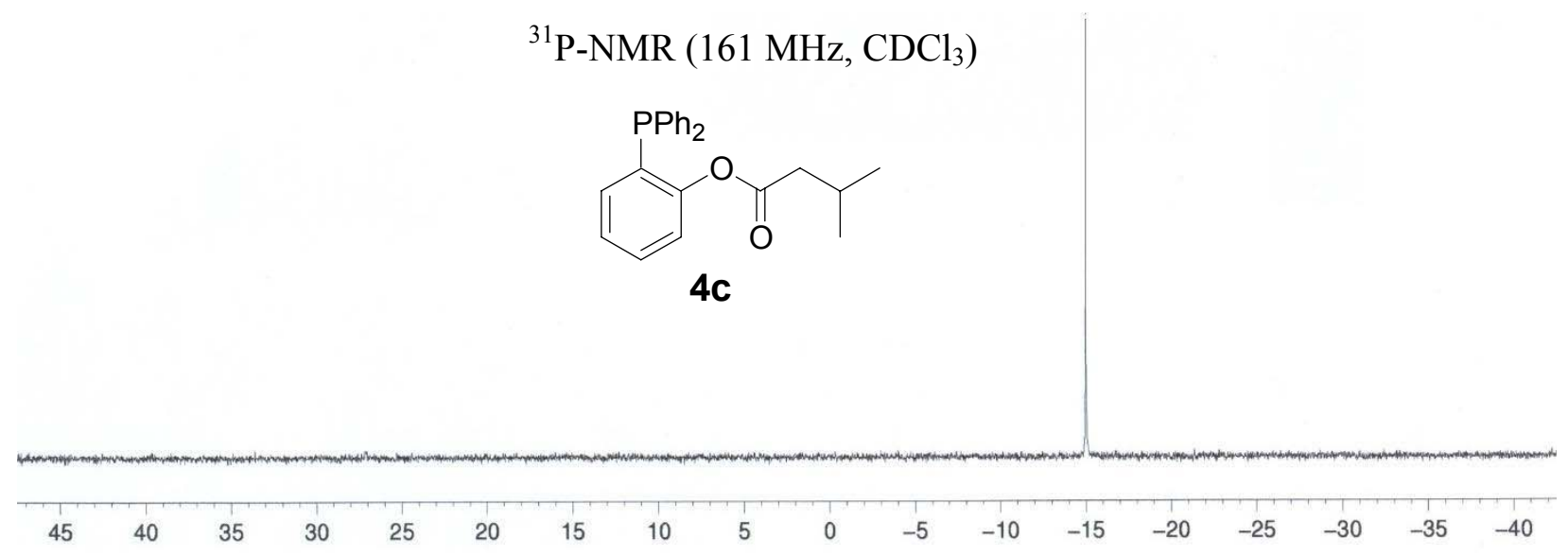

Diphenylphosphanyl-phenyl $i$-butanoate (4d)

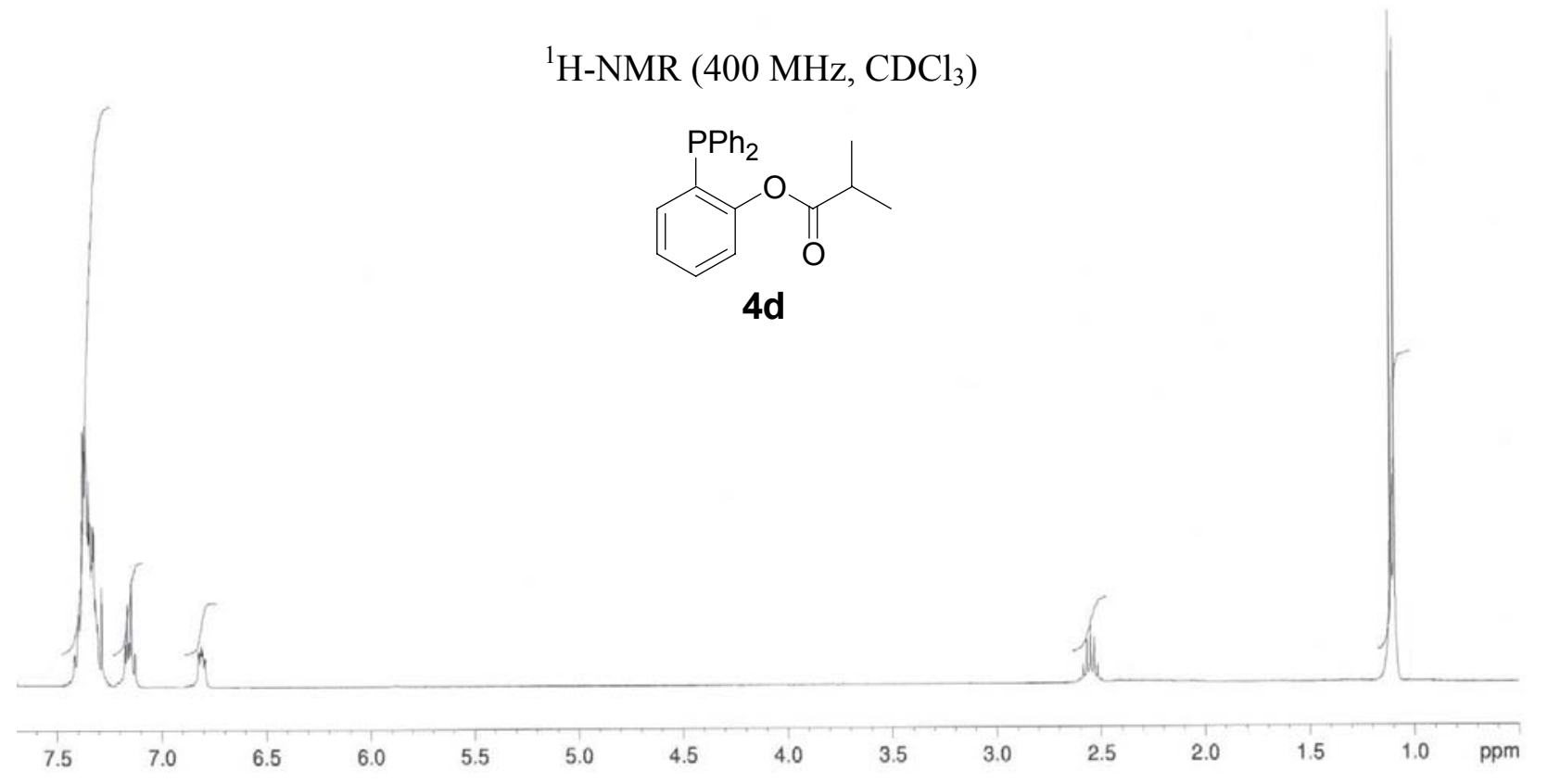




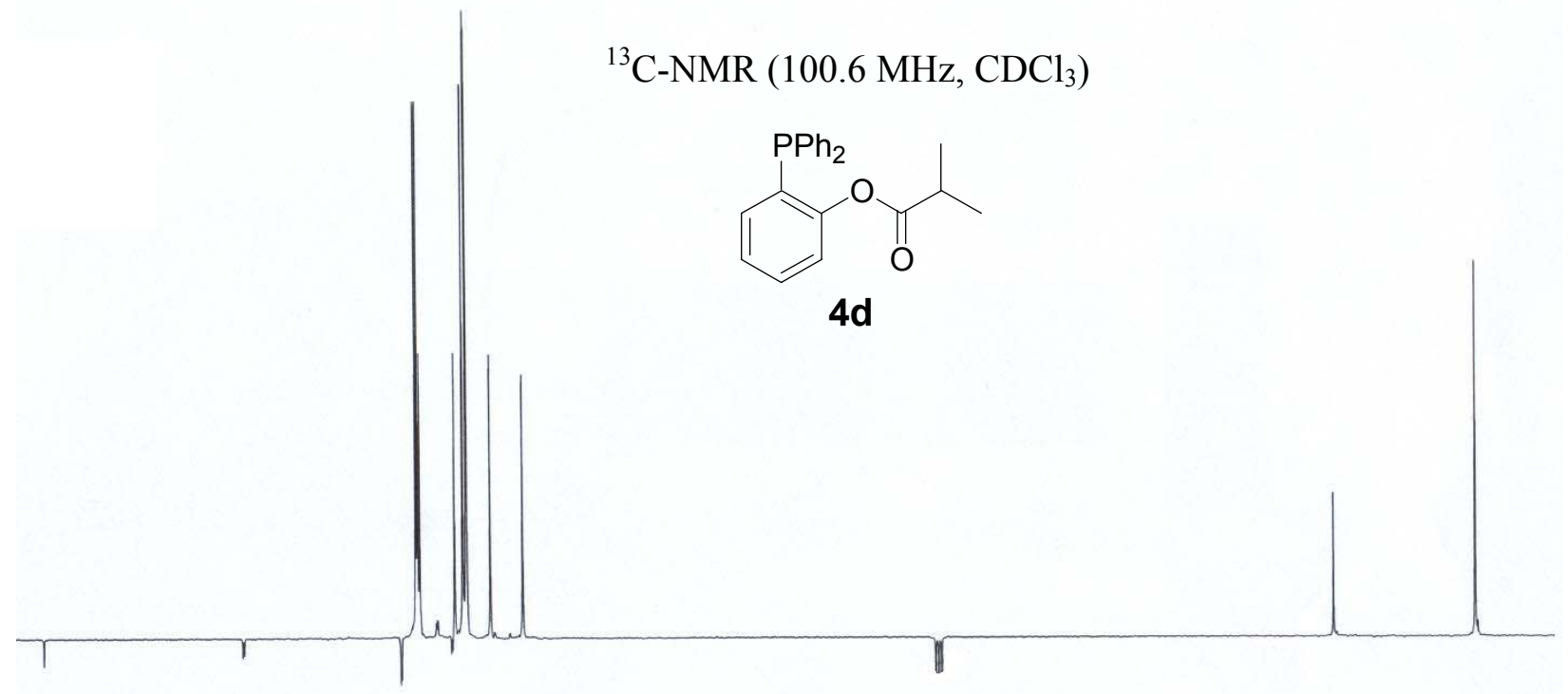

\begin{tabular}{|c|c|c|c|c|c|c|c|c|c|c|c|c|c|c|c|}
\hline 170 & 160 & 150 & 140 & 130 & 120 & 110 & 100 & 90 & 80 & 70 & 60 & 50 & 40 & 30 & 20 \\
\hline
\end{tabular}

${ }^{31} \mathrm{P}-\mathrm{NMR}\left(161 \mathrm{MHz}, \mathrm{CDCl}_{3}\right)$

$\underbrace{\mathrm{P}_{\mathrm{d}}}_{4 \mathbf{d}}$

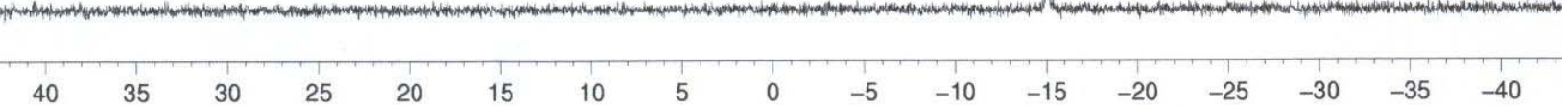


Diphenylphosphanyl-phenyl 3-methyl-but-2-enoate (4e)
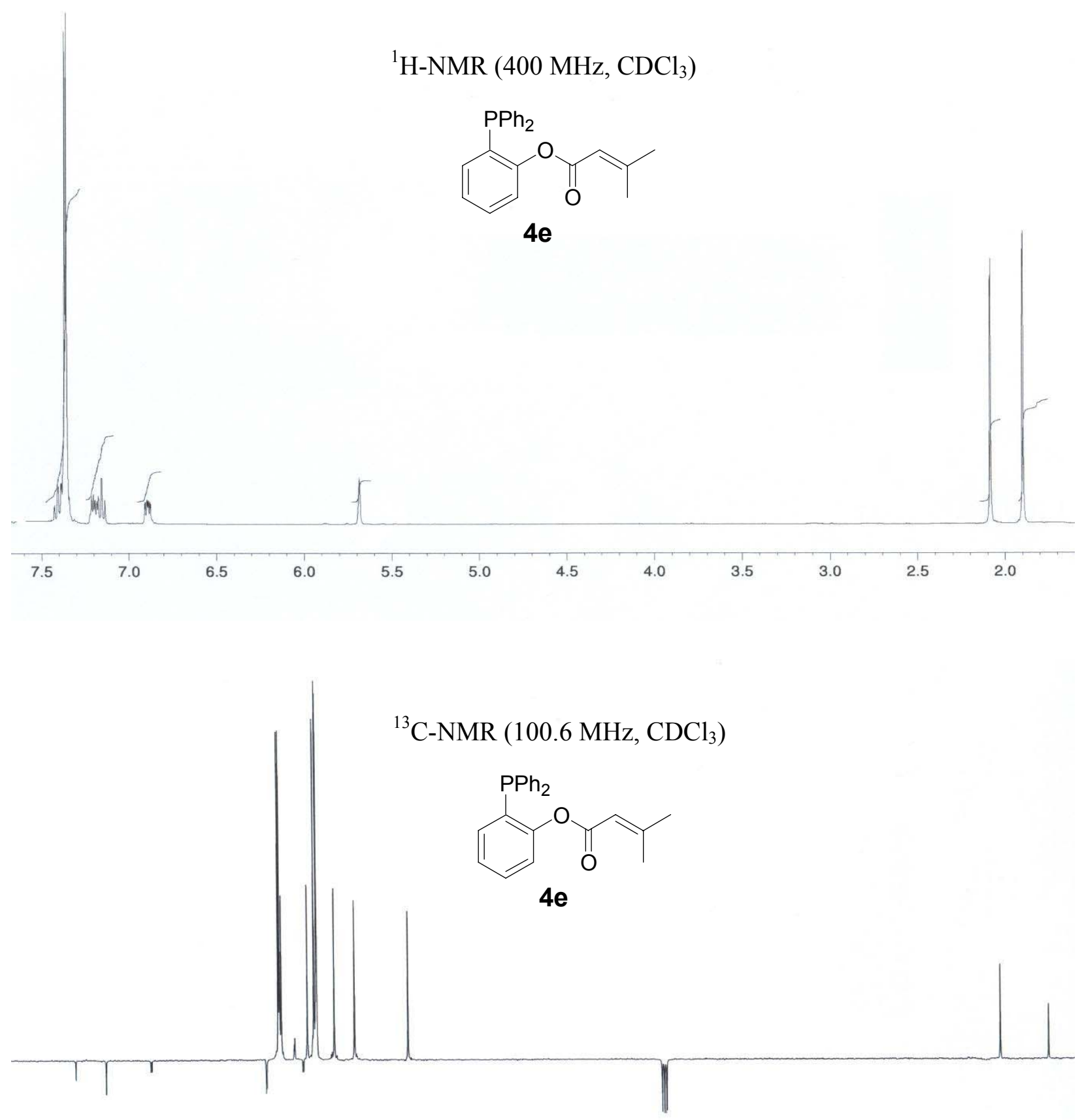

$\begin{array}{llllllllllllllll}170 & 160 & 150 & 140 & 130 & 120 & 110 & 100 & 90 & 80 & 70 & 60 & 50 & 40 & 30 & 20\end{array}$


${ }^{31} \mathrm{P}-\mathrm{NMR}\left(161 \mathrm{MHz}, \mathrm{CDCl}_{3}\right)$<smiles>CC(C)=CC(=O)Oc1ccccc1-c1ccccc1</smiles>

$\begin{array}{llllllllllllllllll}40 & 35 & 30 & 25 & 20 & 15 & 10 & 5 & 0 & -5 & -10 & -15 & -20 & -25 & -30 & -35 & -40\end{array}$

Pentanedioic acid 2-diphenylphosphanyl-phenyl ester methyl ester (4f)

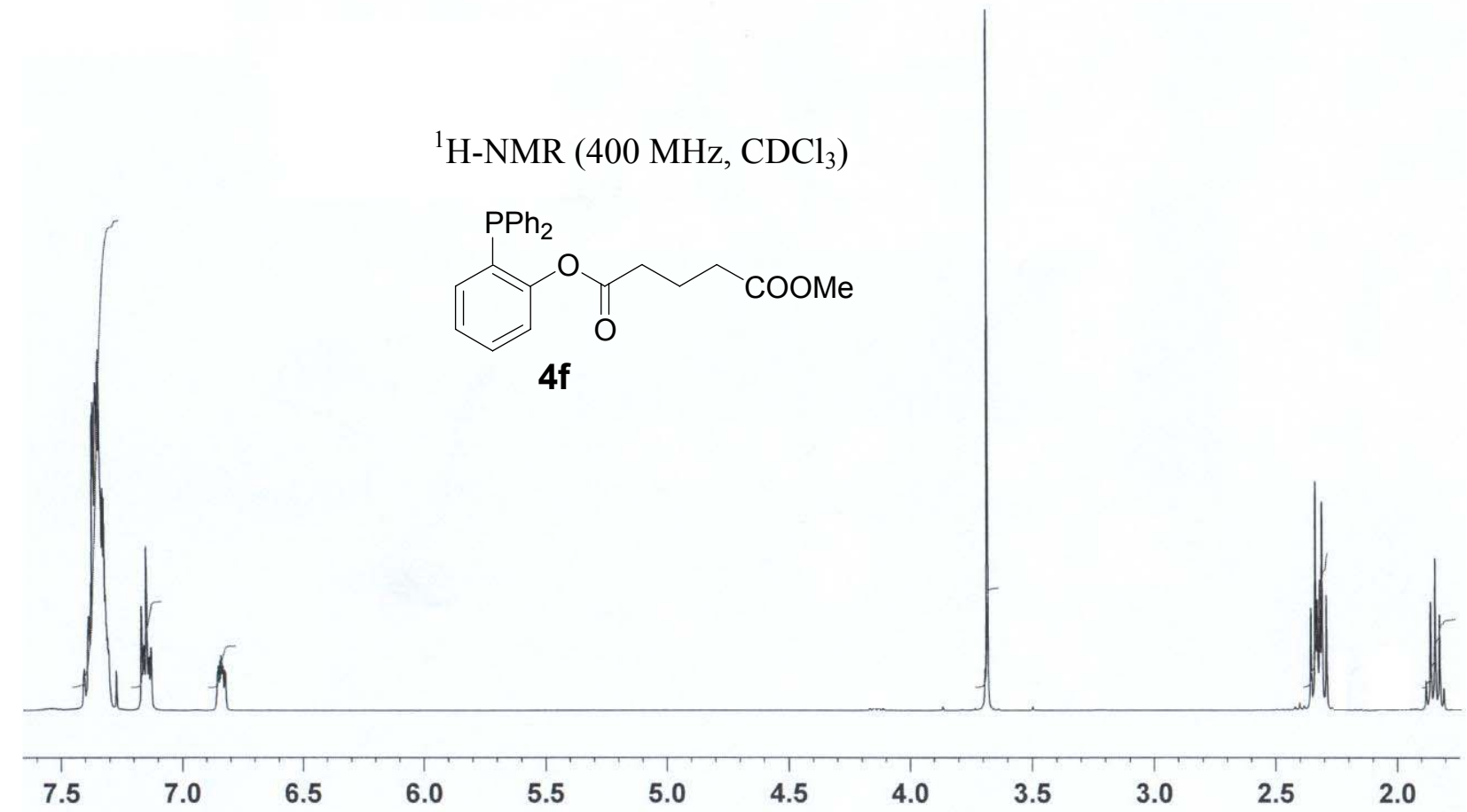



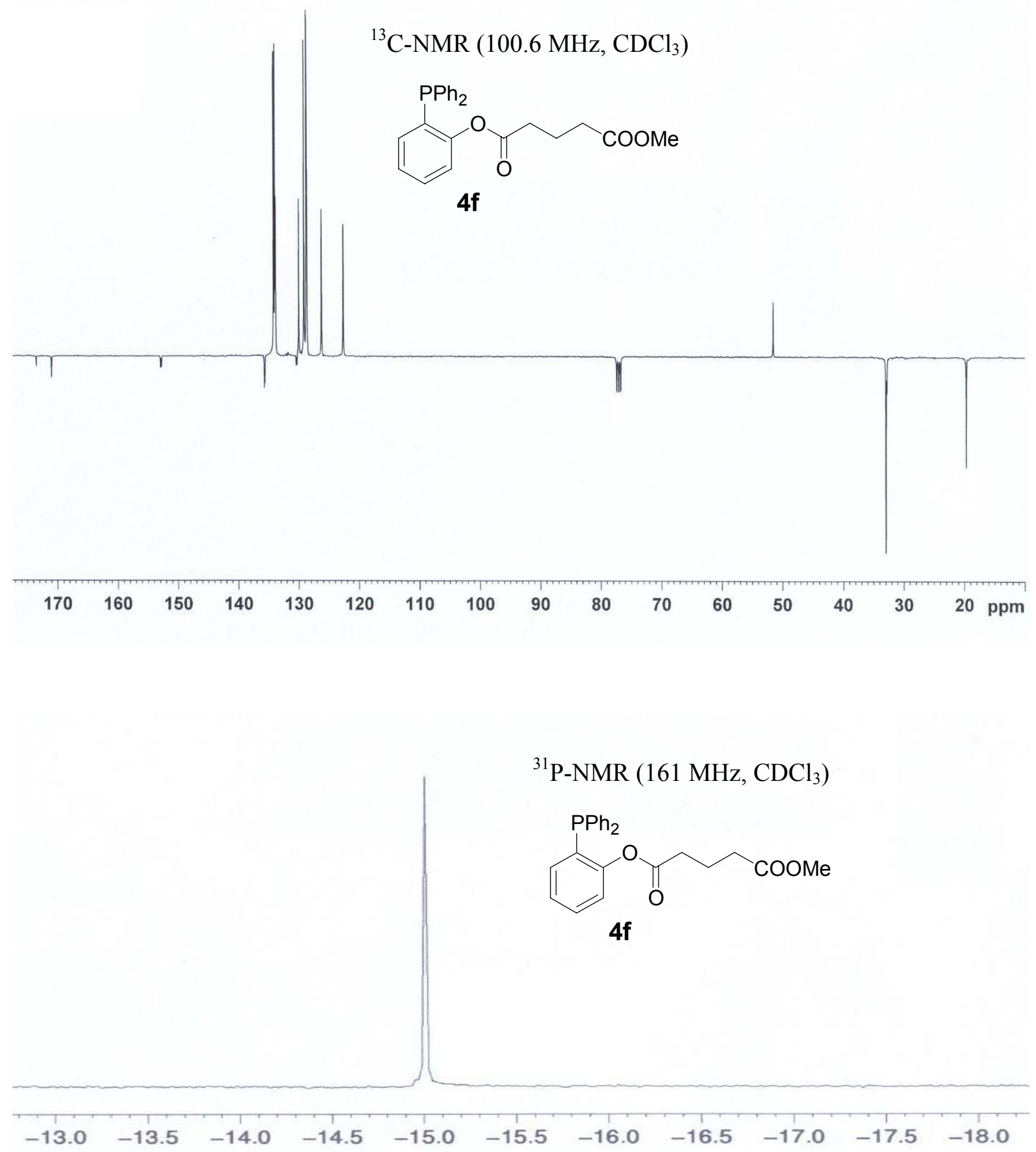
$N$-Benzyloxycarbonyl-L-aspartic acid 4-(2-diphenylphosphanyl-phenyl) ester 1-methyl ester (4g)

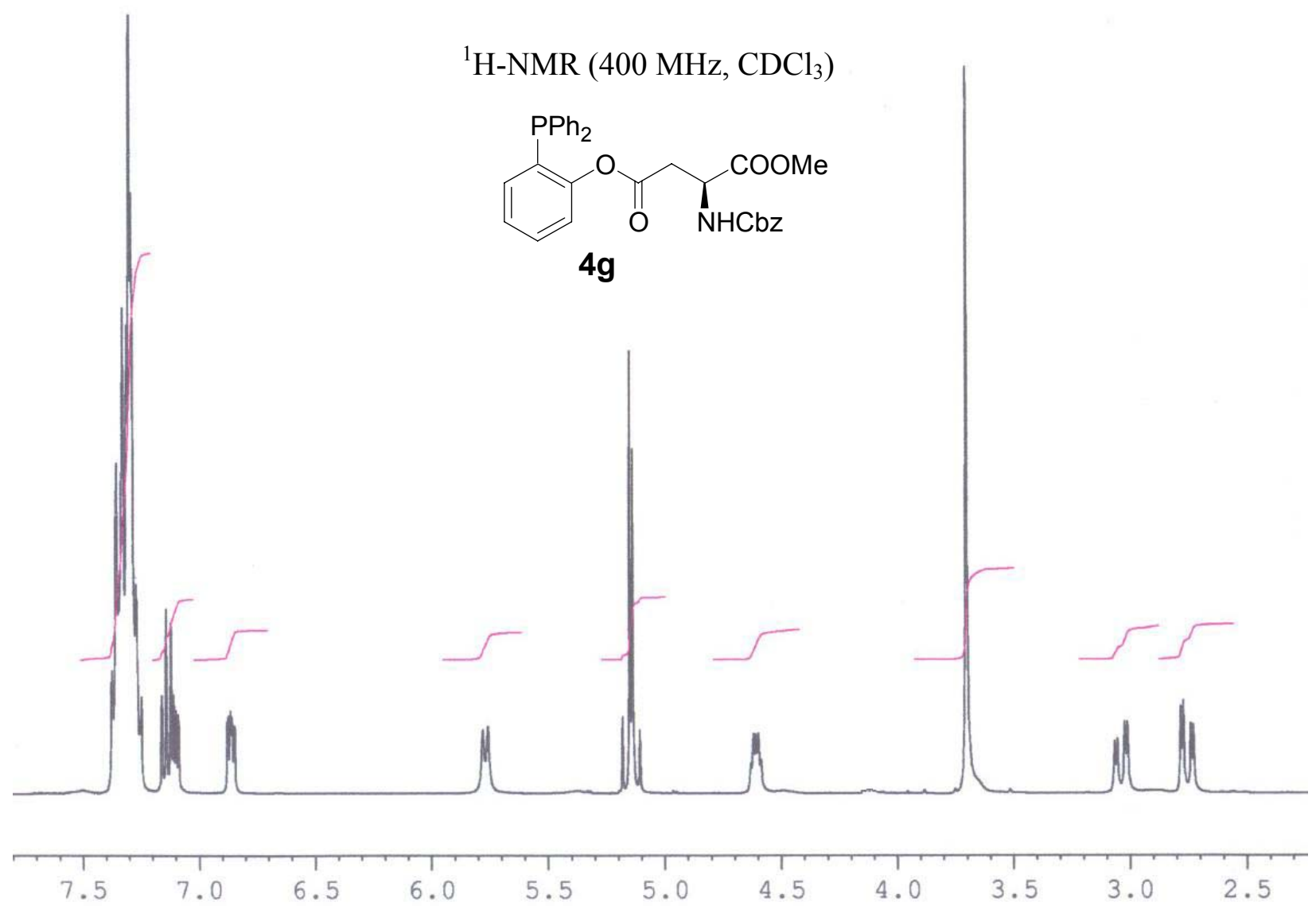

${ }^{31} \mathrm{P}-\mathrm{NMR}\left(161 \mathrm{MHz}, \mathrm{CDCl}_{3}\right)$<smiles>CC(=O)NC(CC(=O)Oc1ccccc1-c1ccccc1)C(C)=O</smiles>

49 
$N$-t-Butoxycarbonyl-L-aspartic acid 4-(2-diphenylphosphanyl-phenyl) ester 1-benzyl ester (4h)
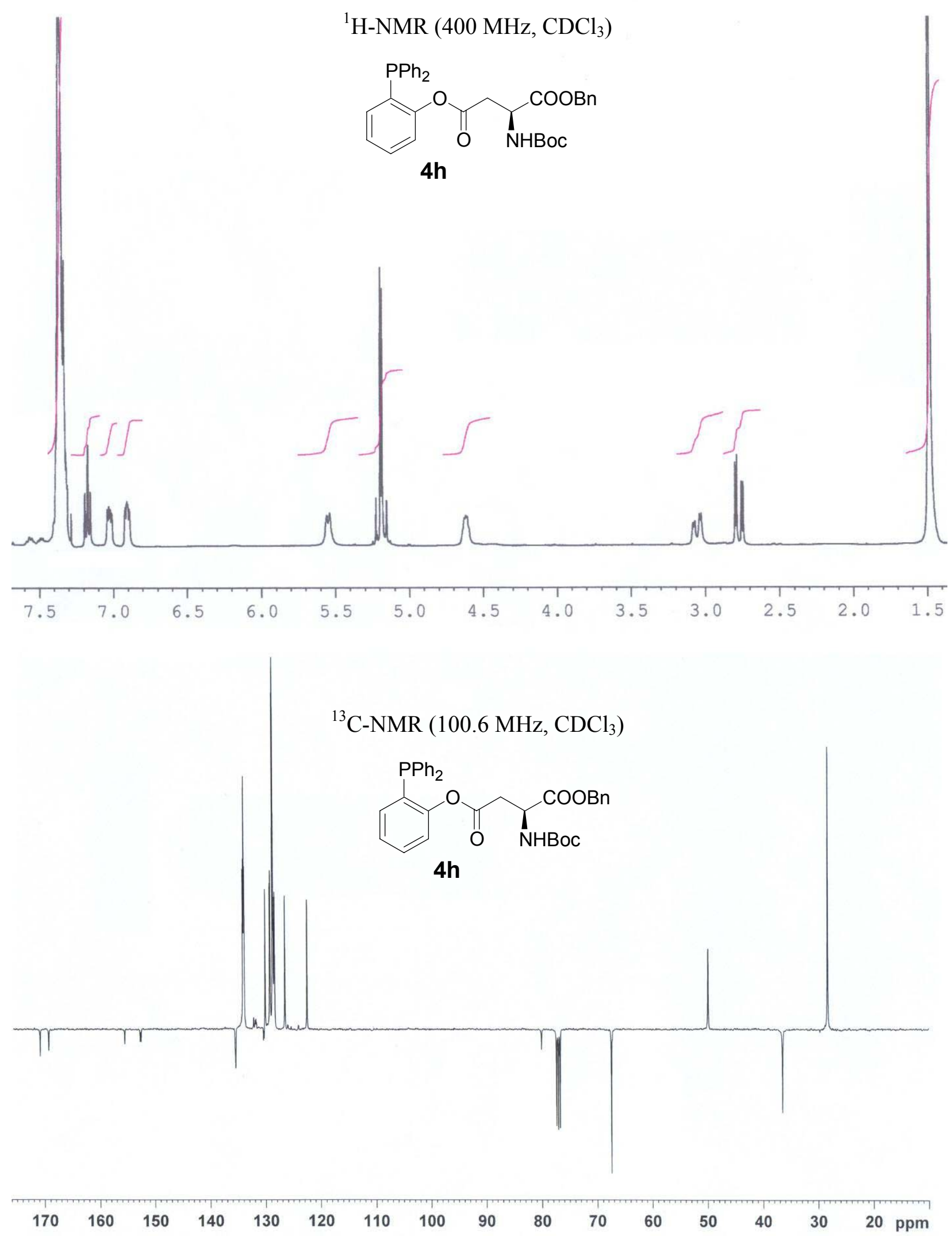

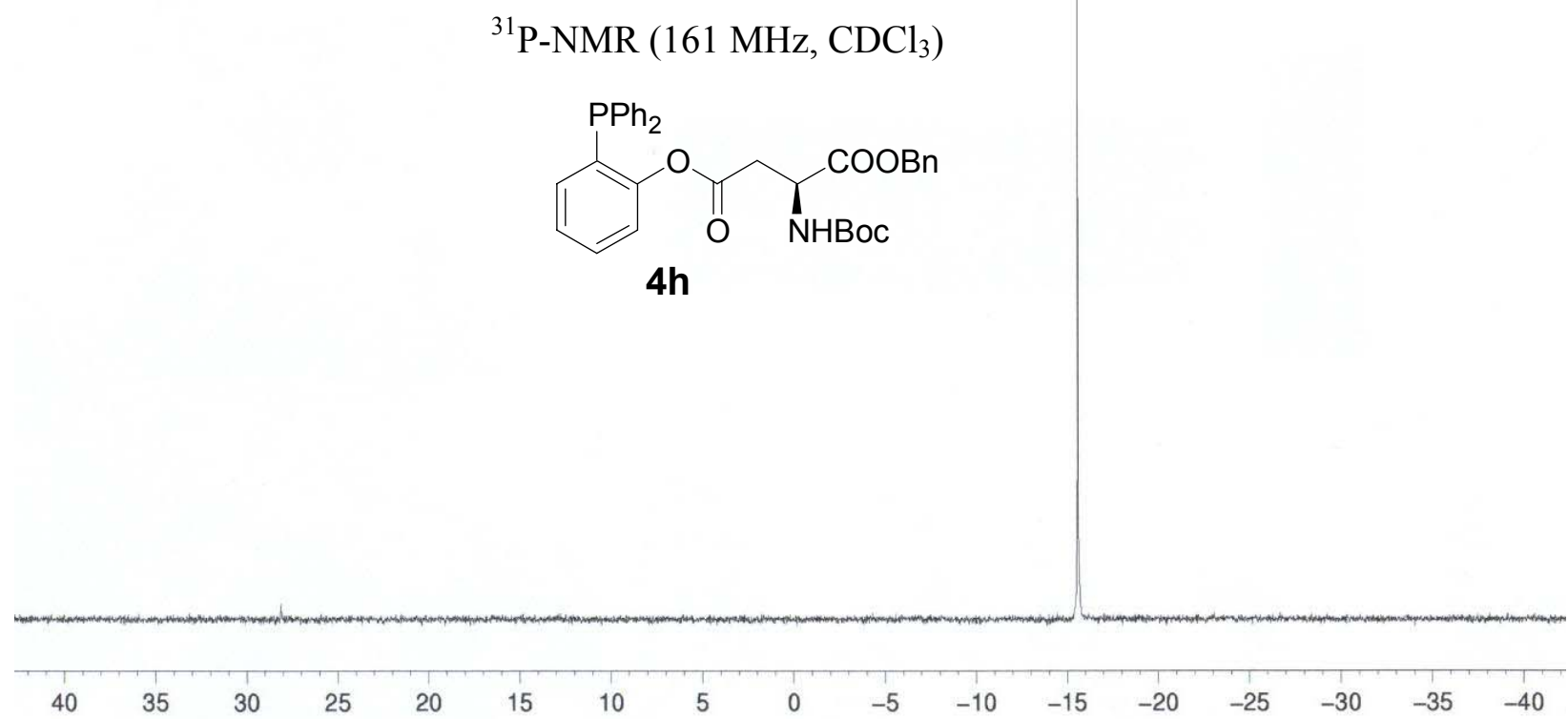

$\mathrm{N}$-Fluoren-9-ylmethoxycarbonyl-L-aspartic acid 4-(2-diphenylphosphanyl-phenyl) ester 1-benzyl ester (4i)

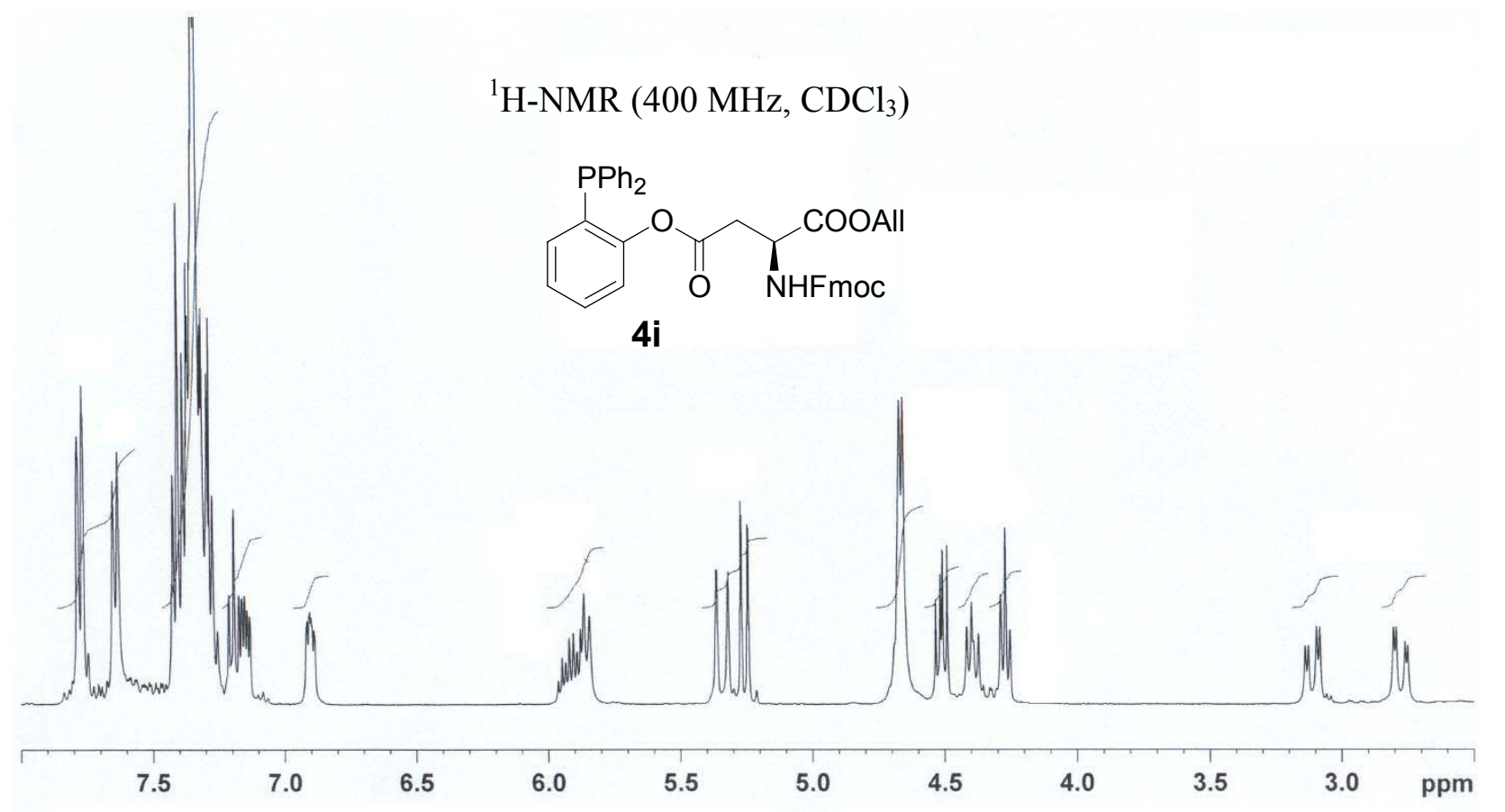



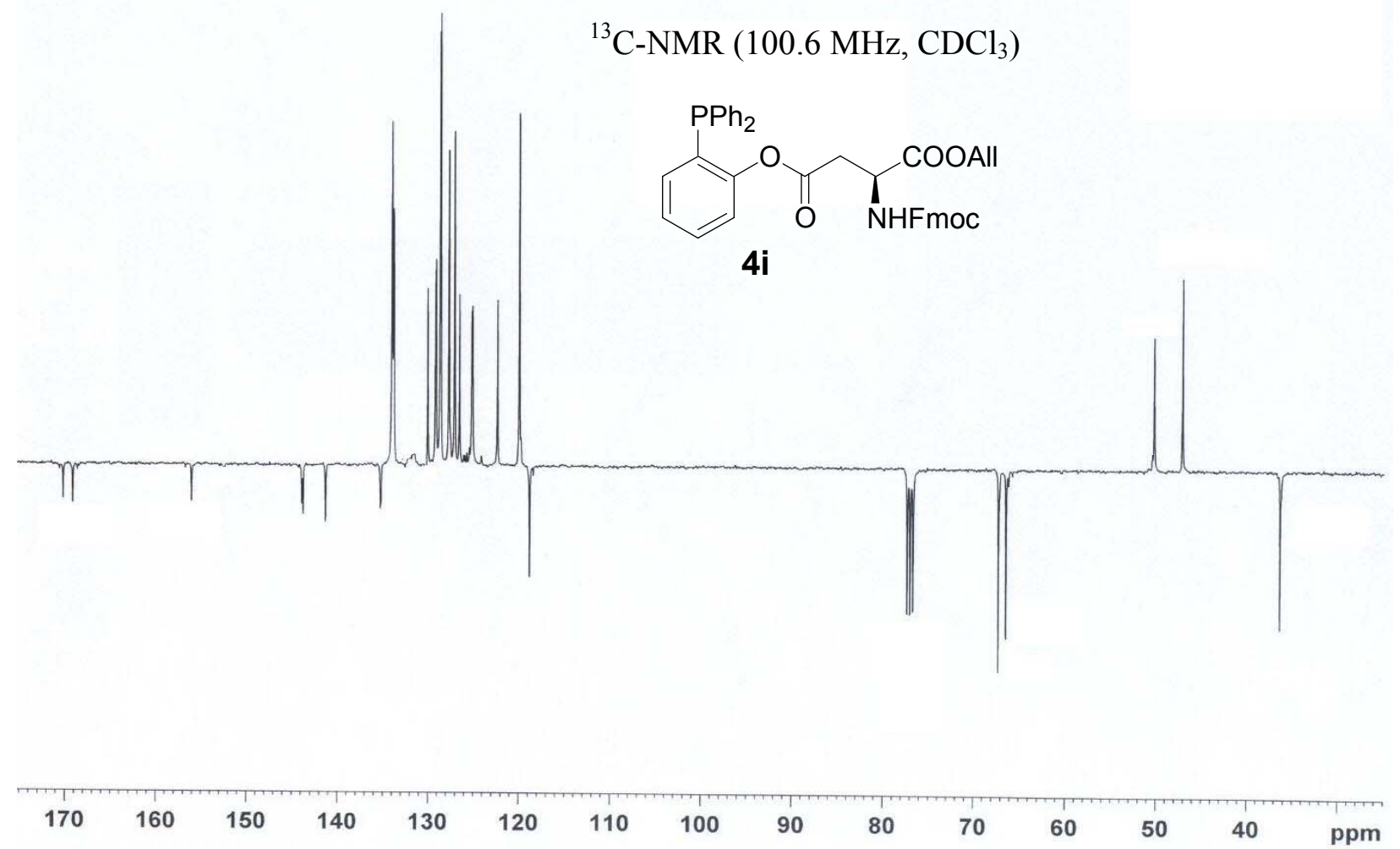

${ }^{31} \mathrm{P}-\mathrm{NMR}\left(161 \mathrm{MHz}, \mathrm{CDCl}_{3}\right.$ )<smiles>O=C(CC(N=CP)C(=O)OCc1ccccc1)Oc1ccccc1-c1ccccc1</smiles>

$4 i$ 
$N$ - Pentanedioic acid -2,3,4,6-tetra-O-benzyl- $\alpha$-D-glucopyranosylamide methyl ester (11f)

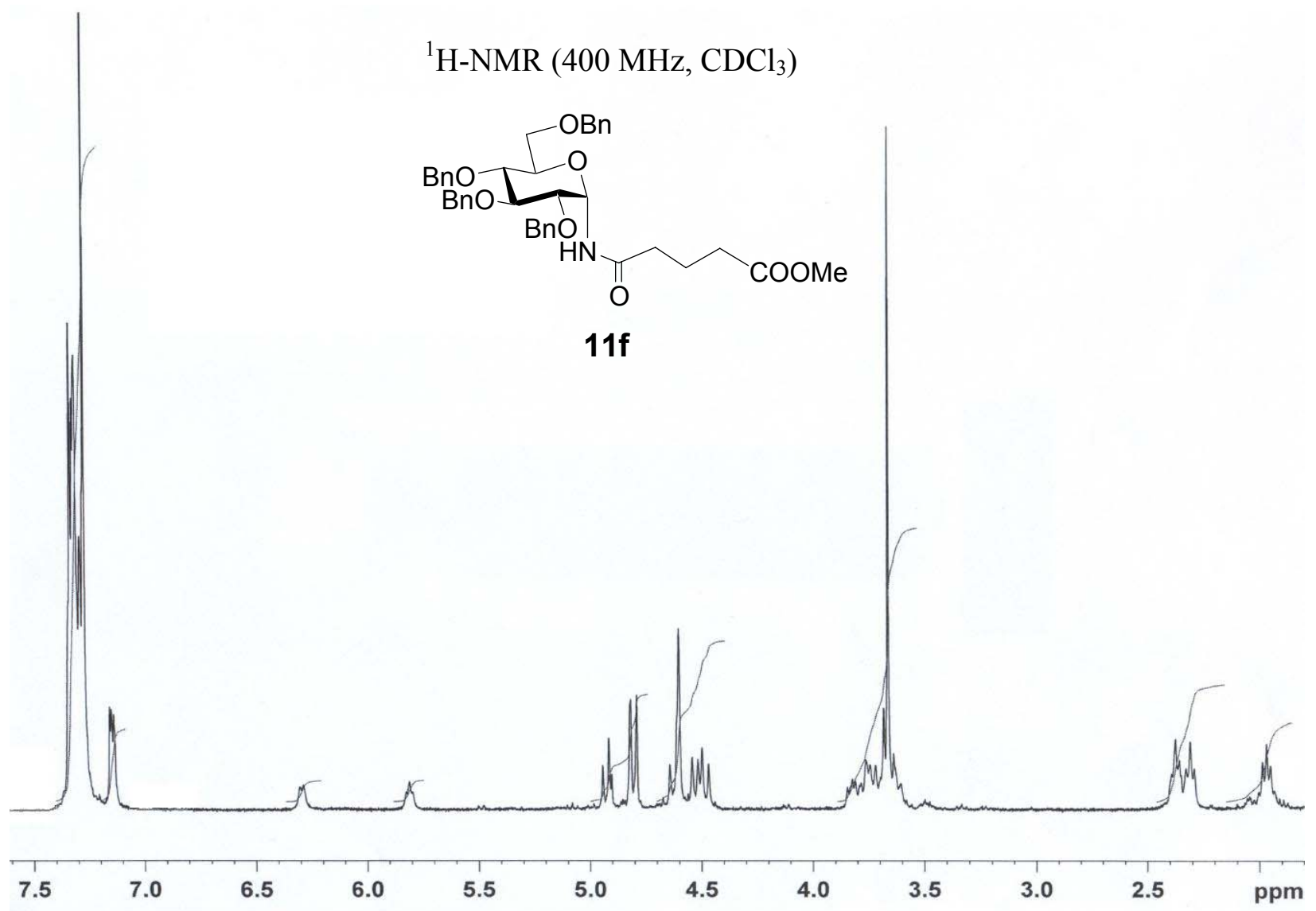

${ }^{13} \mathrm{C}-\mathrm{NMR}\left(100.6 \mathrm{MHz}, \mathrm{CDCl}_{3}\right.$ )

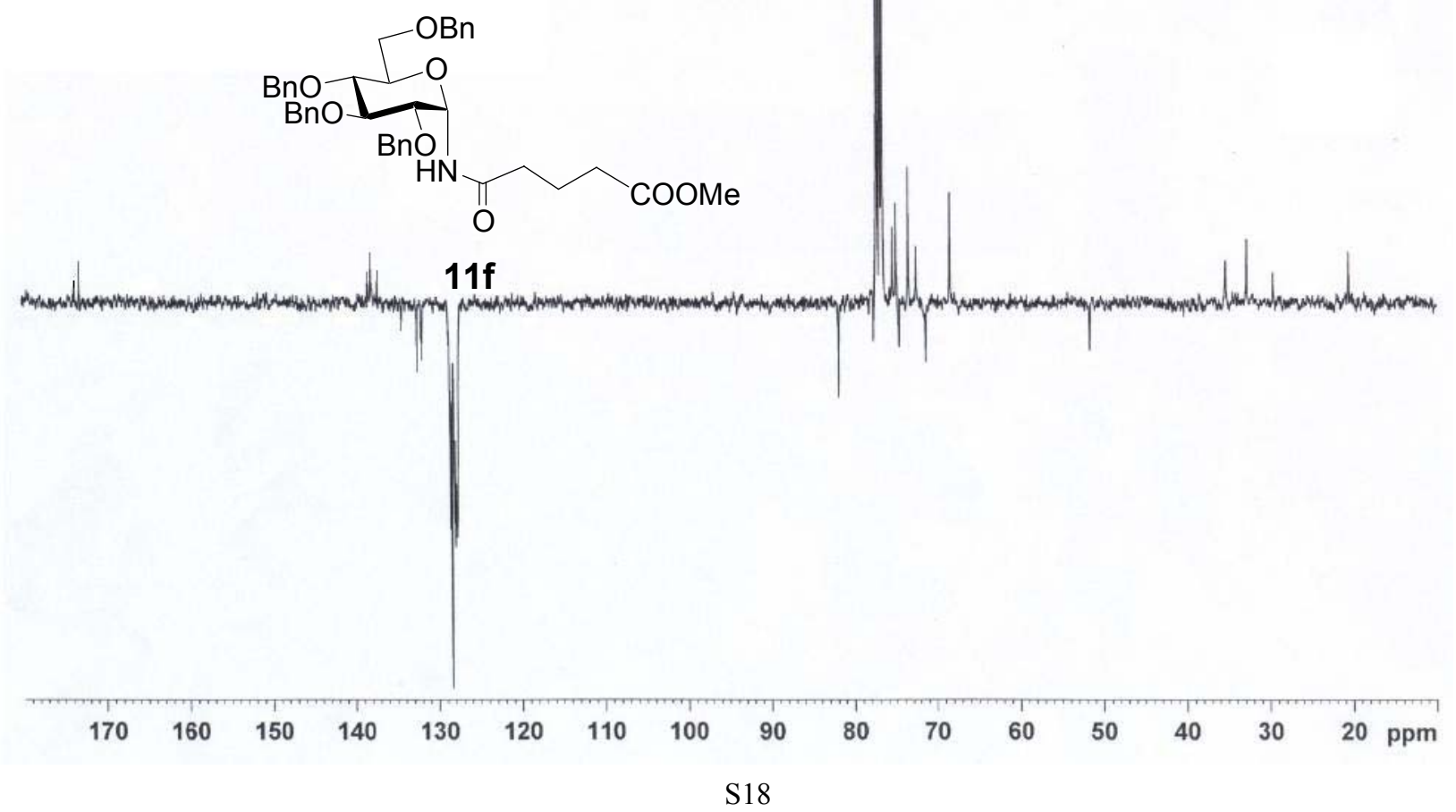


$N^{\alpha}$-benzyloxycarbonyl- $N^{\gamma}$-(2,3,4,6-tetra- $O$-benzyl- $\alpha$-D-glucopyranosyl)-L-asparagine- $O$-methyl ester (11g)
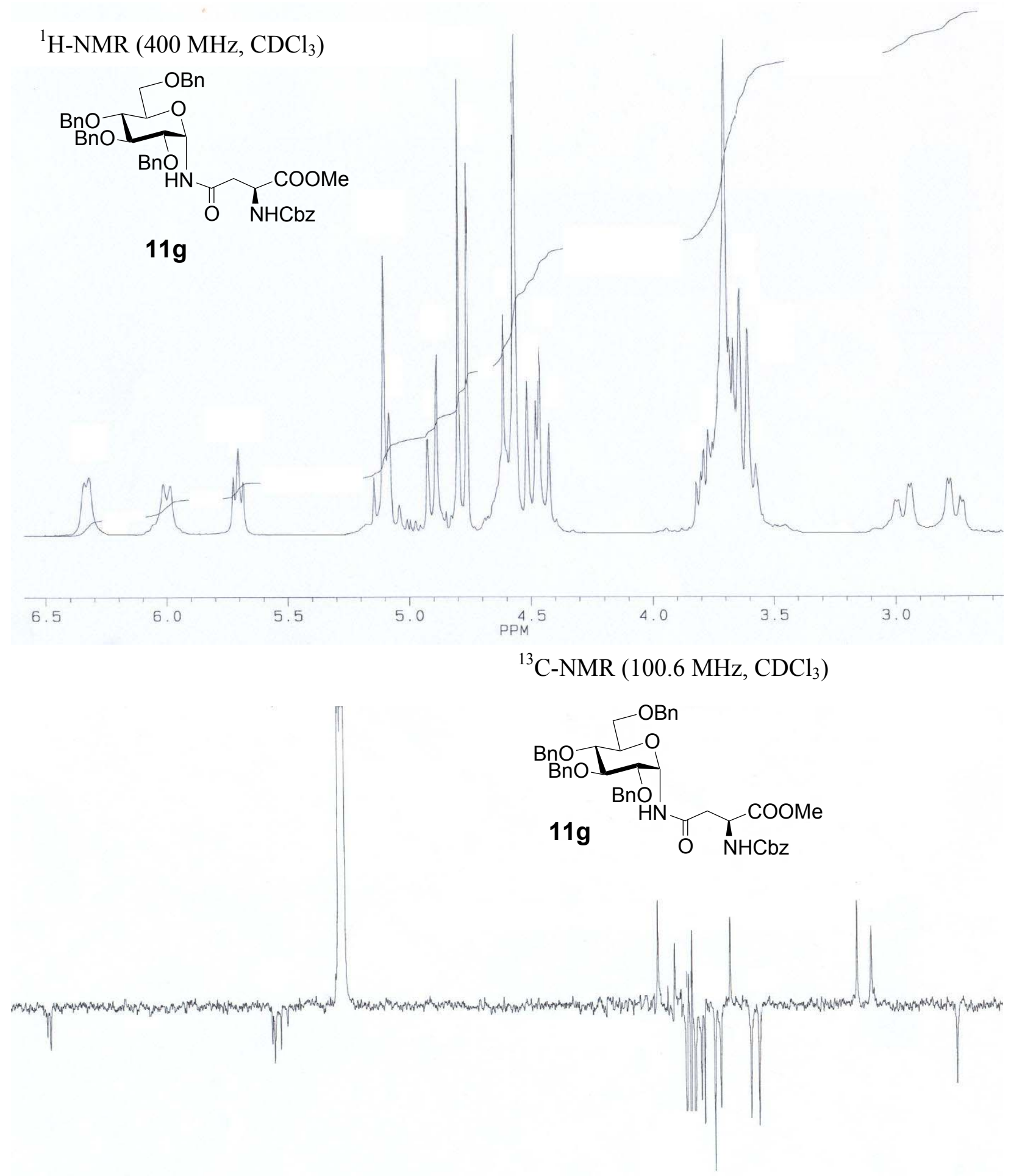
COSY $\left(400 \mathrm{MHz}, \mathrm{CDCl}_{3}\right)$

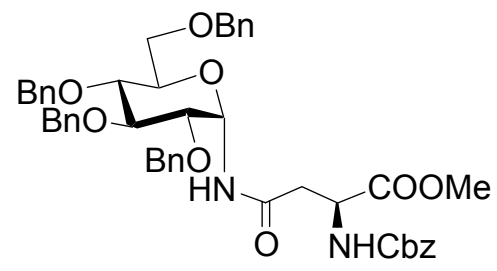

$11 \mathrm{~g}$

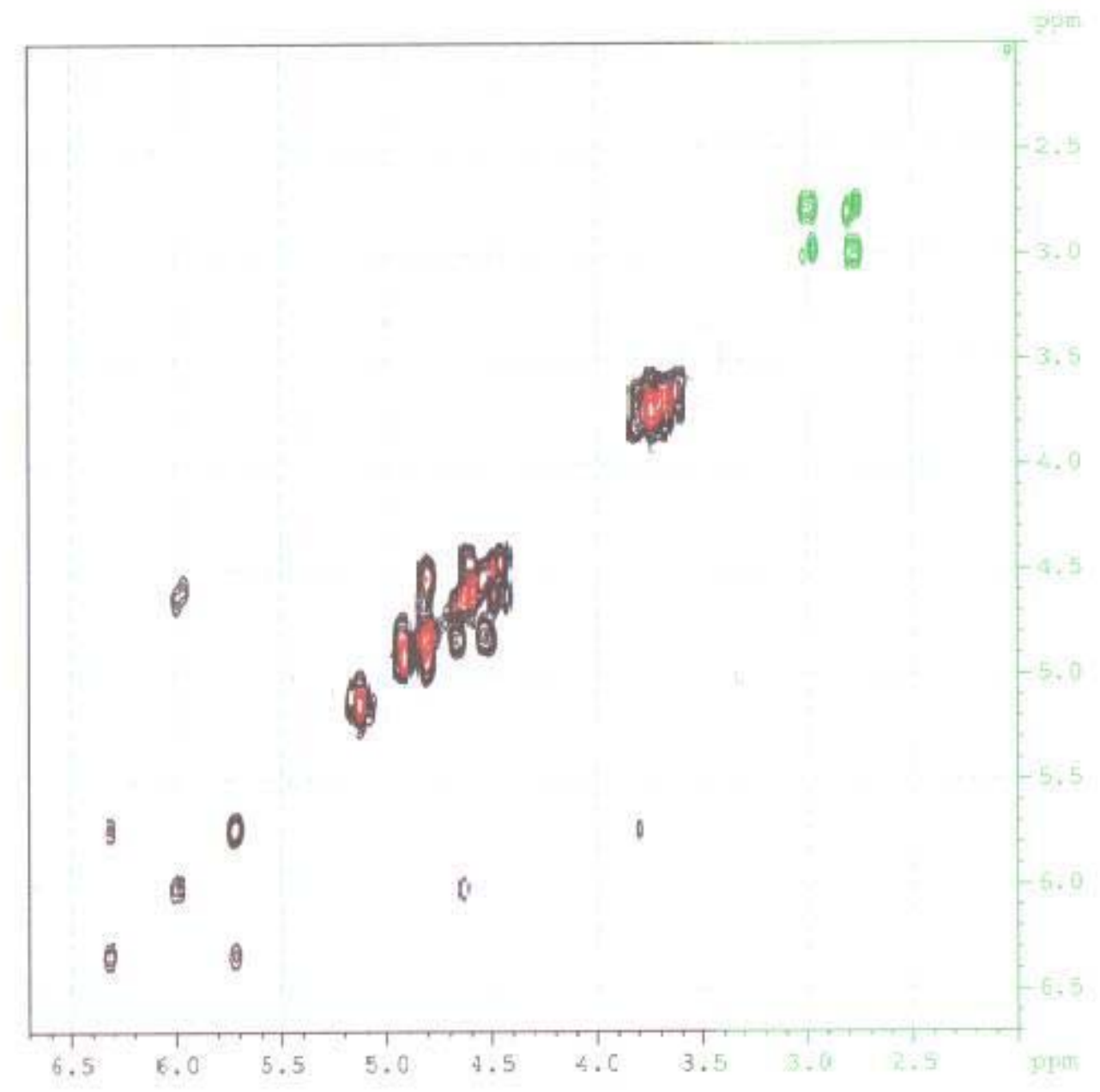


$N^{\alpha}$-t-butoxycarbonyl-N ${ }^{\gamma}$-(2,3,4,6-tetra- $O$-benzyl- $\alpha$-D-glucopyranosyl)-L-asparagine- $O$-benzyl ester (11h)

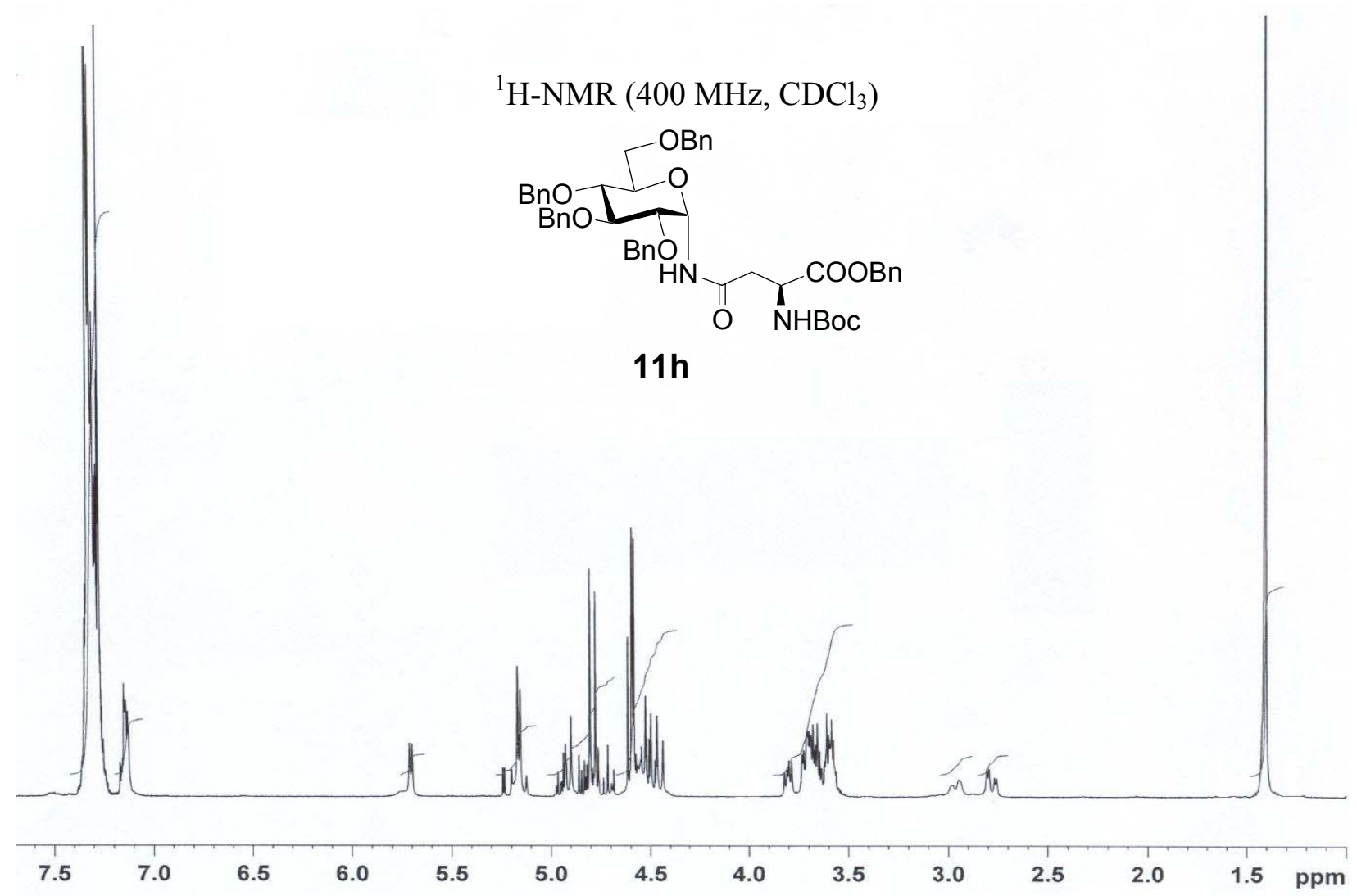

${ }^{13} \mathrm{C}-\mathrm{NMR}\left(100.6 \mathrm{MHz}, \mathrm{CDCl}_{3}\right)$

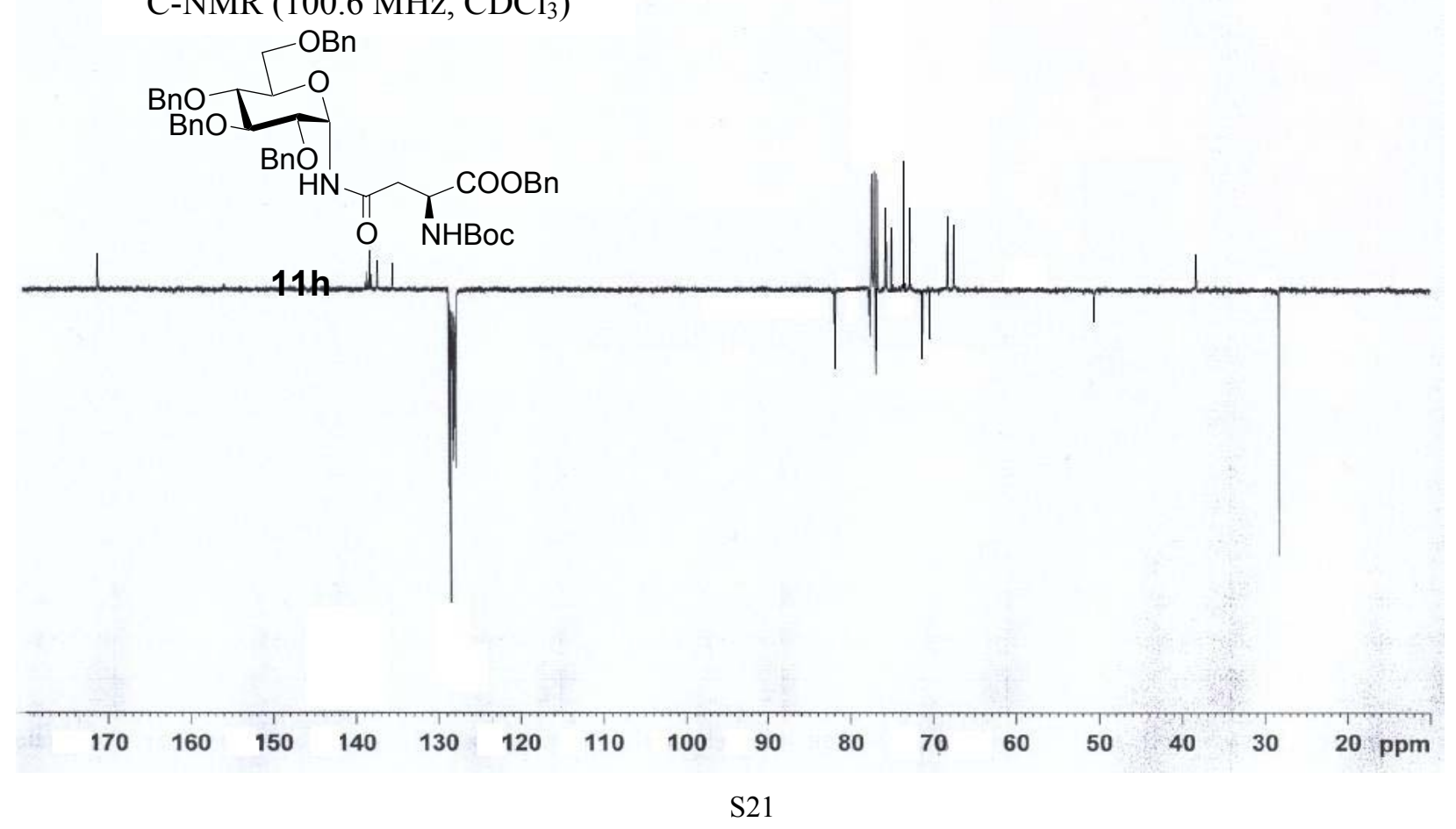




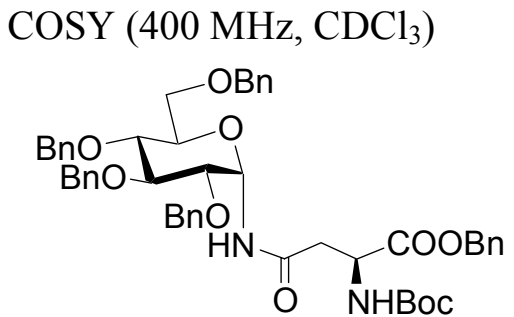

\section{1h}

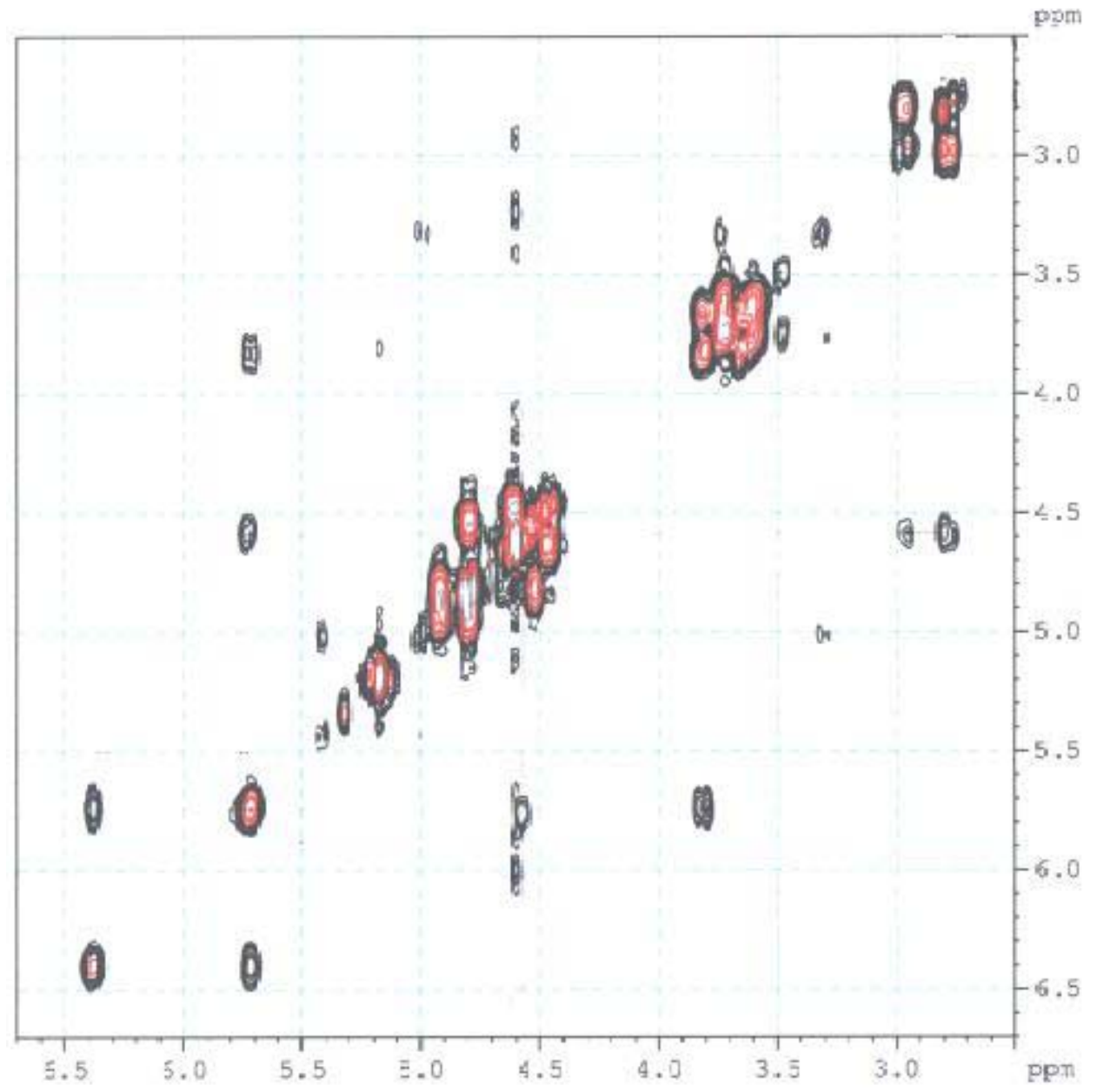


$N^{\alpha}$-fluoren-9-ylmethoxycarbonyl- $N^{\gamma}$-(2,3,4,6-tetra- $O$-benzyl- $\alpha$-D-glucopyranosyl)-L-asparagine$O$-allyl ester (11i)

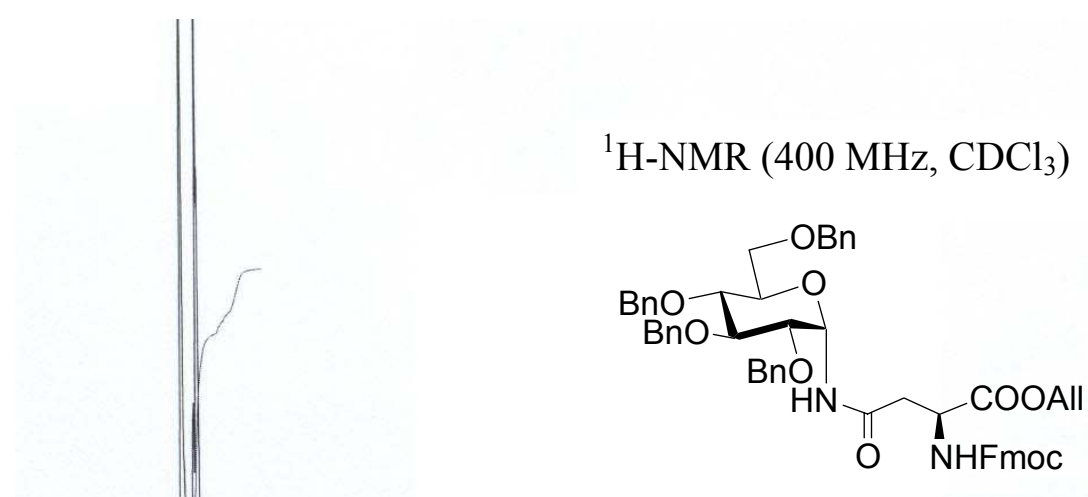

$11 \mathbf{i}$

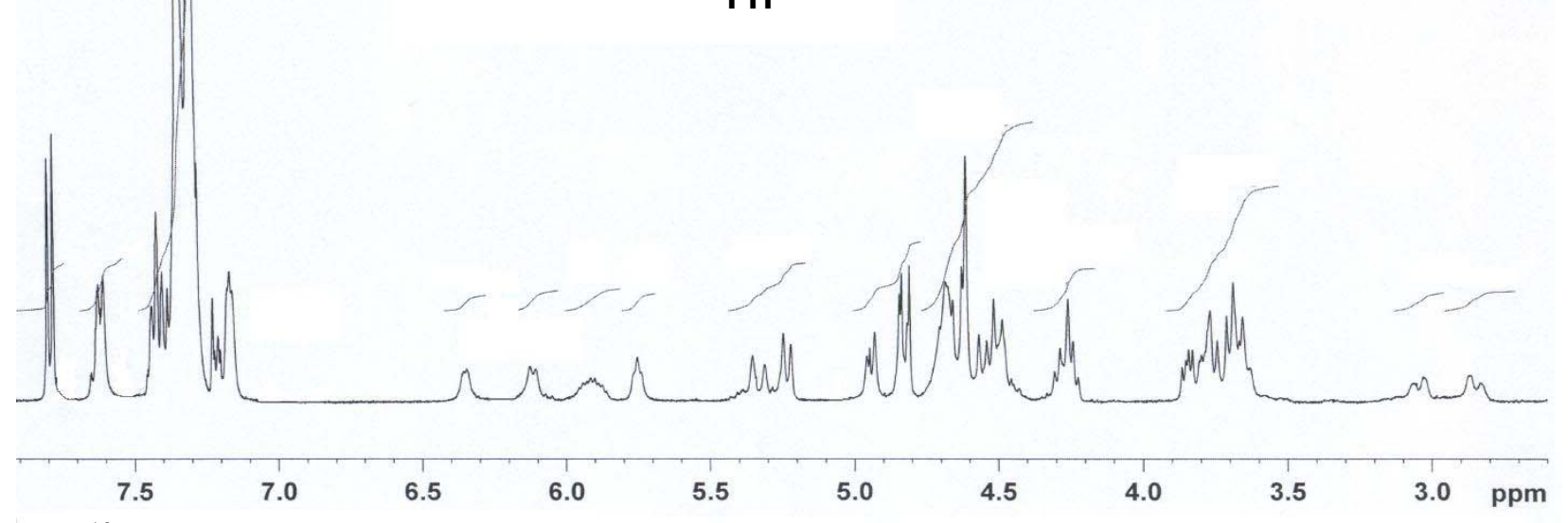

${ }^{13} \mathrm{C}-\mathrm{NMR}\left(100.6 \mathrm{MHz}, \mathrm{CDCl}_{3}\right)$

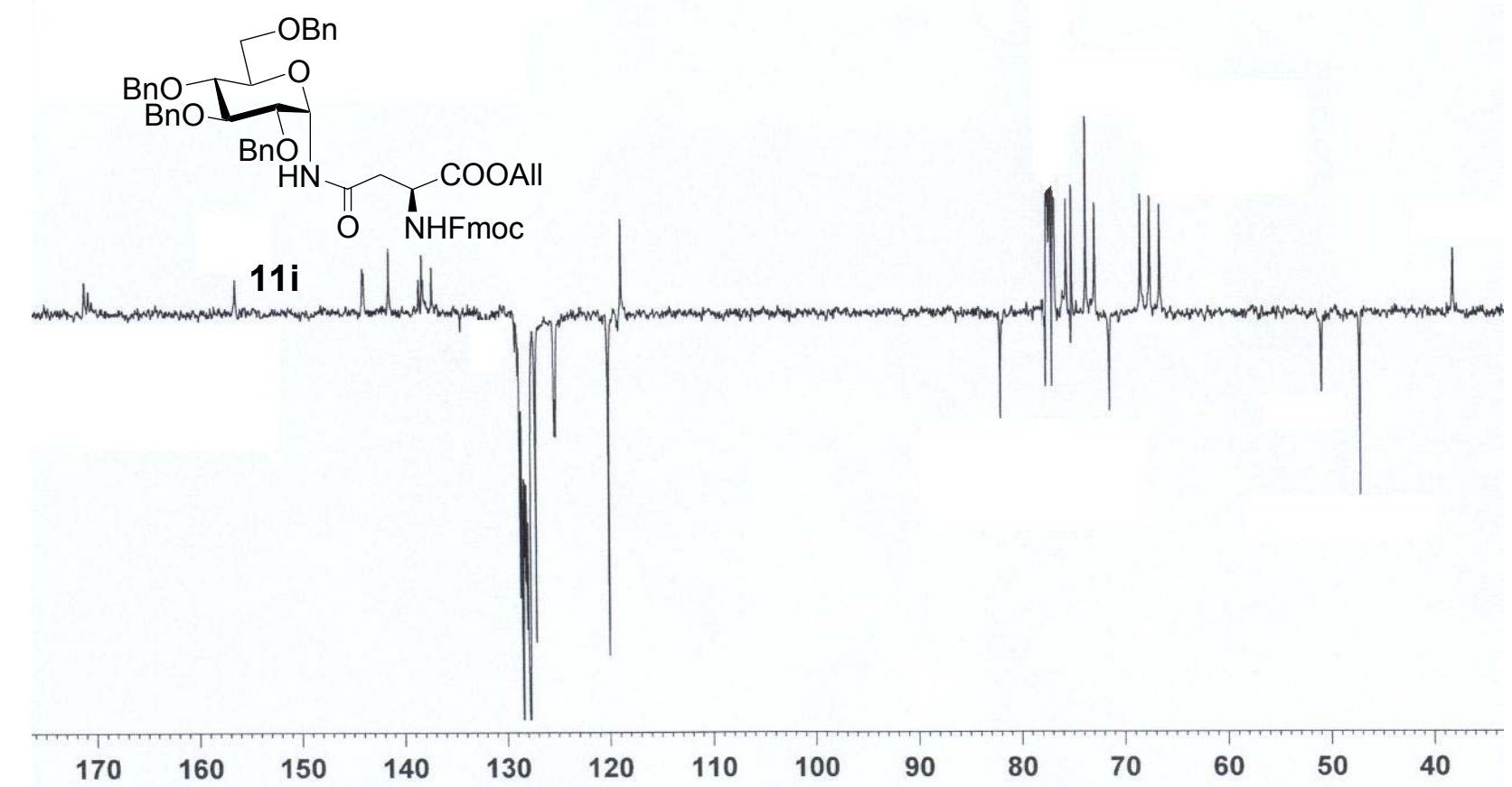


$\operatorname{COSY}\left(400 \mathrm{MHz}, \mathrm{CDCl}_{3}\right)$

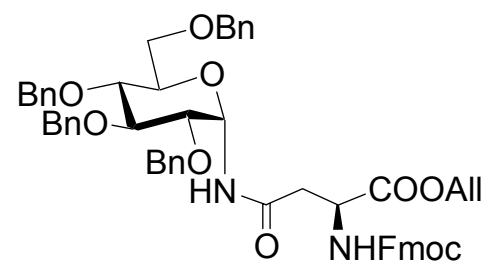

$11 \mathbf{i}$

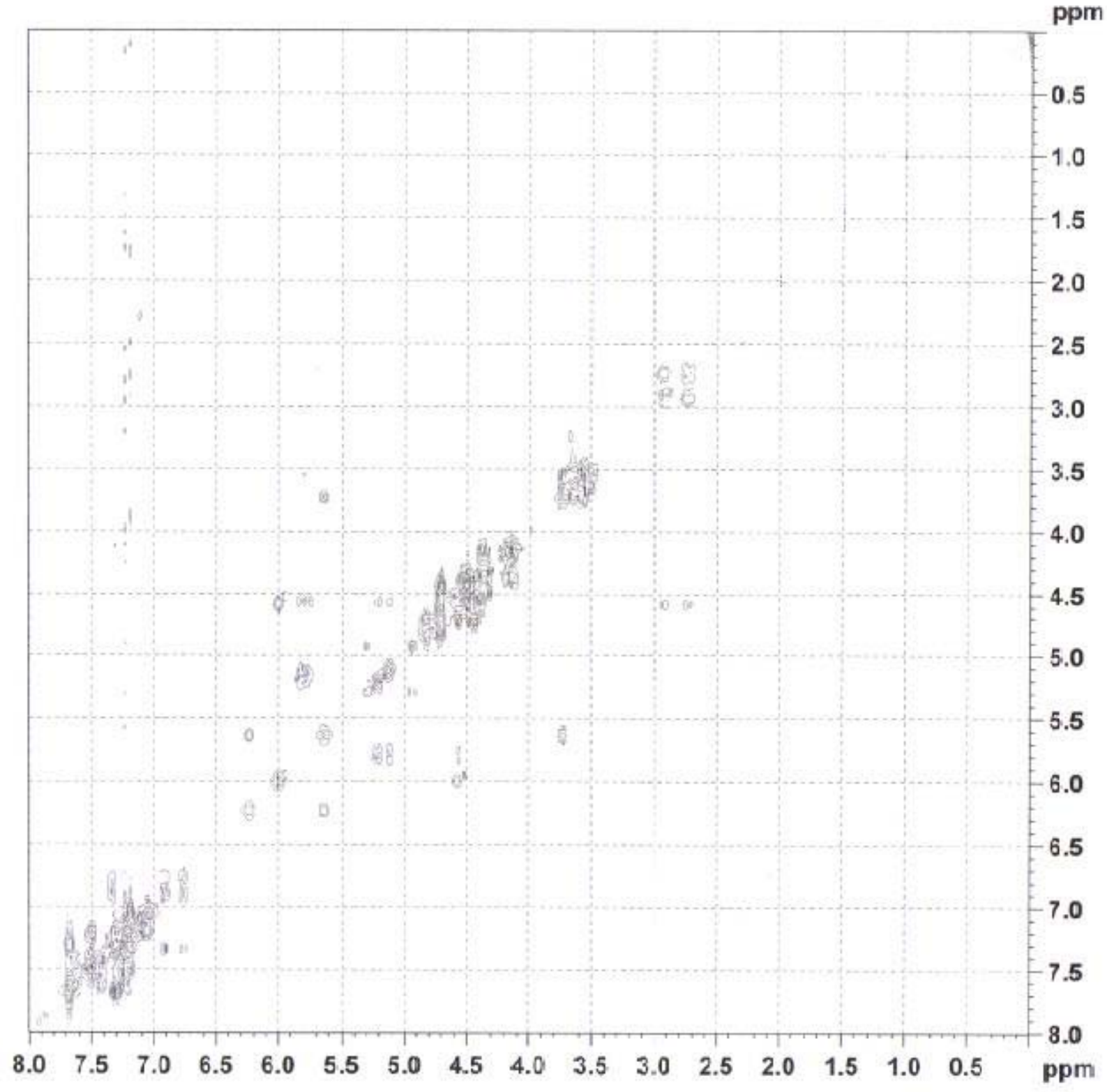


$N$-(3-Methylbutanoyl)-2,3,4,6-tetra- $O$-benzyl- $\alpha$-D-galactopyranosylamine (12c)

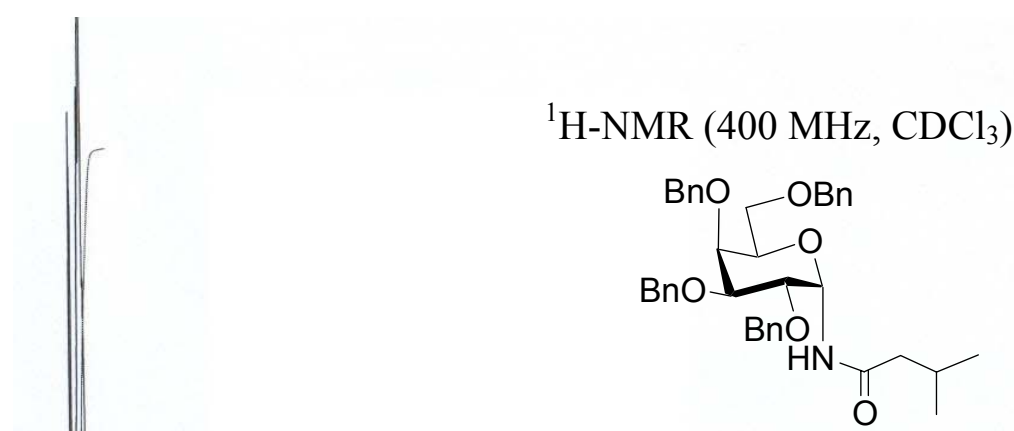

$12 c$

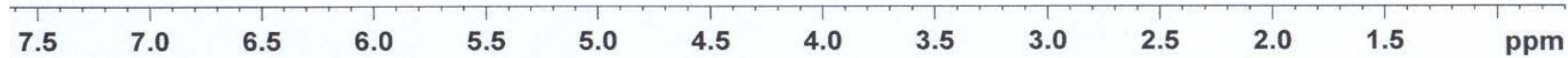

${ }^{13} \mathrm{C}-\mathrm{NMR}\left(100.6 \mathrm{MHz}, \mathrm{CDCl}_{3}\right)$

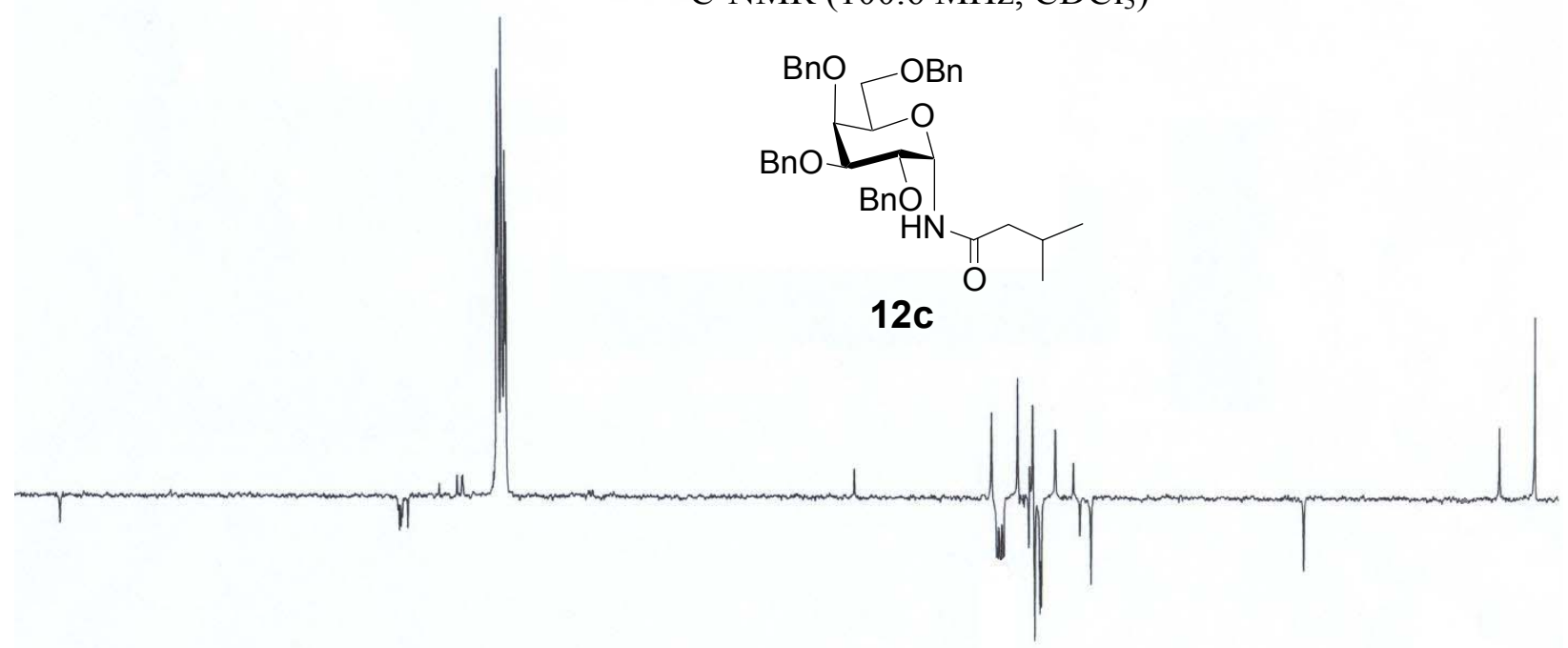

$\begin{array}{llllllllllllllllll}170 & 160 & 150 & 140 & 130 & 120 & 110 & 100 & 90 & 80 & 70 & 60 & 50 & 40 & 30 & \text { ppm }\end{array}$ 
COSY $\left(400 \mathrm{MHz}, \mathrm{CDCl}_{3}\right)$

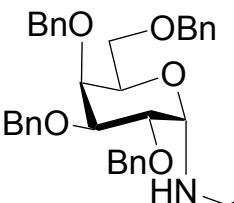

12c

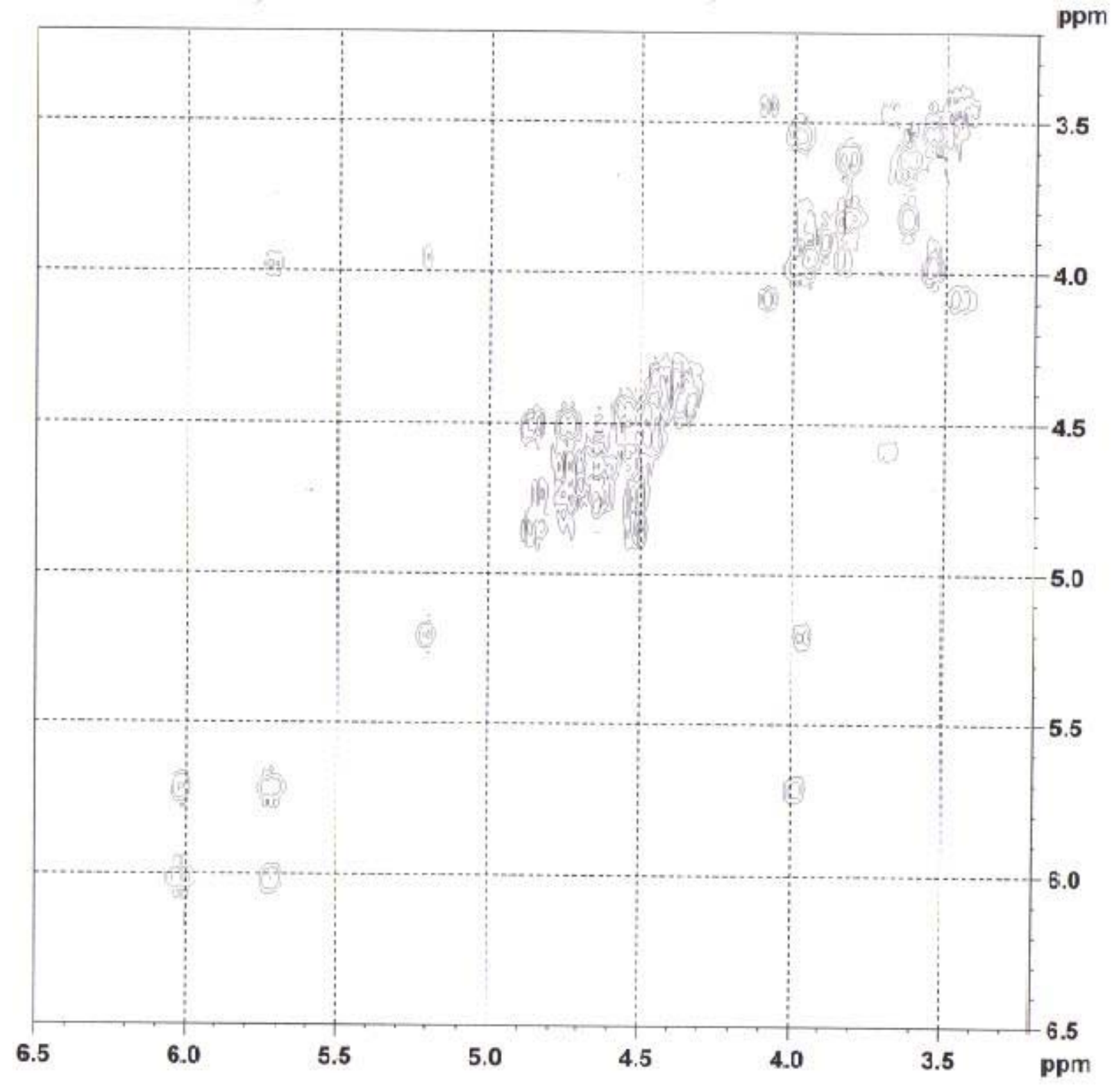


$N^{\alpha}$-benzyloxycarbonyl- $N^{\gamma}$-(2,3,4,6-tetra-O-benzyl- $\alpha / \beta$-D-galactopyranosyl)-L-asparagine- $O$ methyl ester (12g)

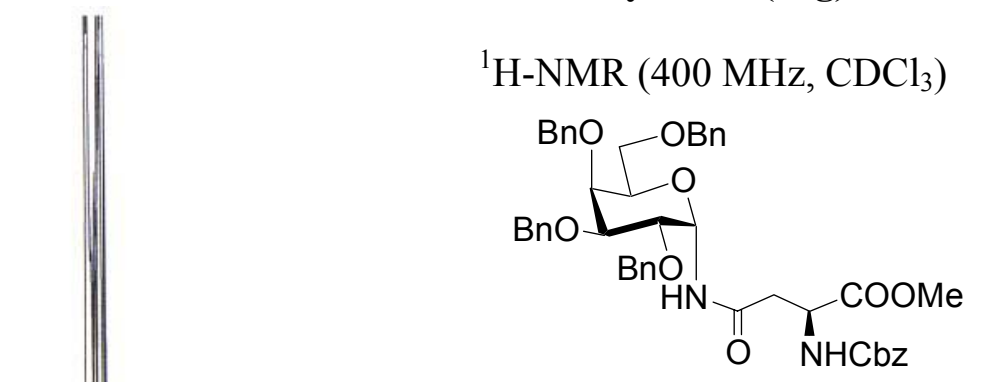

$12 \mathrm{~g}$

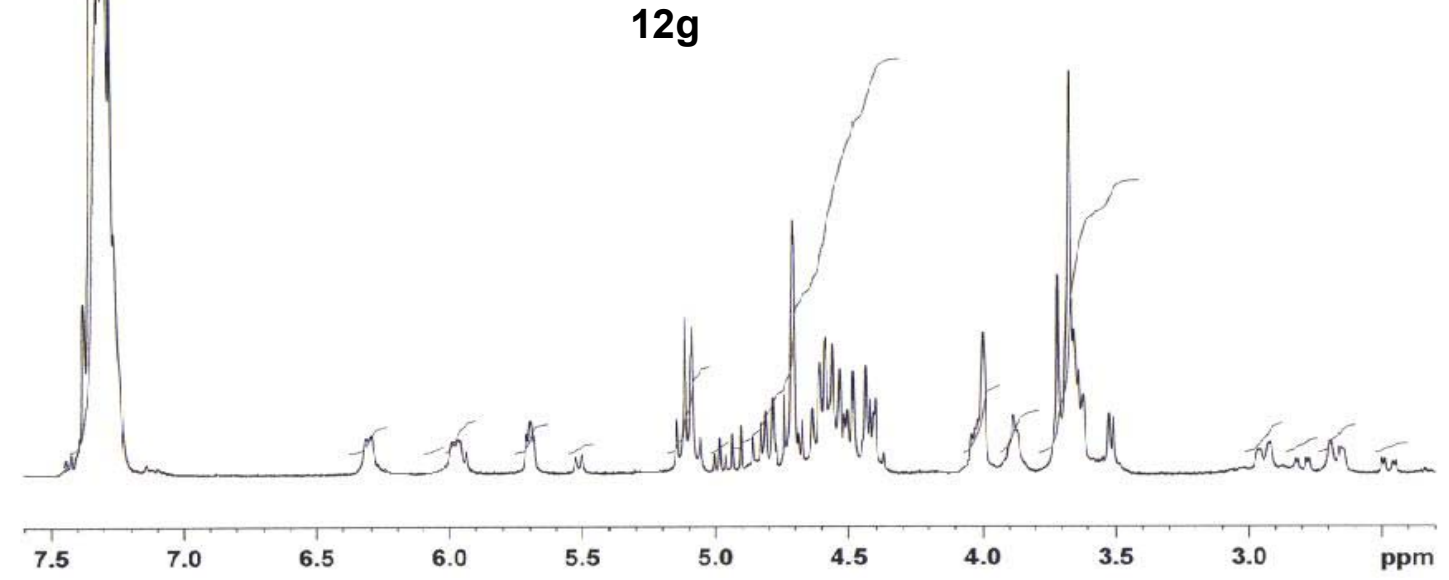

$N^{\alpha}$-t-butoxycarbonyl- $N^{\gamma}$-(2,3,4,6-tetra- $O$-benzyl- $\alpha / \beta$-D-galactopyranosyl)-L-asparagine- $O$-benzyl ester (12h)

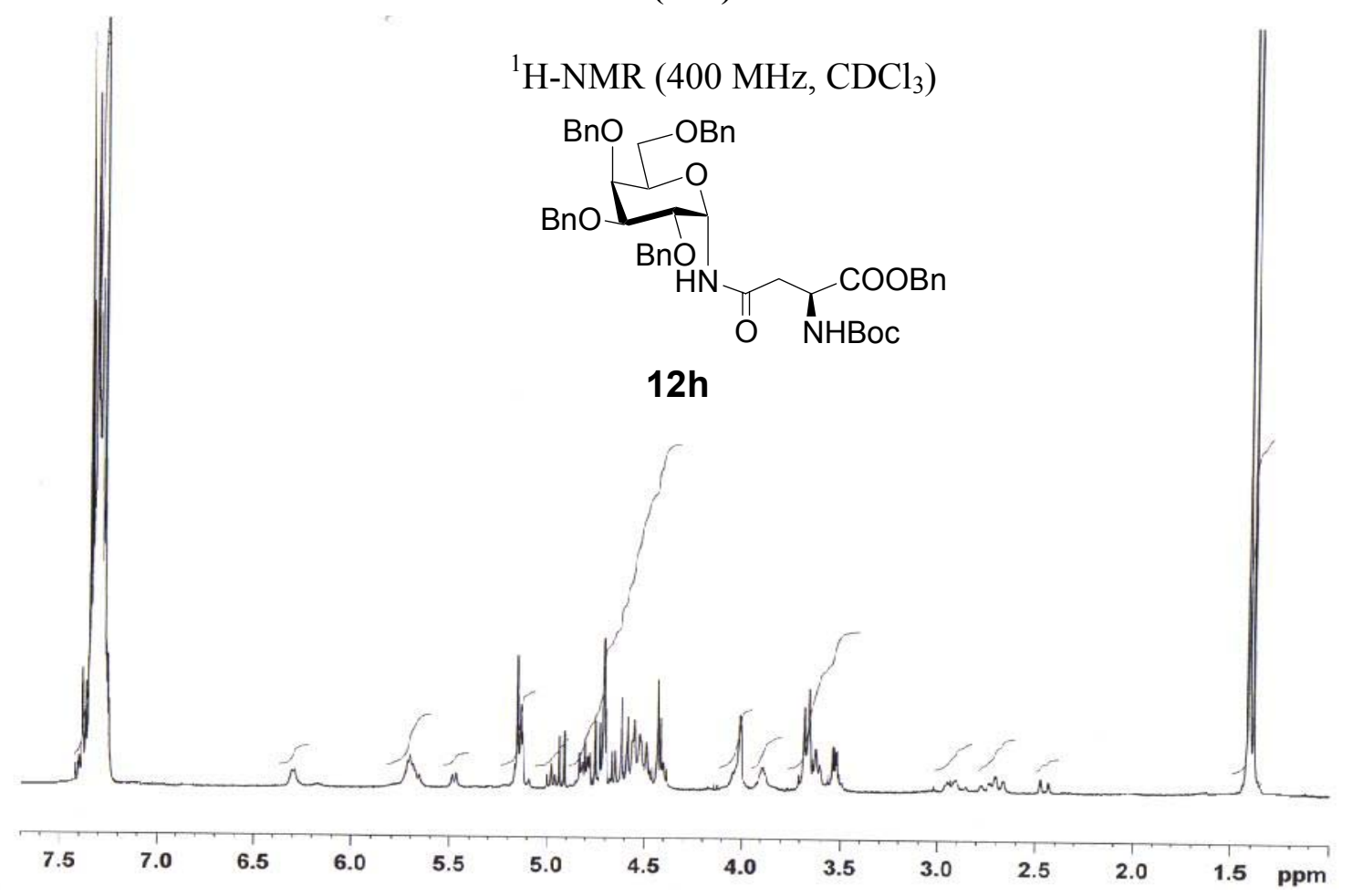




\section{$N$-Acetyl- $\alpha$-L-fucopyranosylamine (18a)}

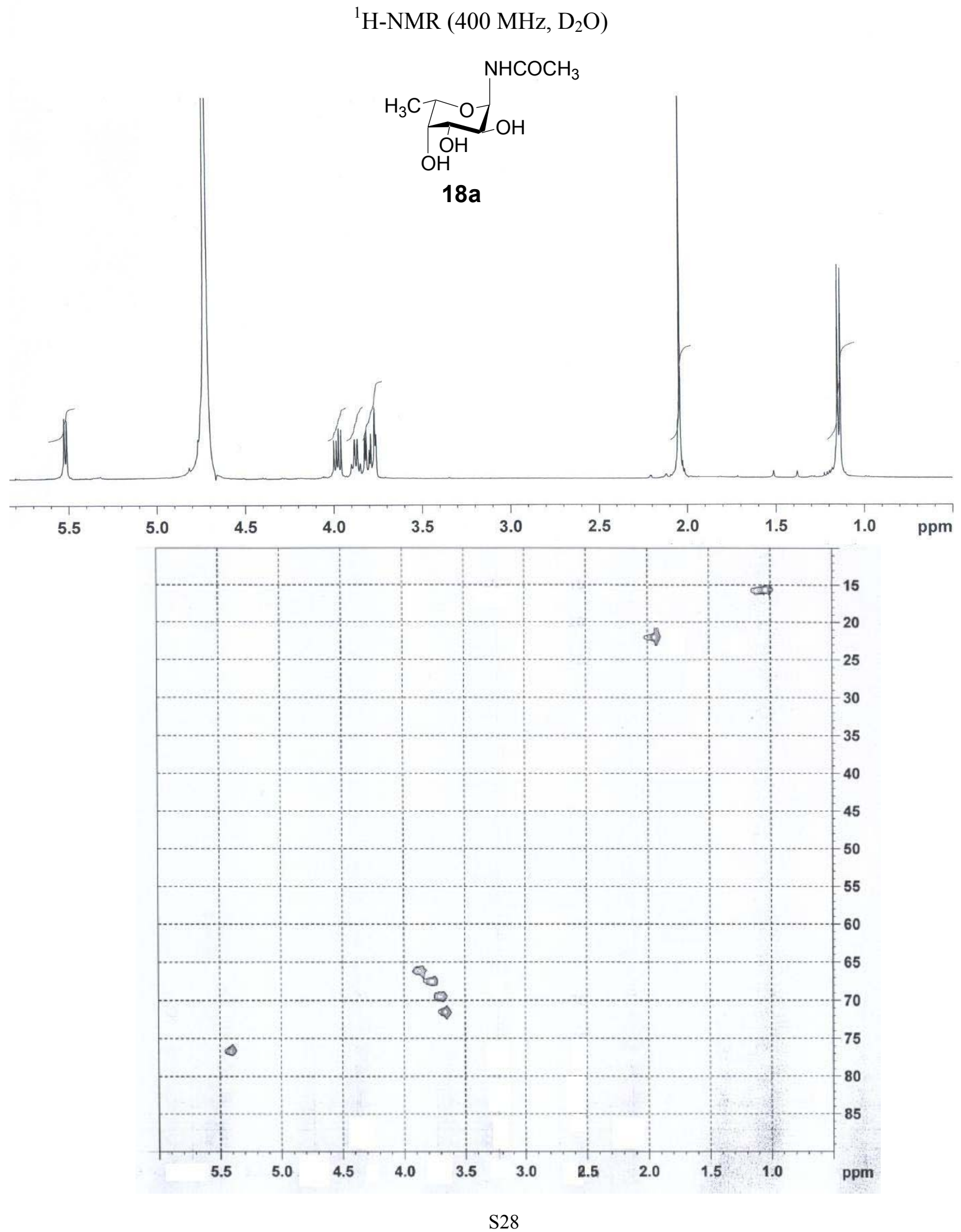




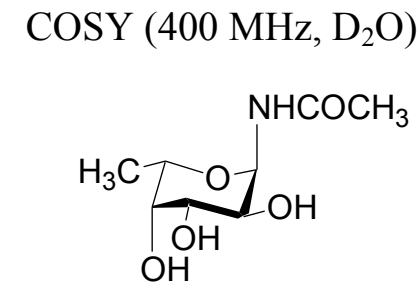

$18 a$

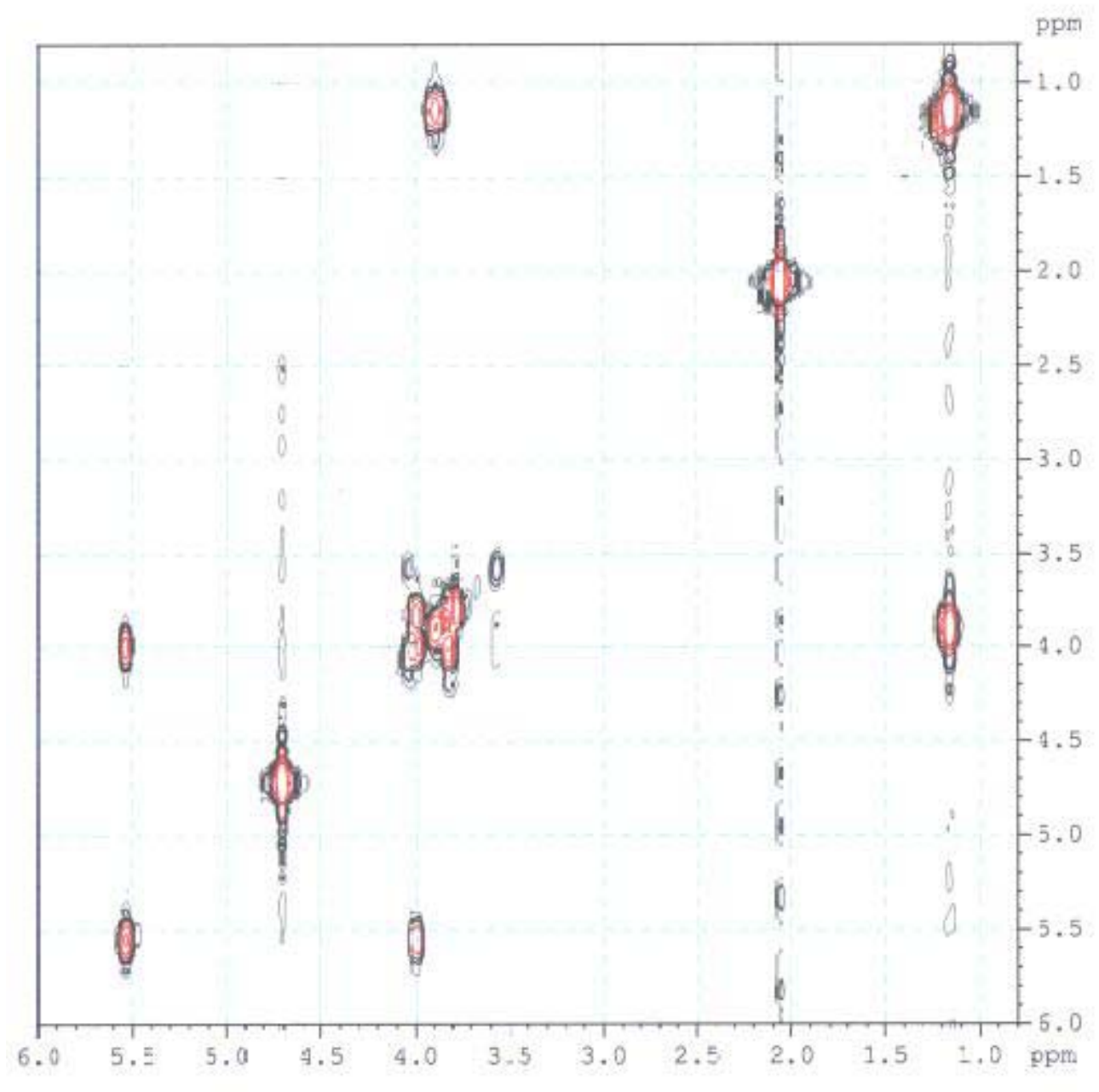


$N$-Acetyl- $\alpha$-D-glucopyranosylamine (19a)
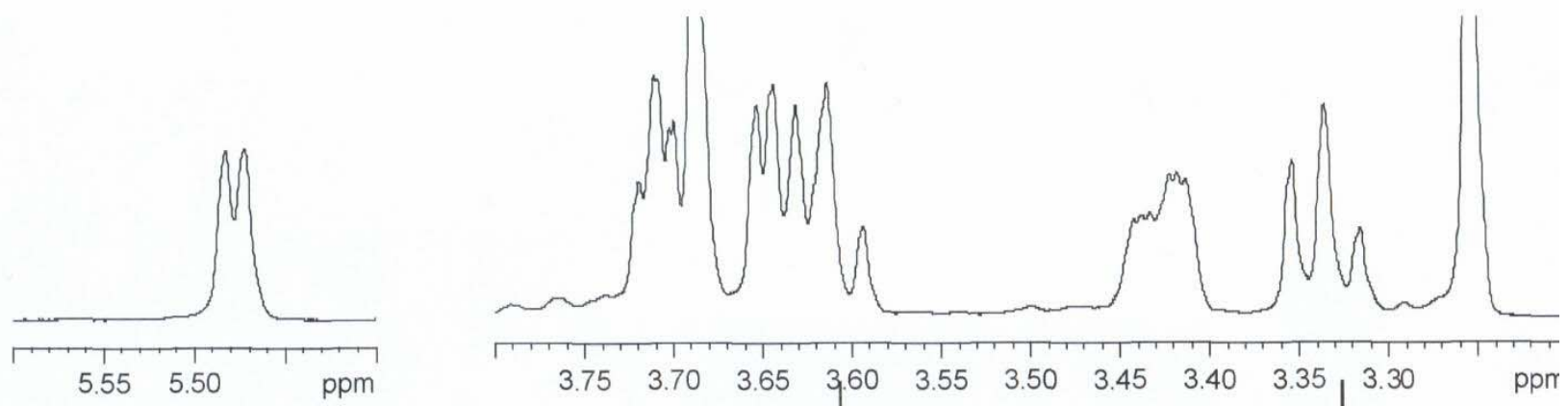

${ }^{1} \mathrm{H}-\mathrm{NMR}\left(500 \mathrm{MHz}, \mathrm{D}_{2} \mathrm{O}\right)$

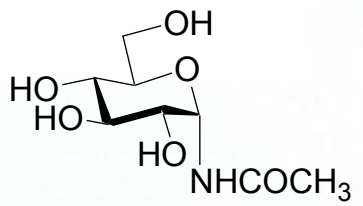

$19 a$
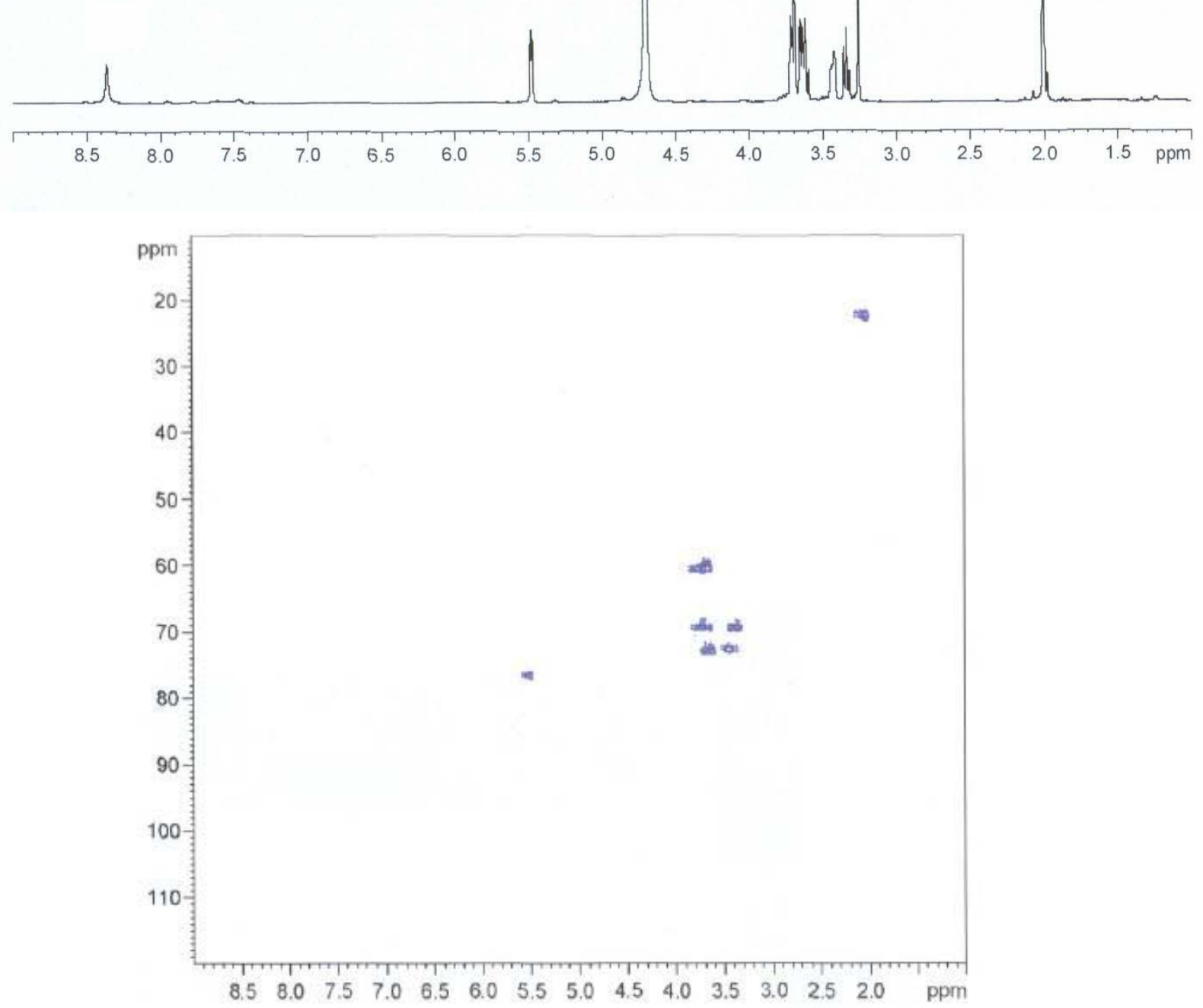
COSY (500 MHz, $\mathrm{D}_{2} \mathrm{O}$ )

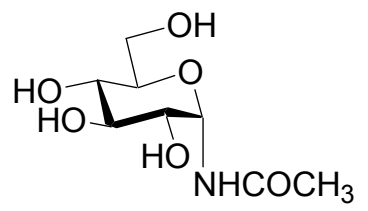

$19 a$

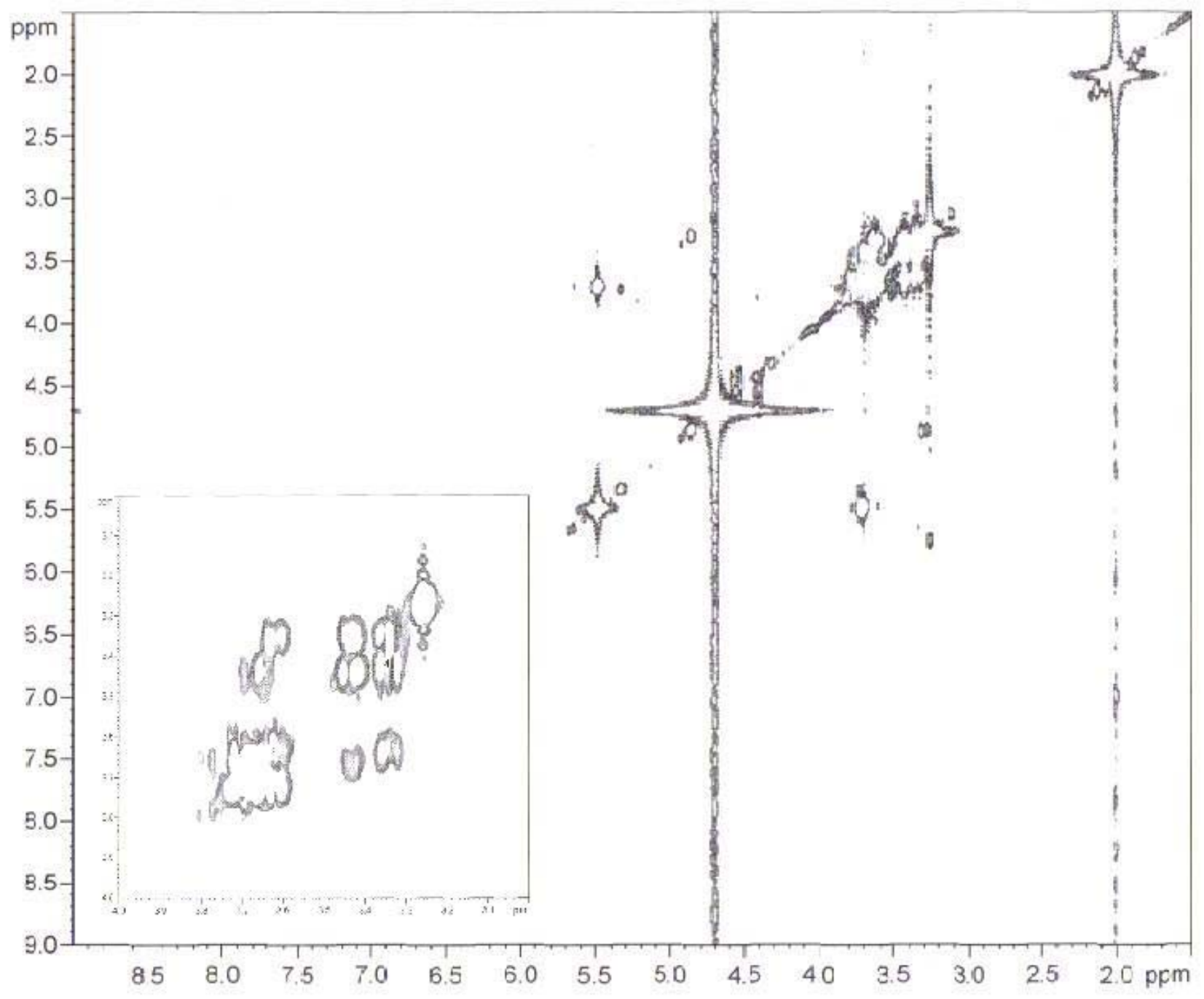




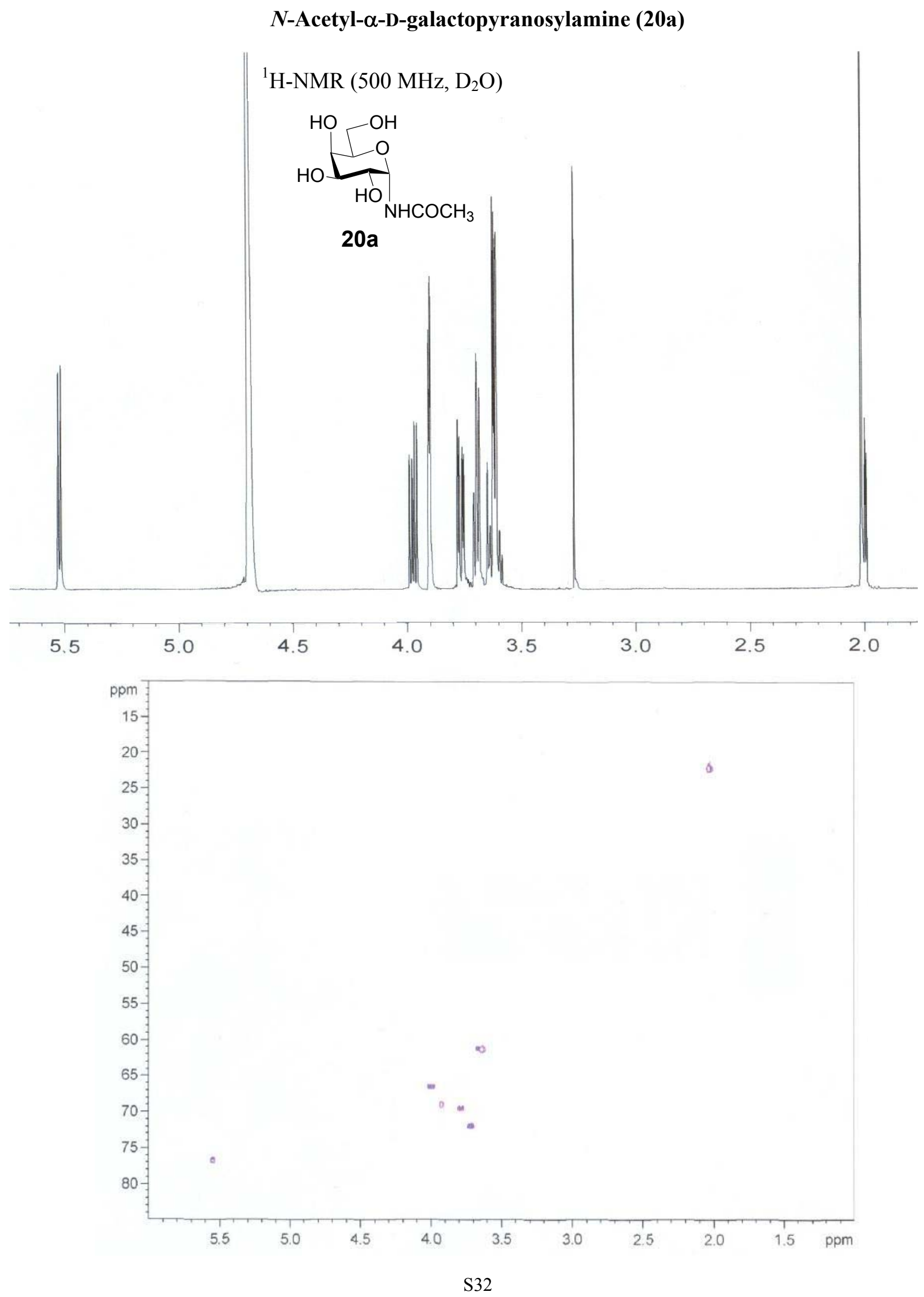


COSY (500 MHz, $\mathrm{D}_{2} \mathrm{O}$ )

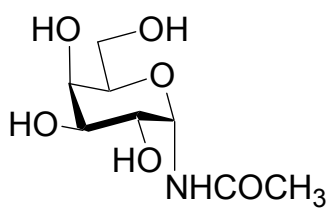

20a

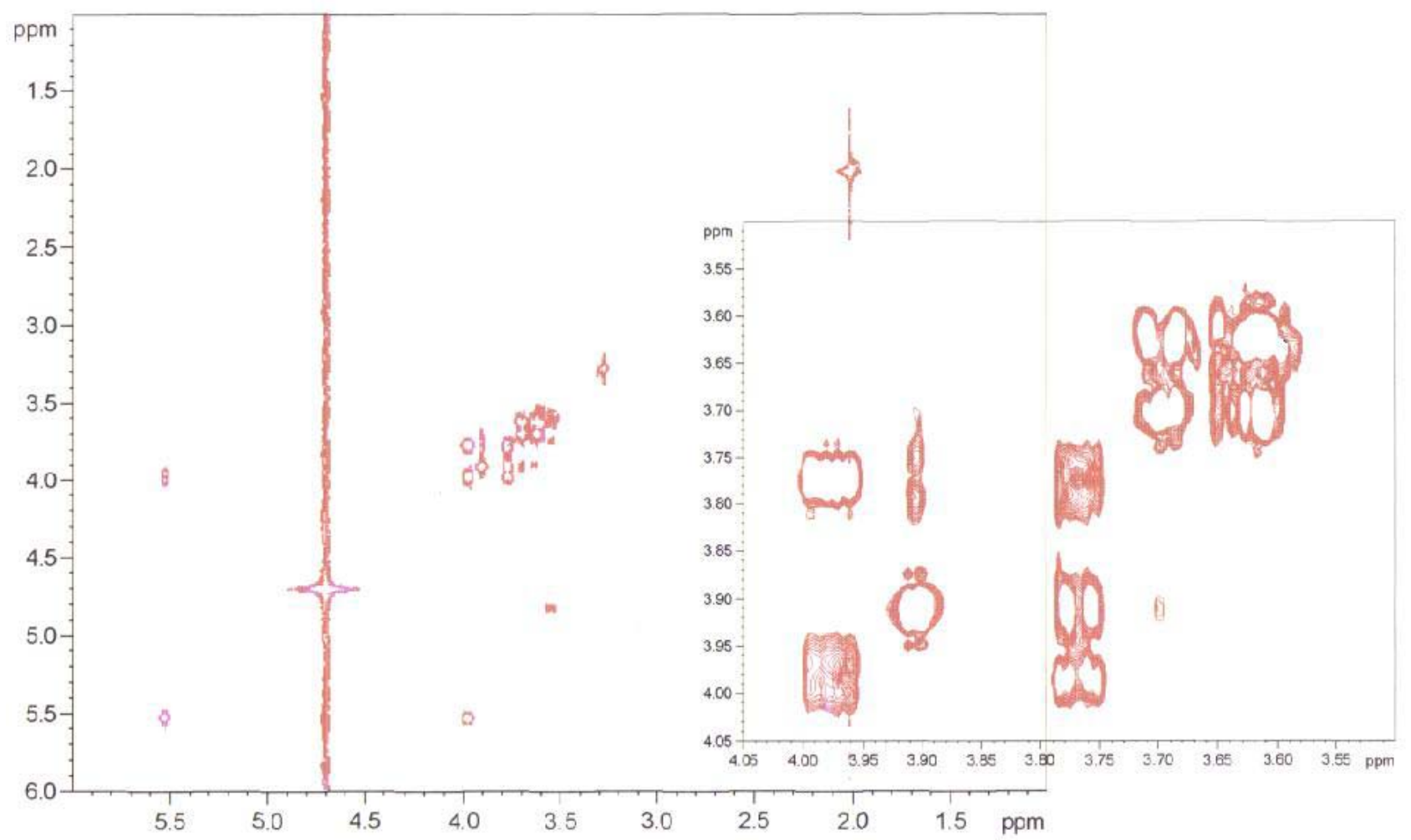


$N$-Pentanoyl- $\alpha$-D-glucopyranosylamine (19b)

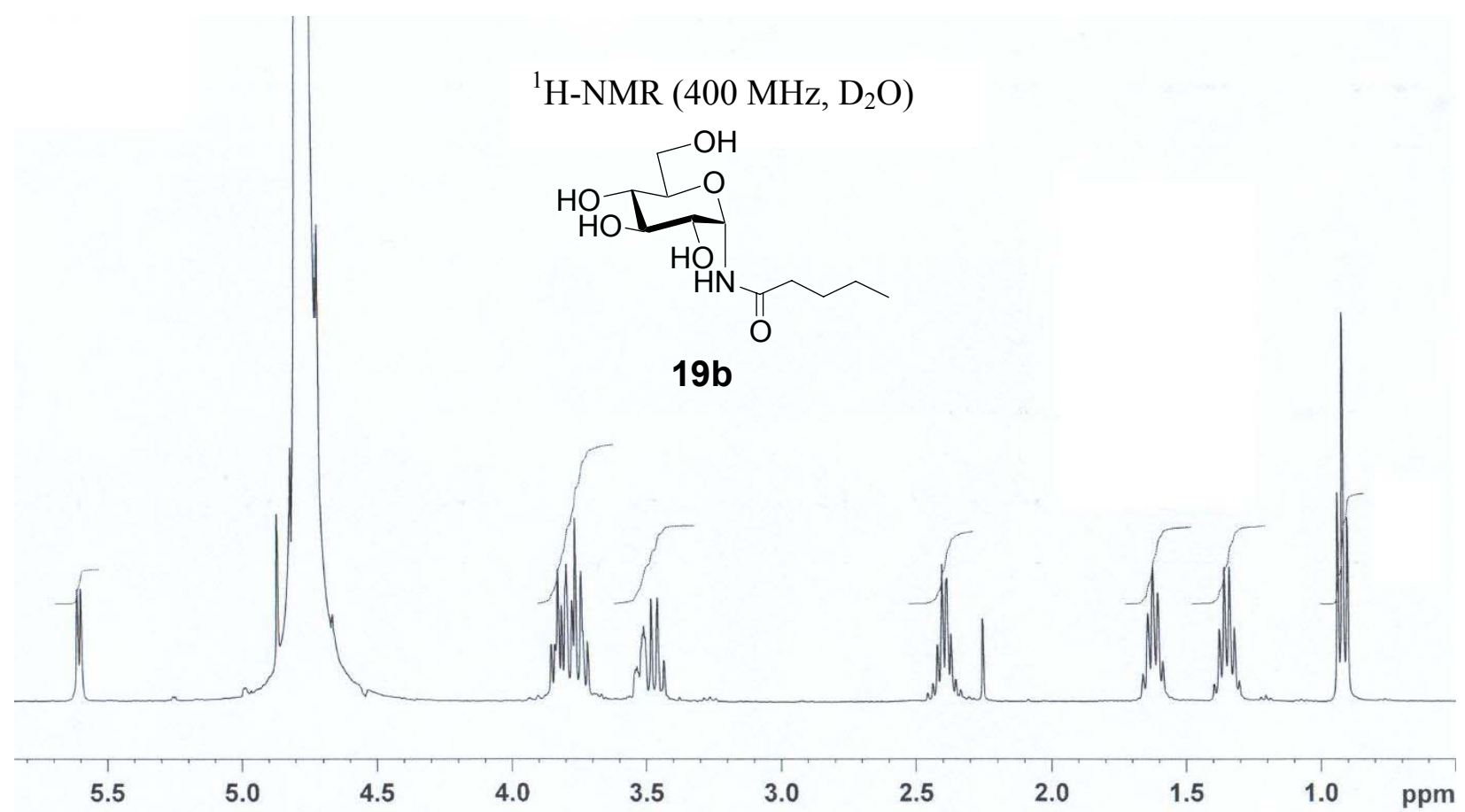

${ }^{13} \mathrm{C}-\mathrm{NMR}\left(100.6 \mathrm{MHz}, \mathrm{D}_{2} \mathrm{O}\right)$

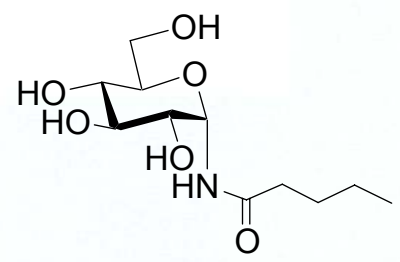

$19 b$

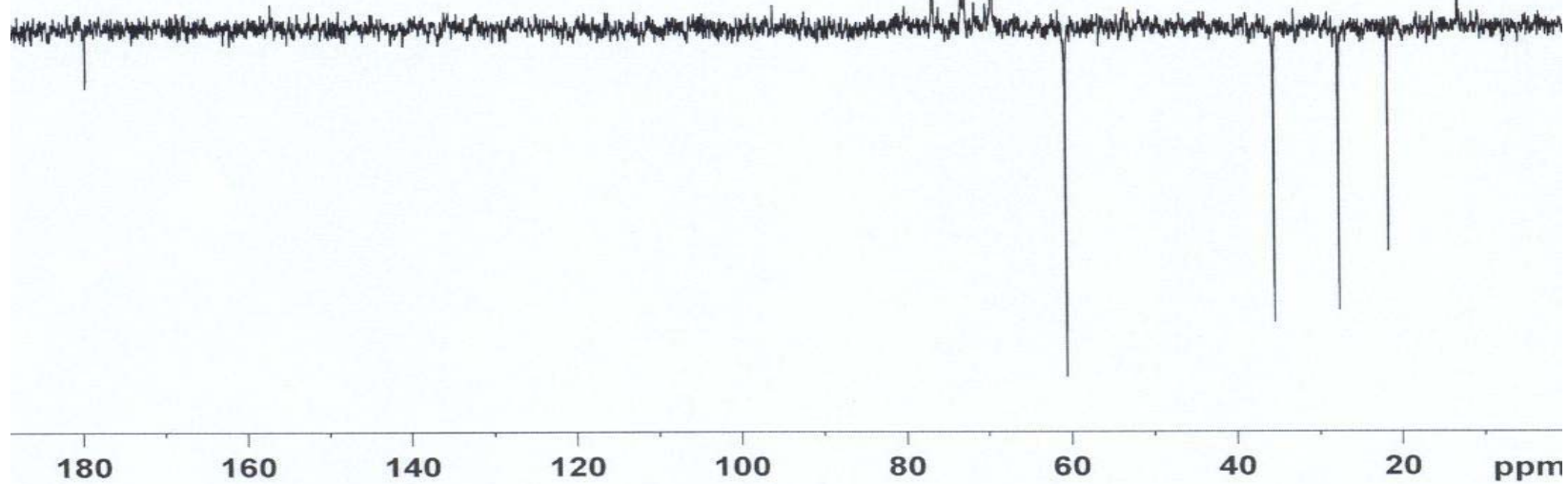




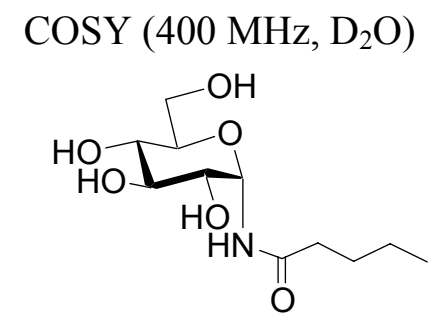

19b

ppm

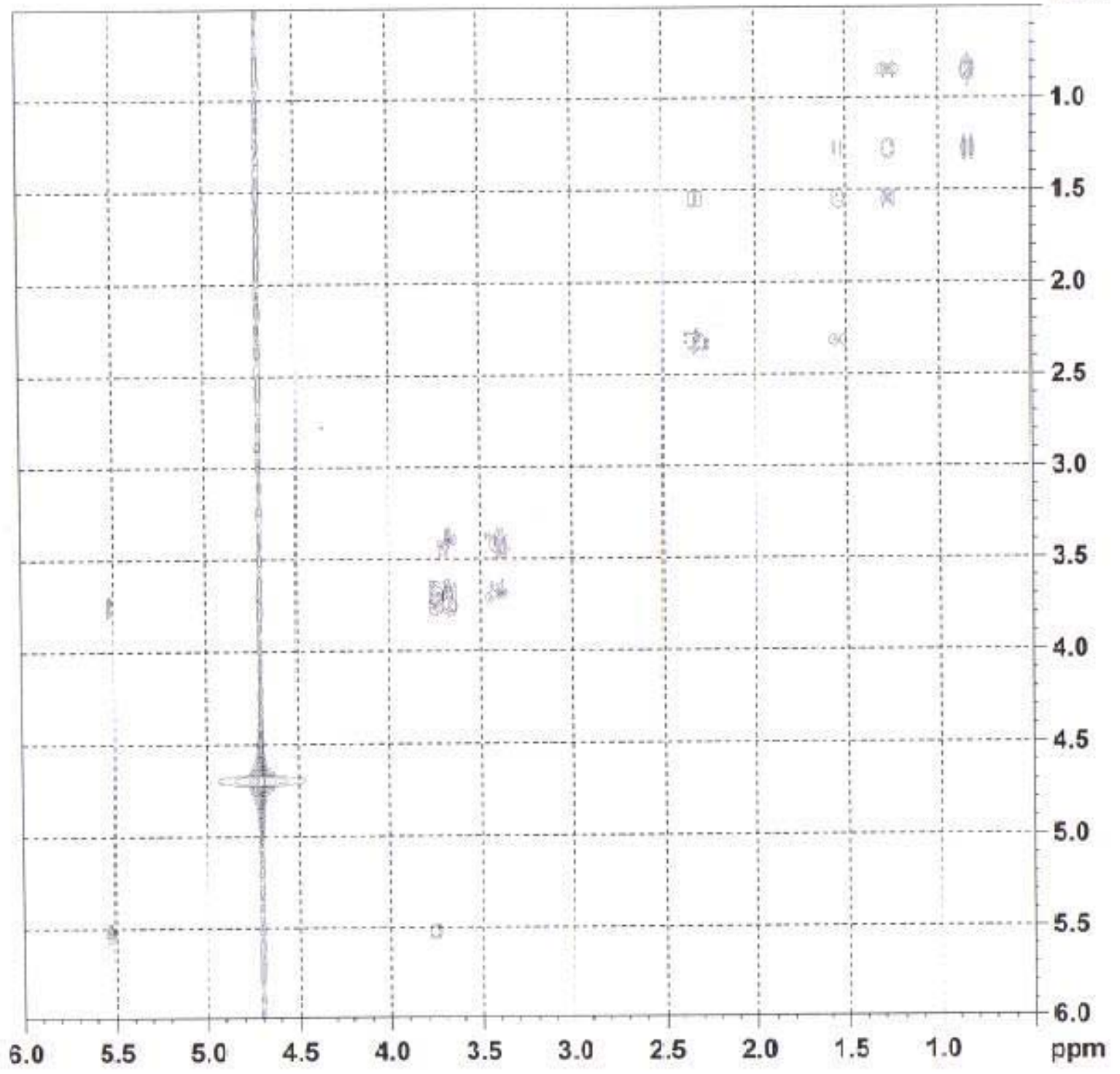




\section{$N^{\gamma}$-( $\alpha$-D-glucopyranosyl)-L-asparagine- $O$-methyl ester $(19 \mathrm{~g})$}

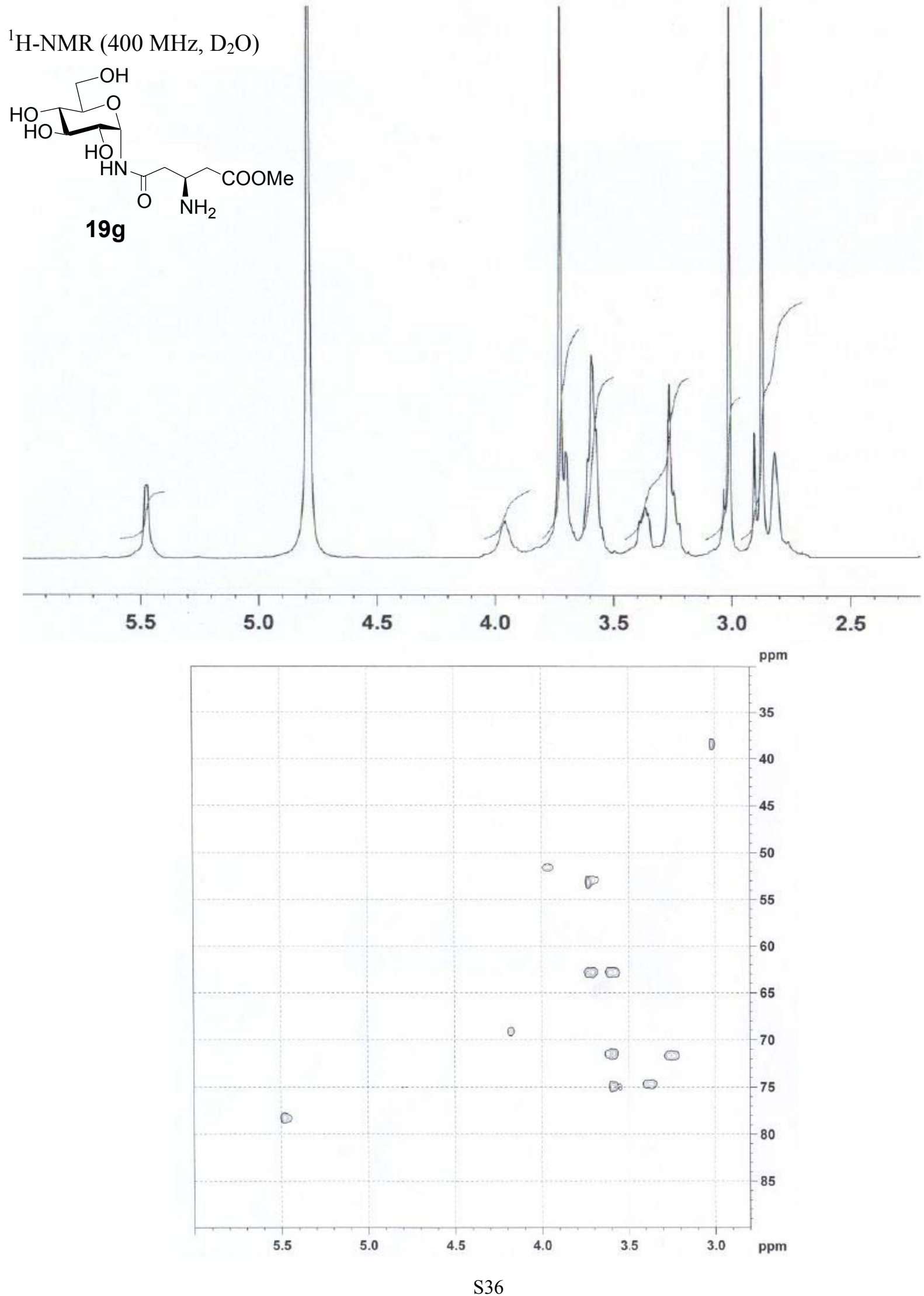




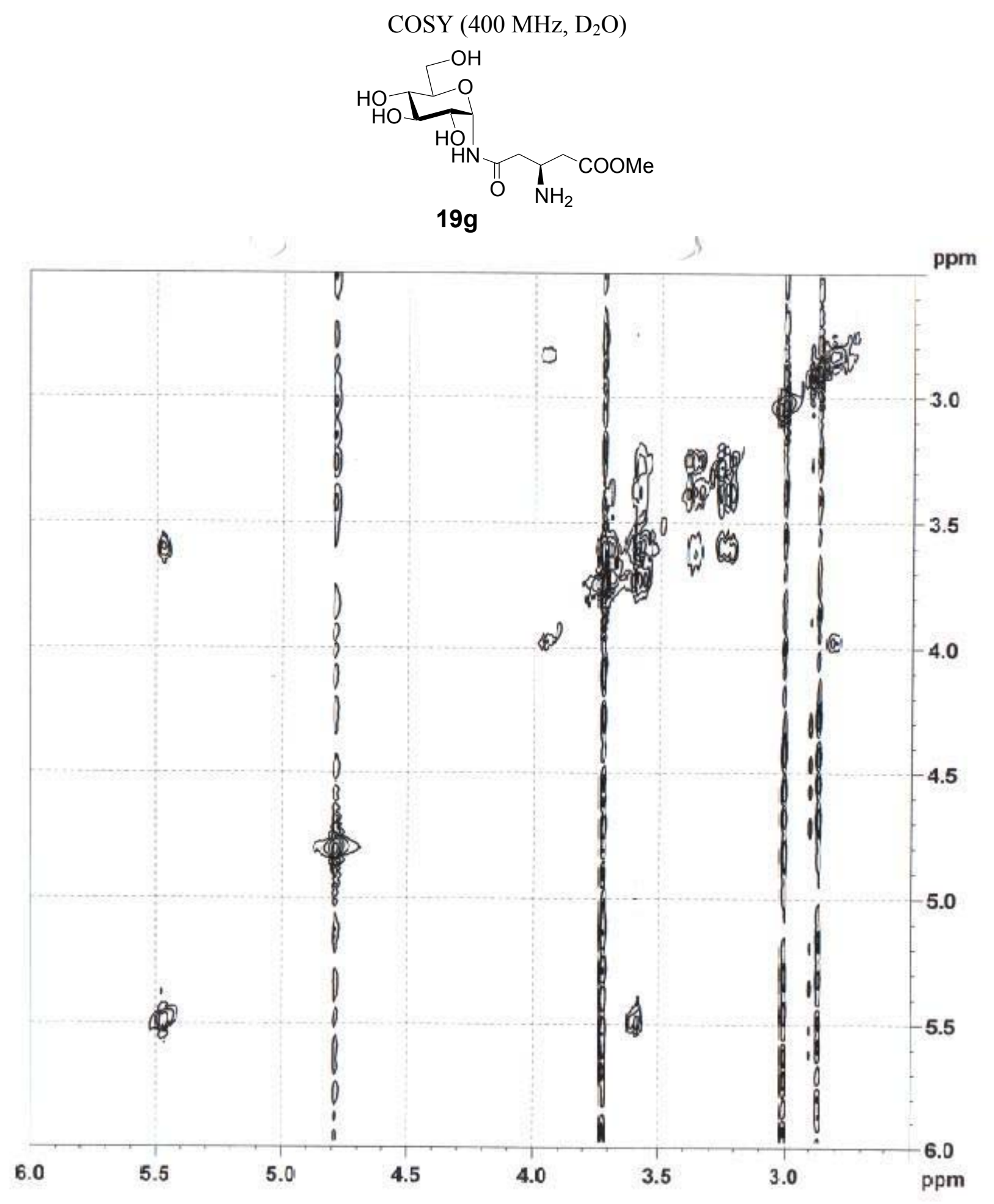


$N^{\alpha}$-t-butoxycarbonyl- $N^{\gamma}$-( $\alpha$-D-glucopyranosyl)-L-asparagine (19h)

${ }^{1} \mathrm{H}-\mathrm{NMR}\left(400 \mathrm{MHz}, \mathrm{D}_{2} \mathrm{O}\right)$



$\prod_{\mathrm{NHBoc}}^{\mathrm{COOH}}$

$19 \mathrm{~h}$
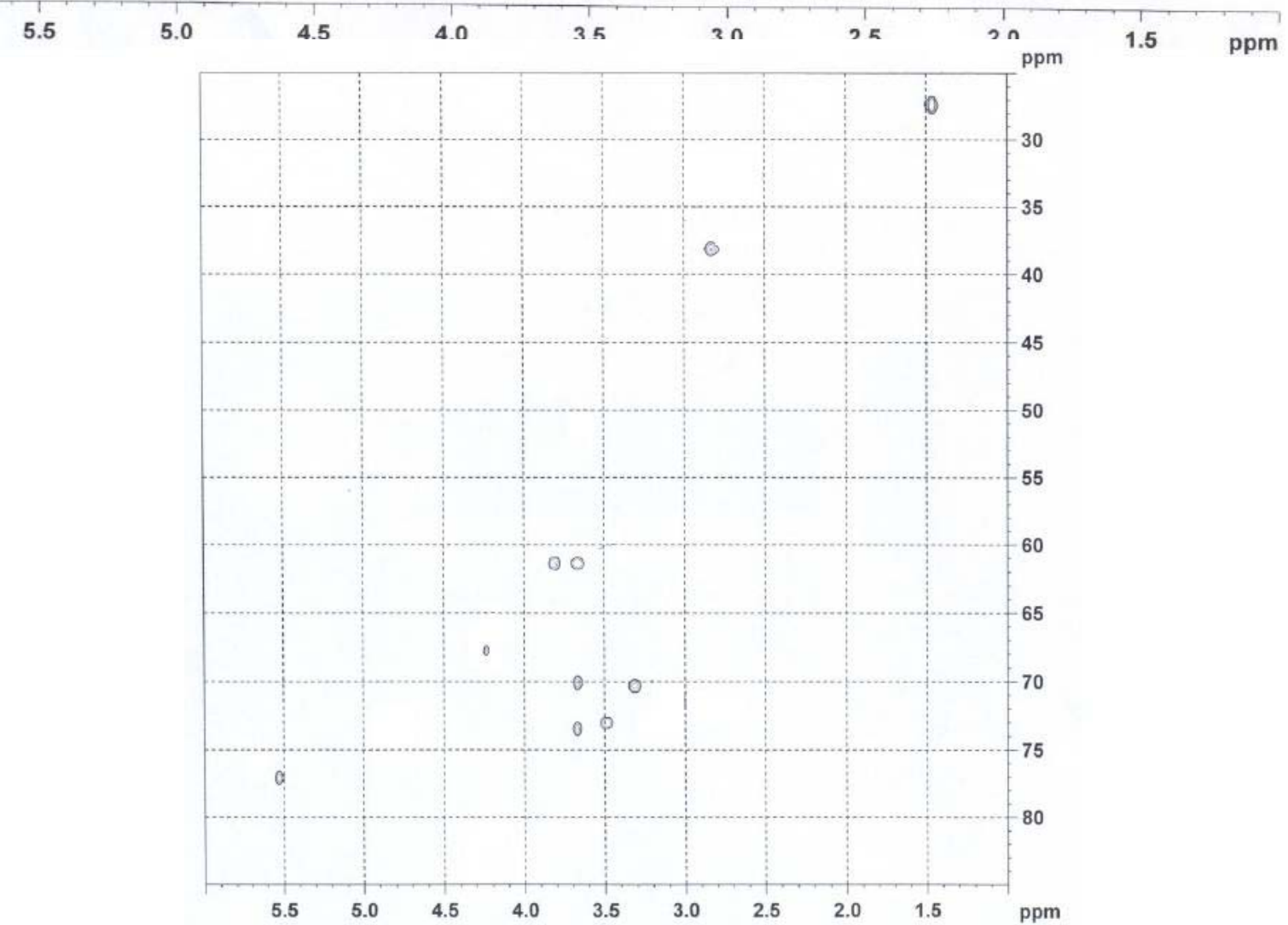


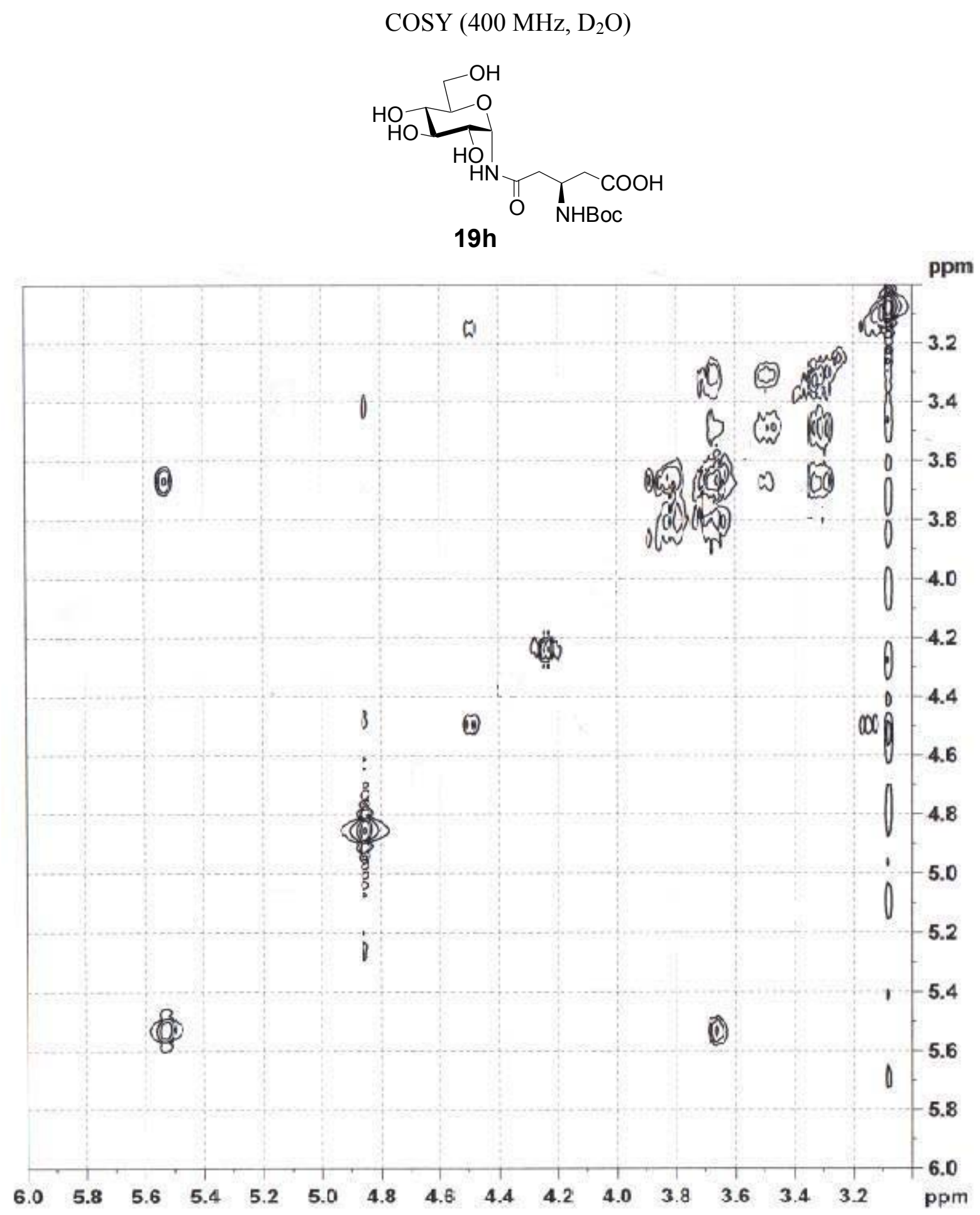


$N^{\alpha}$-acetyl- $N^{\gamma}-(2,3,4,6$-tetra- $O$-acetyl- $\alpha$-D-glucopyranosyl)-L-asparagine- $O$-methyl ester (21g)

${ }^{1} \mathrm{H}-\mathrm{NMR}\left(400 \mathrm{MHz}, \mathrm{CDCl}_{3}\right)$

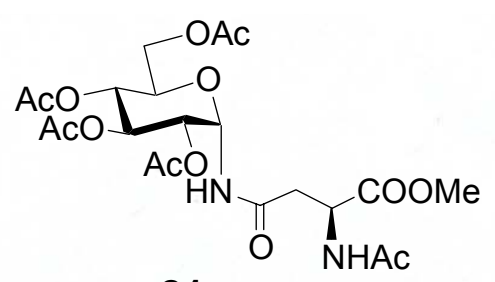

21g

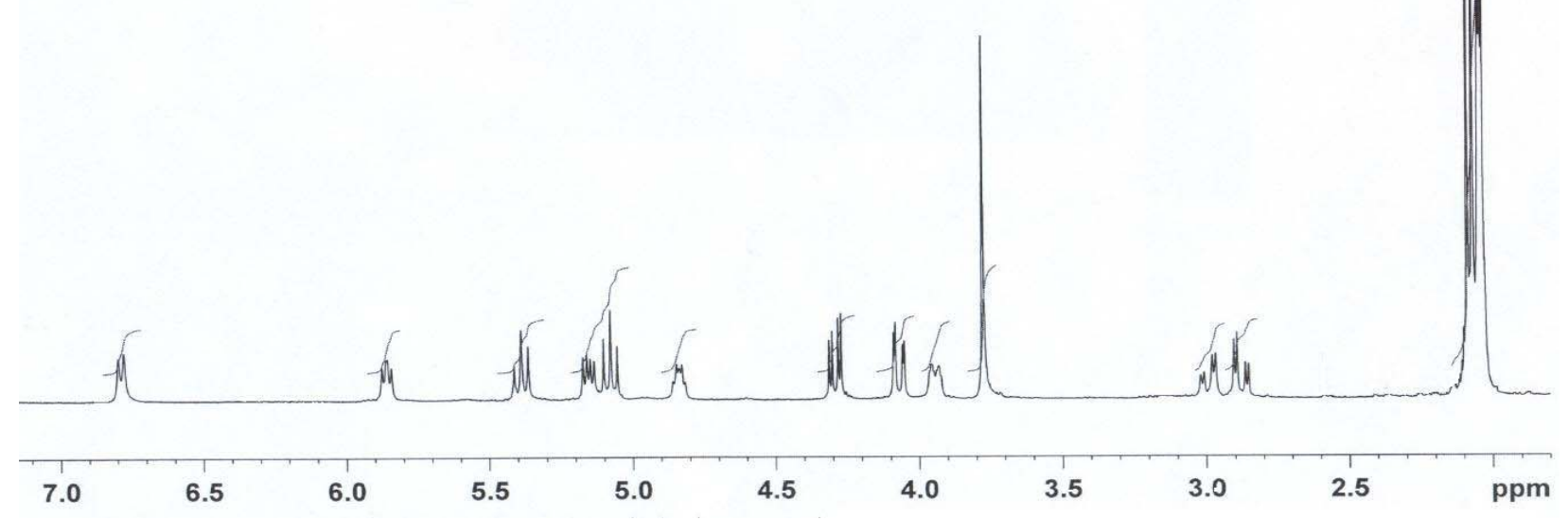

${ }^{13} \mathrm{C}-\mathrm{NMR}\left(100.6 \mathrm{MHz}, \mathrm{CDCl}_{3}\right)$

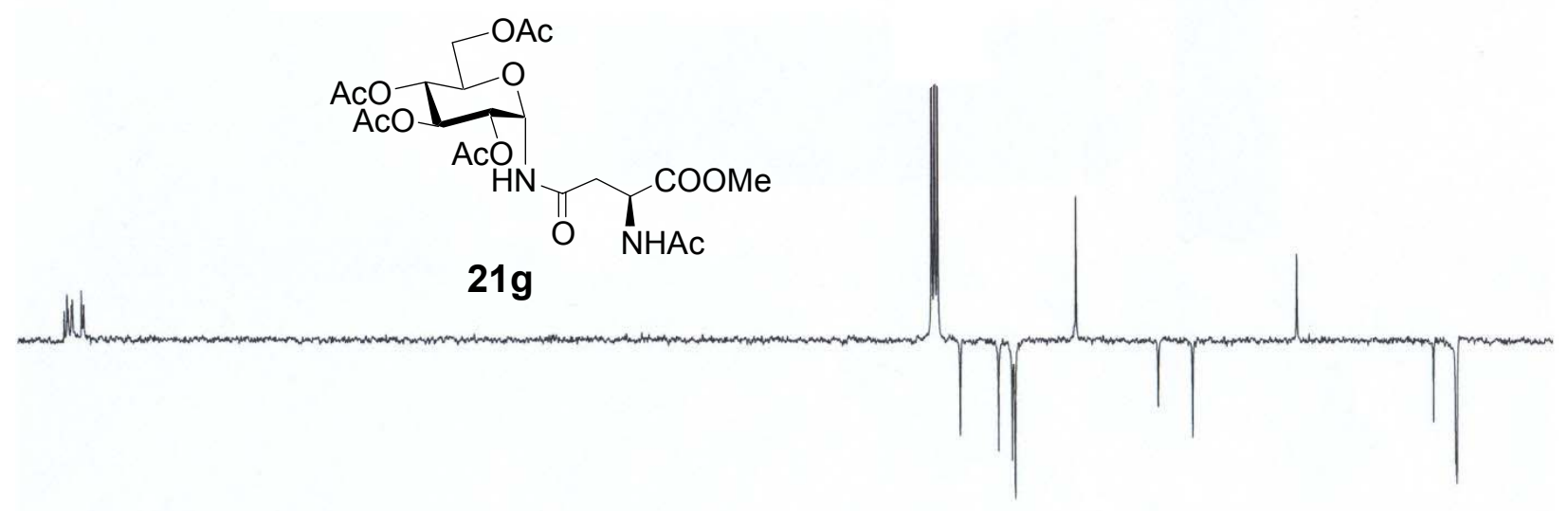

$\begin{array}{lllllllllllllllll}170 & 160 & 150 & 140 & 130 & 120 & 110 & 100 & 90 & 80 & 70 & 60 & 50 & 40 & 30 & 20 & \mathrm{ppm}\end{array}$


$\operatorname{COSY}\left(400 \mathrm{MHz}, \mathrm{CDCl}_{3}\right)$

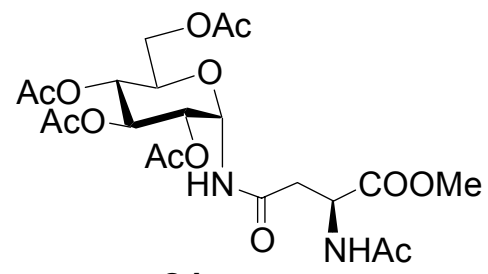

21g

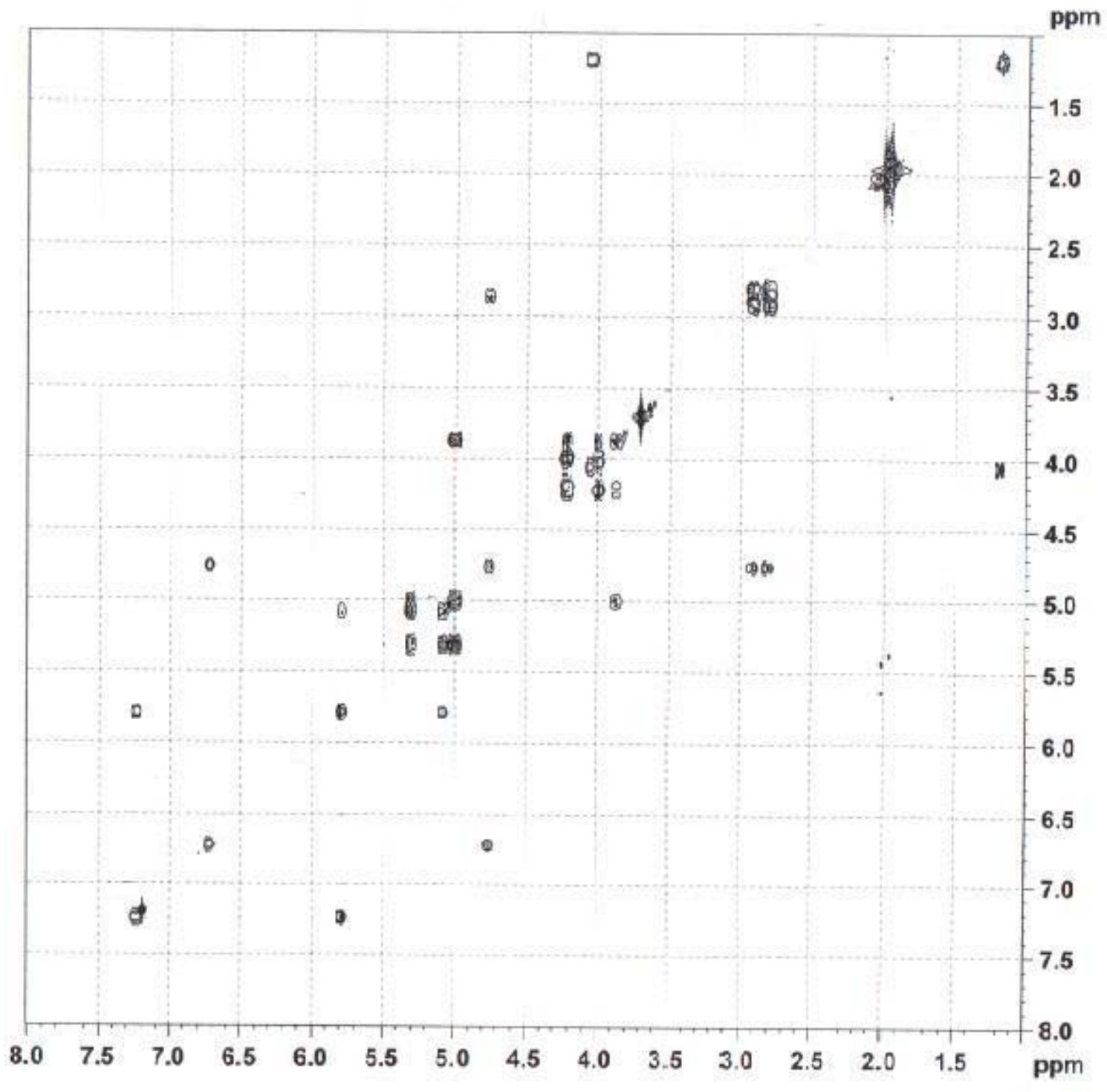


$N^{\alpha}$-carbobenzyloxy- $N^{\gamma}$-(2,3,4,6-tetra- $O$-acetyl- $\beta$-D-glucopyranosyl)-L-asparagine- $O$-methyl ester (13g)

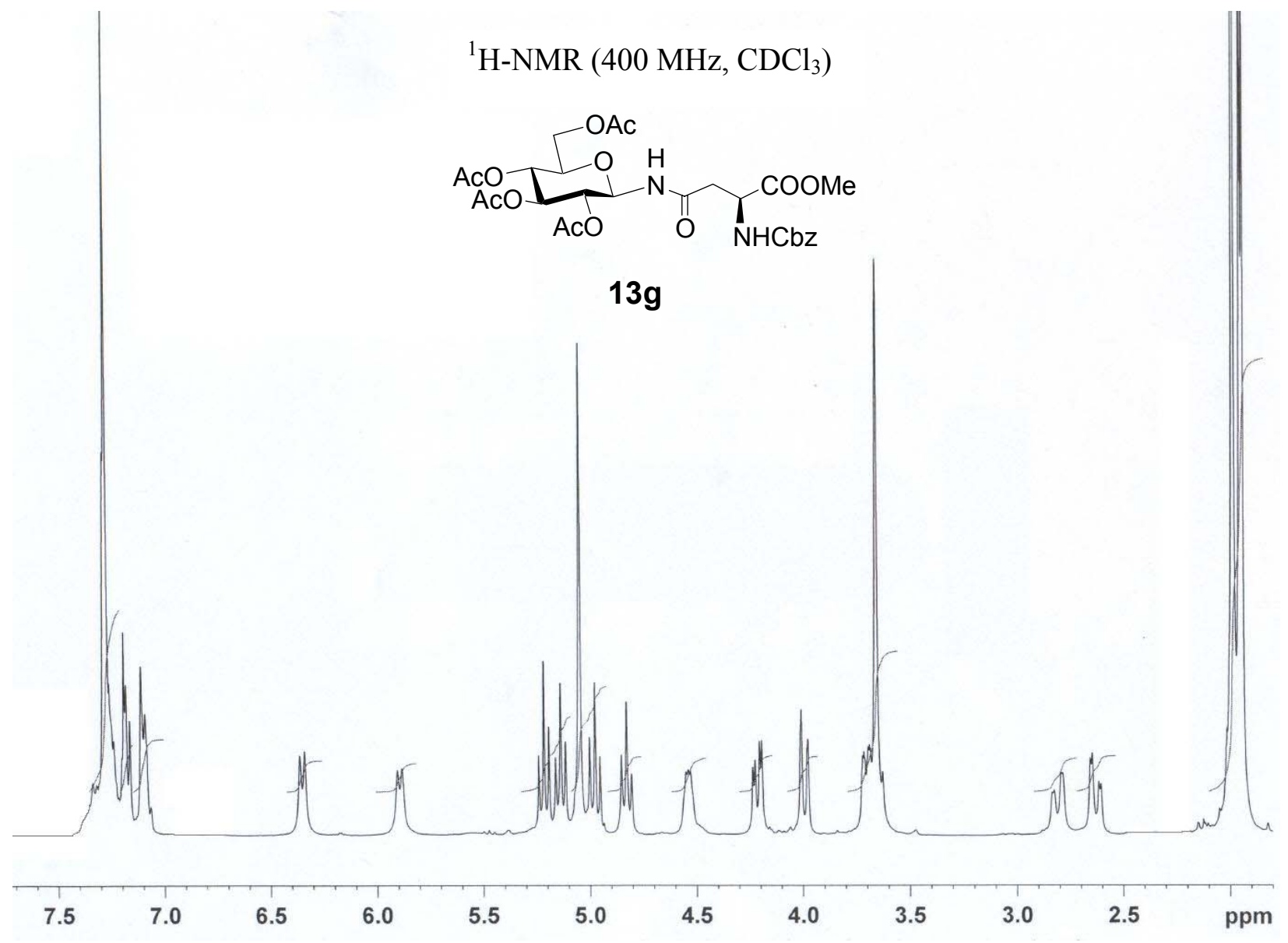

${ }^{13} \mathrm{C}-\mathrm{NMR}\left(100.6 \mathrm{MHz}, \mathrm{CDCl}_{3}\right)$

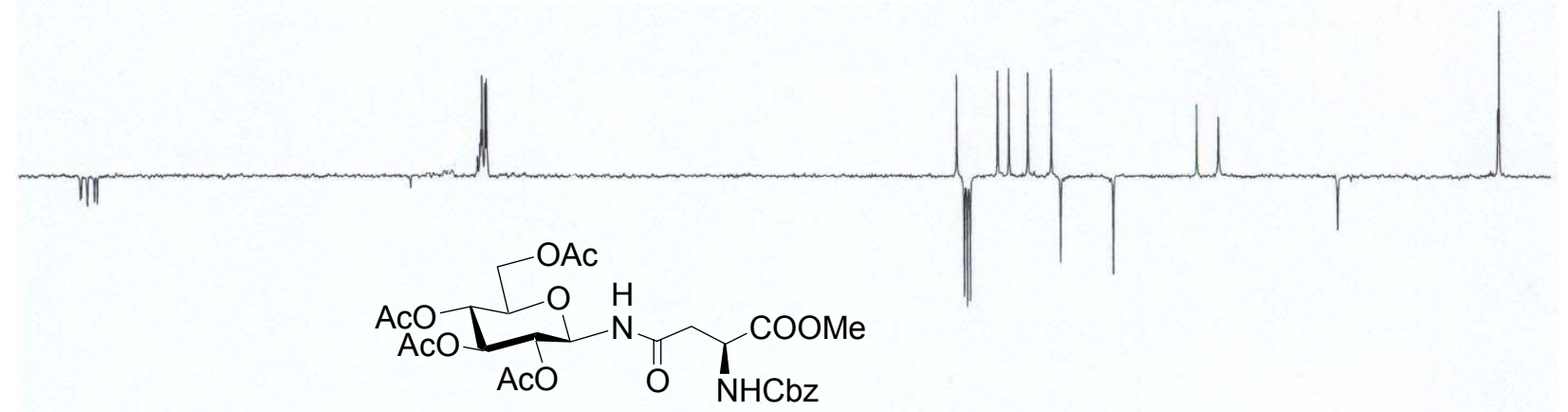

$13 g$

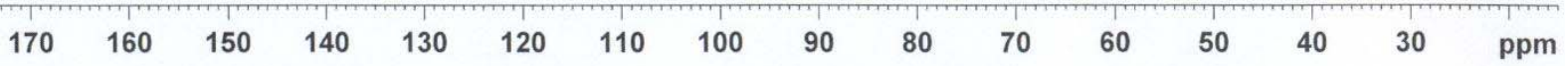


COSY $\left(400 \mathrm{MHz}, \mathrm{CDCl}_{3}\right)$

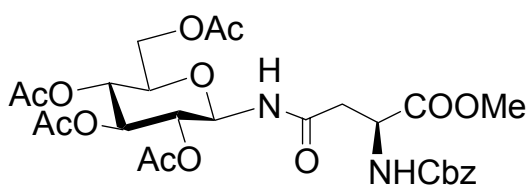

$13 g$

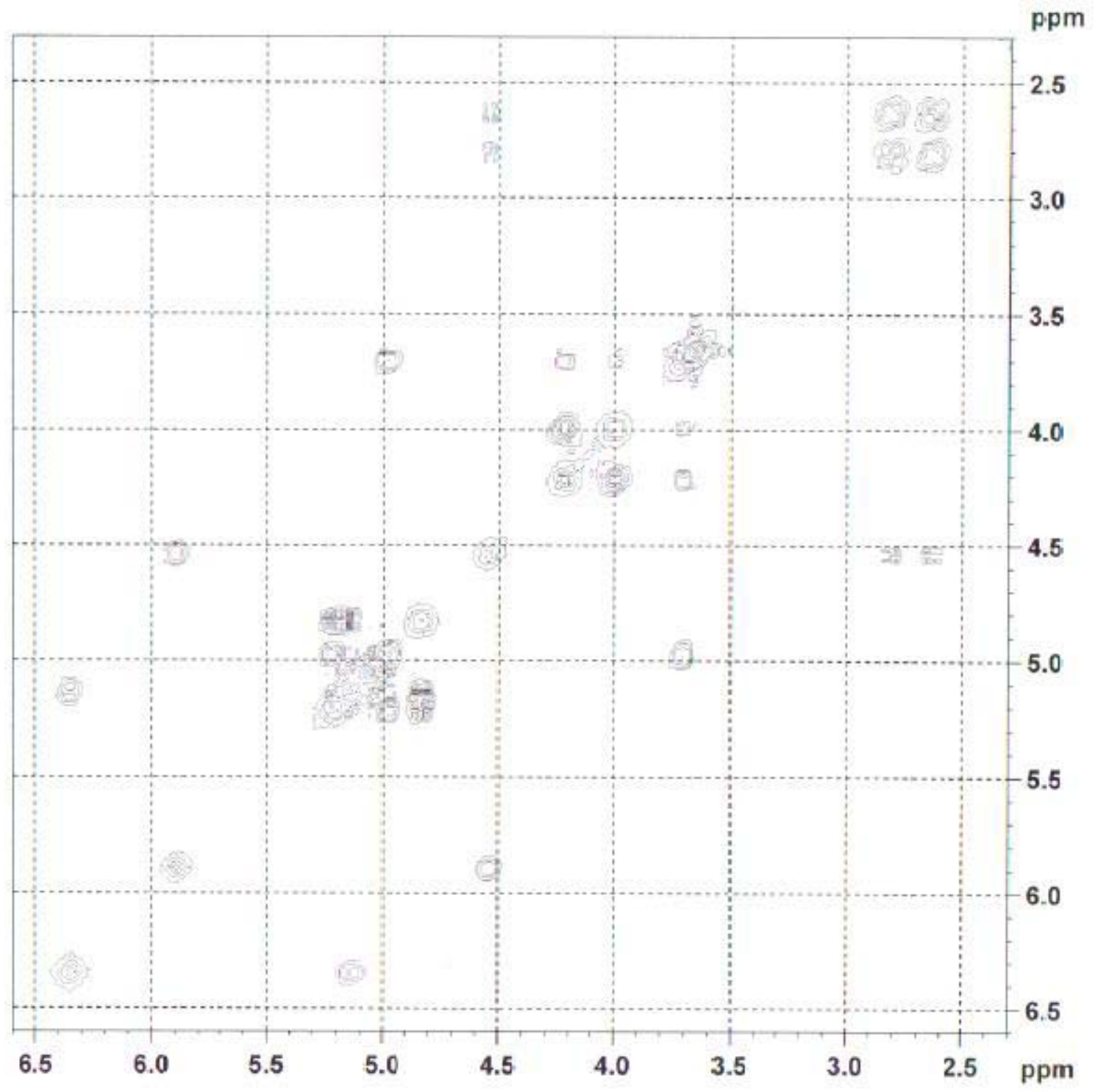


$N^{\alpha}$-carbonzyloxy- $N^{\gamma}$-(3,4,6-tri- $O$-acetyl-2- $N$-acetyl-2-deoxy- $\beta$-D-glucopyranosyl)-L-asparagine- $O$ methyl ester (14g)

${ }^{1} \mathrm{H}-\mathrm{NMR}\left(400 \mathrm{MHz}, \mathrm{CDCl}_{3}\right)$

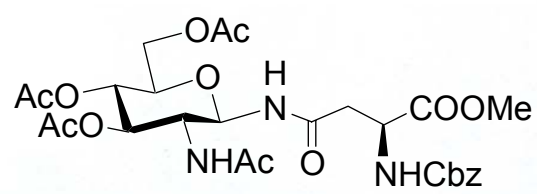

$14 \mathrm{~g}$

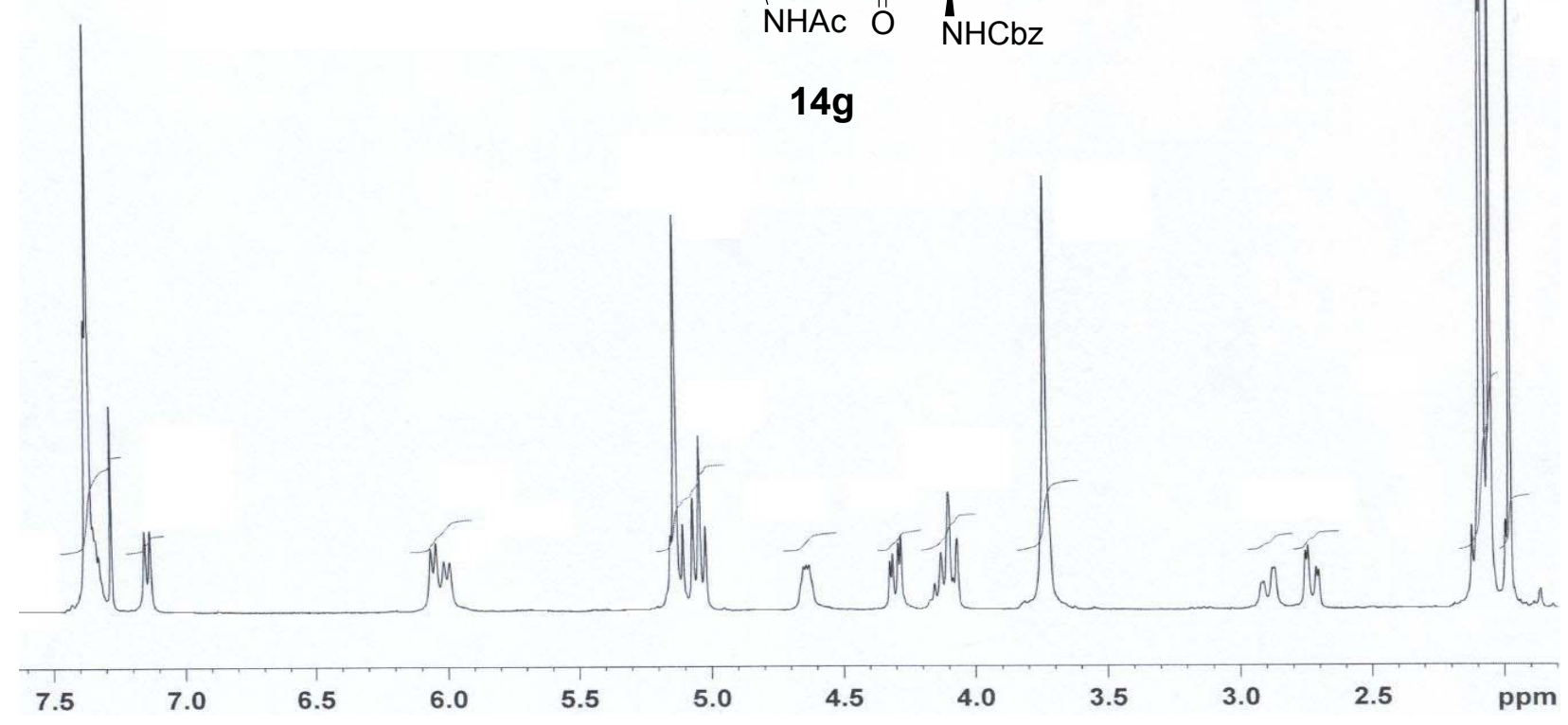

${ }^{13} \mathrm{C}-\mathrm{NMR}\left(100.6 \mathrm{MHz}, \mathrm{CDCl}_{3}\right)$

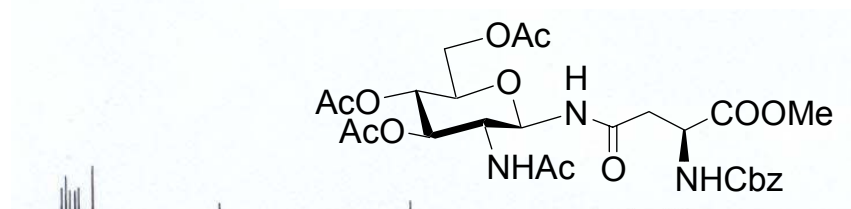

$\begin{array}{lllllllllllllllllllll}170 & 160 & 150 & 140 & 130 & 120 & 110 & 100 & 90 & 80 & 70 & 60 & 50 & 40 & 30 & 20 & \mathrm{ppm}\end{array}$


COSY $\left(400 \mathrm{MHz}, \mathrm{CDCl}_{3}\right)$

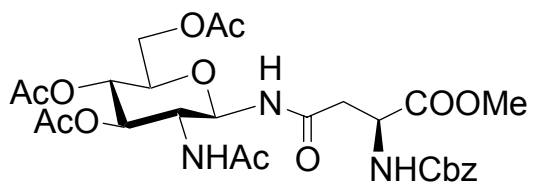

$14 \mathrm{~g}$

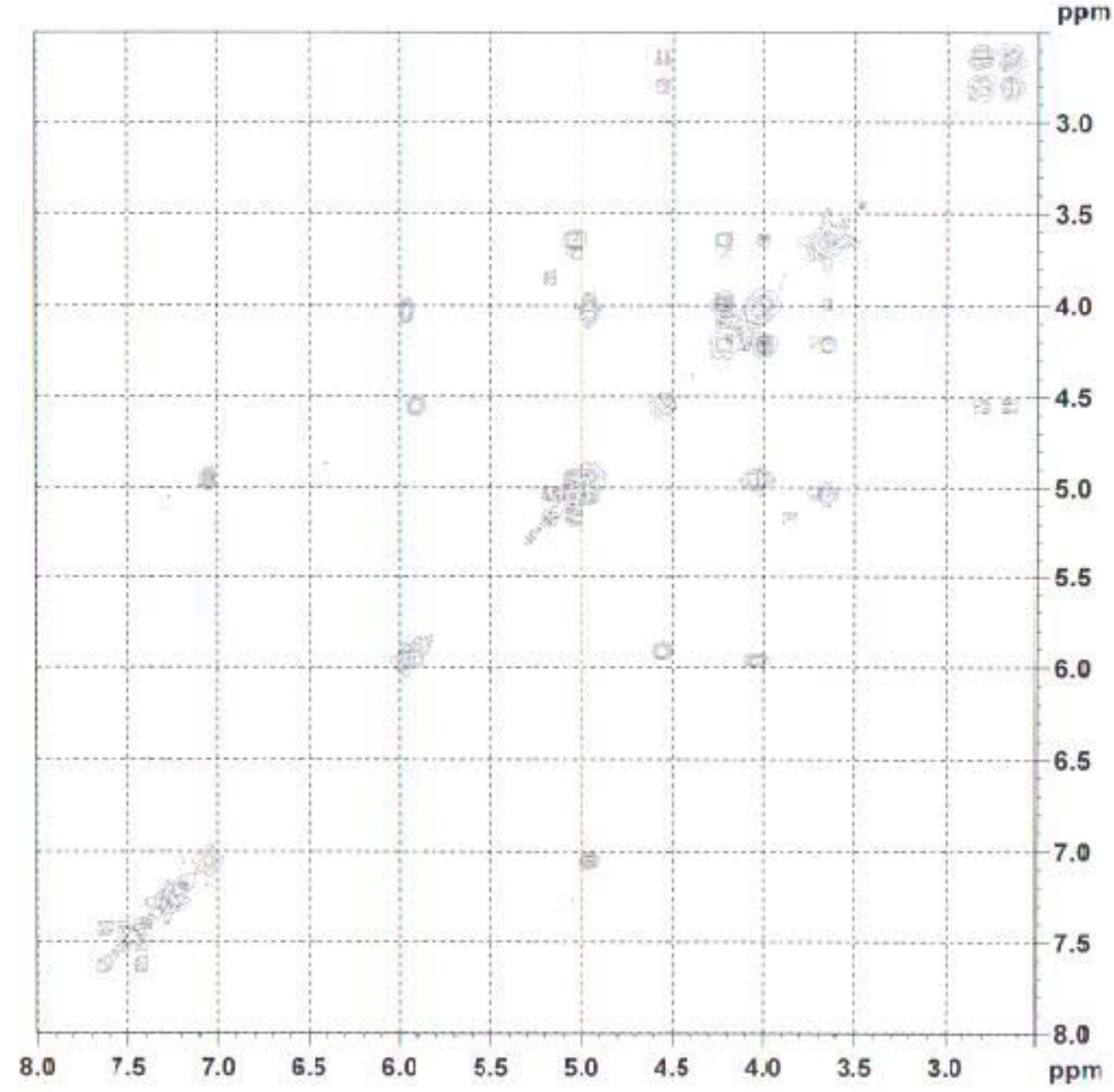


1- $N$ - Pentanedioic acid -2- $N$-phthalimido-2-deoxy-3,4,6-tri- $O$-acetyl- $\beta$-D-glucopyranosyl amide methyl ester (16f)

${ }^{1} \mathrm{H}-\mathrm{NMR}\left(400 \mathrm{MHz}, \mathrm{CDCl}_{3}\right)$

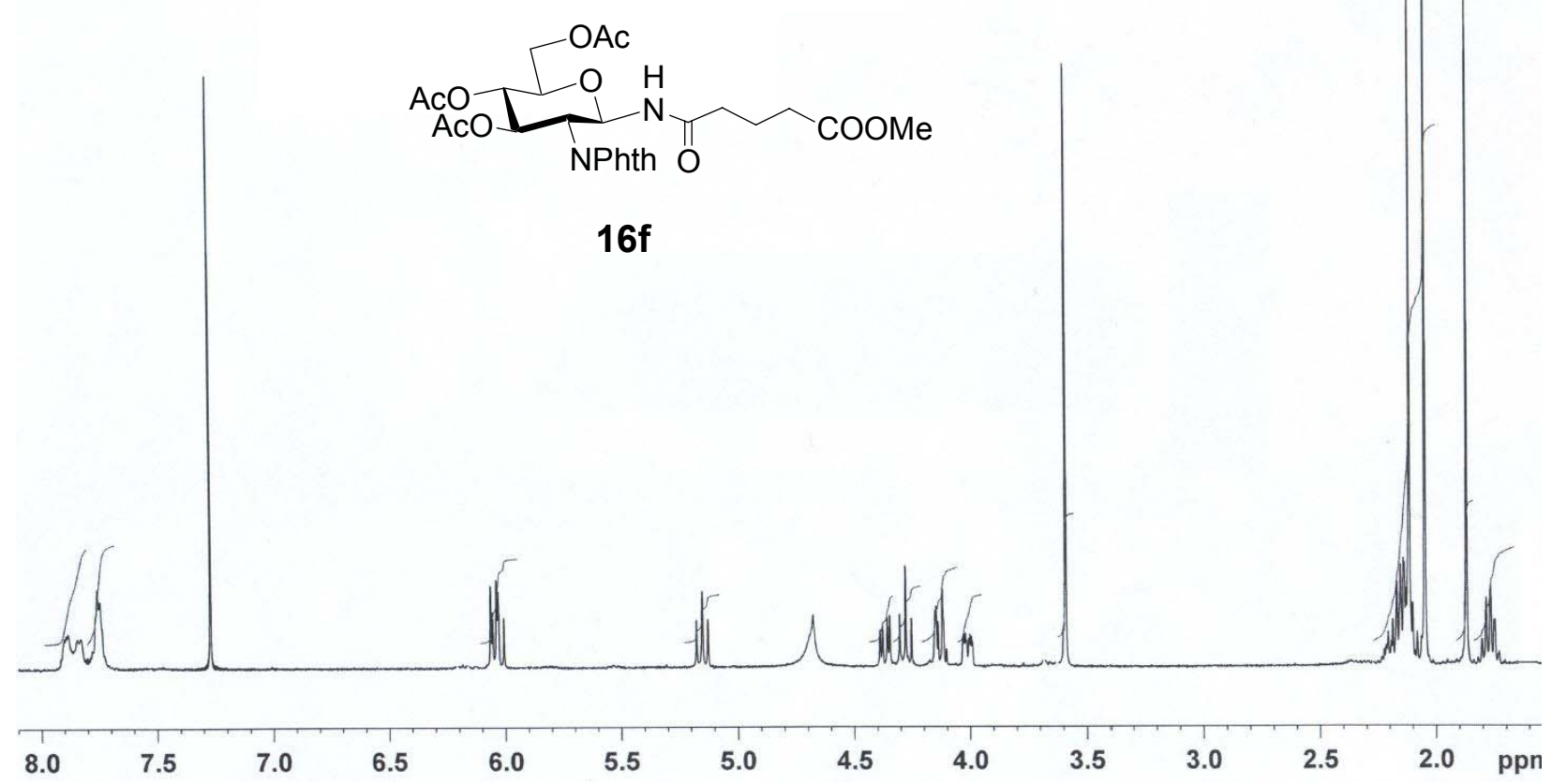

${ }^{13} \mathrm{C}-\mathrm{NMR}\left(100.6 \mathrm{MHz}, \mathrm{CDCl}_{3}\right)$
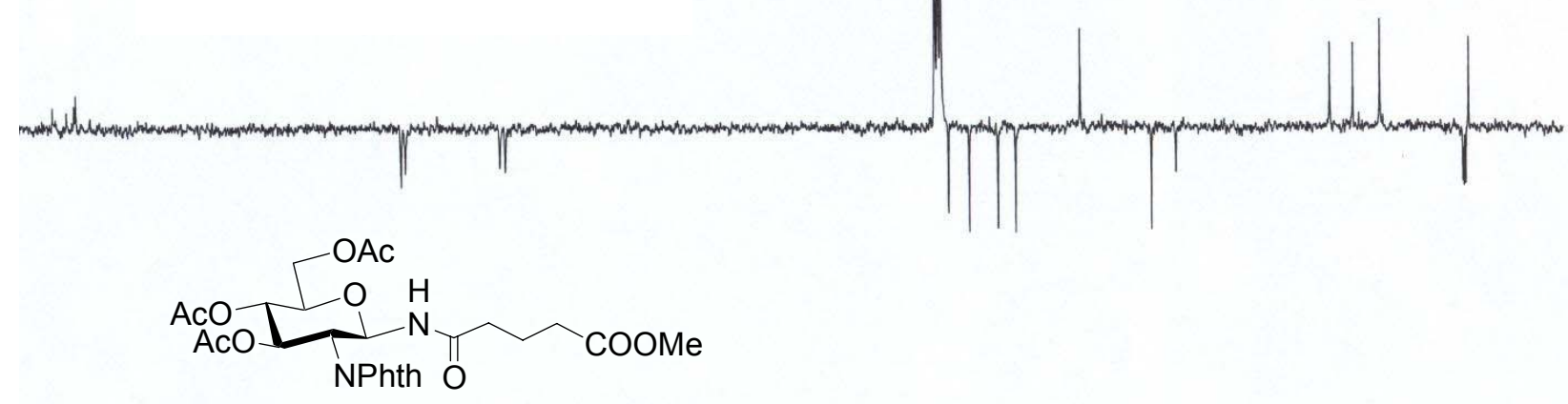

$16 f$

$\begin{array}{lllllllllllllllll}170 & 160 & 150 & 140 & 130 & 120 & 110 & 100 & 90 & 80 & 70 & 60 & 50 & 40 & 30 & 20 & \mathrm{ppm}\end{array}$


COSY $\left(400 \mathrm{MHz}, \mathrm{CDCl}_{3}\right)$

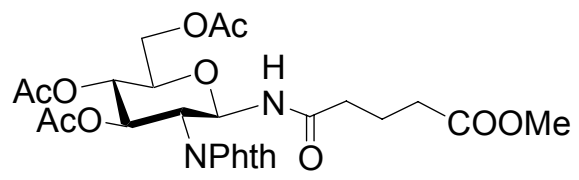

$16 f$

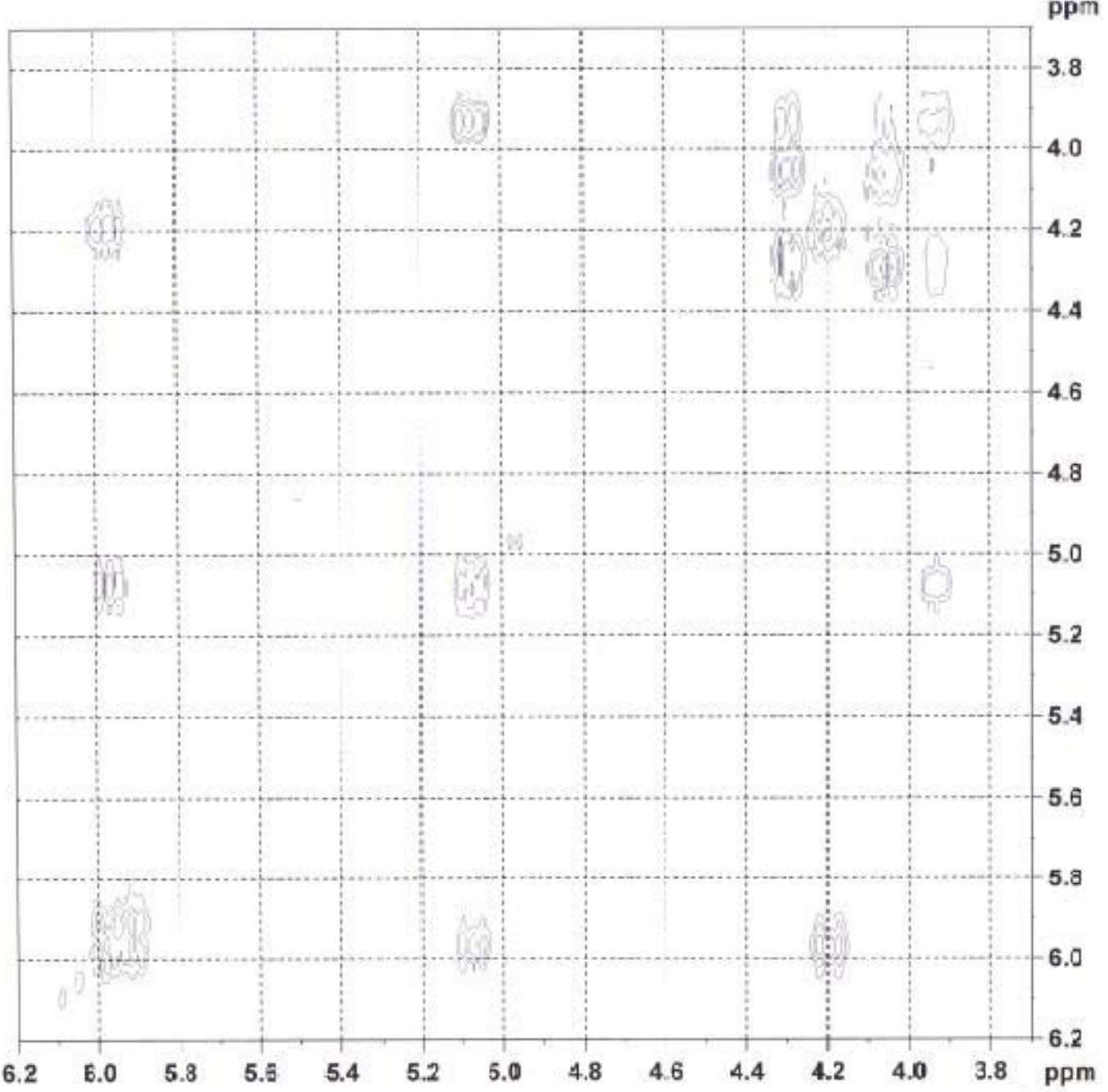

